\title{
GENERALIZED JOHNSON HOMOMORPHISMS FOR EXTENDED N-SERIES
}

\author{
KAZUO HABIRO AND GWÉNAËL MASSUYEAU
}

\begin{abstract}
The Johnson filtration of the mapping class group of a compact, oriented surface is the descending series consisting of the kernels of the actions on the nilpotent quotients of the fundamental group of the surface. Each term of the Johnson filtration admits a Johnson homomorphism, whose kernel is the next term in the filtration. In this paper, we consider a general situation where a group acts on a group with a filtration called an extended $N$-series. We develop a theory of Johnson homomorphisms in this general setting, including many known variants of the original Johnson homomorphisms as well as several new variants.
\end{abstract}

\section{INTRODUCTION}

In the late seventies and eighties, Johnson studied the algebraic structure of the mapping class group of a compact, oriented surface $\Sigma$ by examining its action on the lower central series of $\pi_{1}(\Sigma)$ [13]. He introduced a filtration of the mapping class group, which is now called the Johnson filtration, and defined homomorphisms on the terms of this filtration, called the Johnson homomorphisms. His study was preceded by Andreadakis' work on the automorphism group of a free group [1], and further developed by Morita [24]. So far, there have been several studies on variants of the Johnson filtrations and homomorphisms for mapping class groups and other groups, including the works [2, 3, 6, 14, 17, 18, 20, 23, 26, 28, 31, 32], where the lower central series are replaced with some other descending series.

The purpose of this paper is to generalize the Johnson filtrations and homomorphisms to an arbitrary group acting on another group with a descending series called an extended $\mathrm{N}$-series. Our constructions do not only give a generalized setting in order to view the above-mentioned variants from a unified viewpoint, but also provide new variants of the Johnson filtration and homomorphisms for the mapping class group of a handlebody.

1.1. Extended $\mathbf{N}$-series and extended graded Lie algebras. An $\mathrm{N}$-series $K_{+}=\left(K_{i}\right)_{i \geq 1}$ of a group $K$, introduced by Lazard [16], is a descending series

$$
K=K_{1} \geq K_{2} \geq \cdots
$$

such that $\left[K_{i}, K_{j}\right] \leq K_{i+j}$ for all $i, j \geq 1$. The most familiar example of an N-series is the lower central series $\Gamma_{+} K=\left(\Gamma_{i} K\right)_{i \geq 1}$ defined inductively by $\Gamma_{1} K=K$ and $\Gamma_{i+1} K=\left[K, \Gamma_{i} K\right]$ for $i \geq 1$. It is the smallest $\mathrm{N}$-series of $K$, i.e., we have $\Gamma_{i} K \leq K_{i}$ for all $i \geq 1$ and for all $\mathrm{N}$-series $\left(K_{i}\right)_{i \geq 1}$ of $K$.

Date: September 23, 2017 (First version: July 24, 2017). 
By a graded Lie algebra we mean a Lie algebra $L_{+}=\bigoplus_{i \geq 1} L_{i}$ over $\mathbb{Z}$ such that $\left[L_{i}, L_{j}\right] \subset L_{i+j}$ for $i, j \geq 1$. To every N-series $K_{+}$is associated a graded Lie algebra

$$
\mathrm{gr}_{+}\left(K_{+}\right)=\bigoplus_{i \geq 1} K_{i} / K_{i+1}
$$

where the Lie bracket is induced by the commutator operation.

An extended $N$-series, studied in this paper, is a natural generalization of $\mathrm{N}$ series. An extended N-series $K_{*}=\left(K_{i}\right)_{i \geq 0}$ of a group $K$ is a descending series

$$
K=K_{0} \geq K_{1} \geq K_{2} \geq \cdots
$$

such that $\left[K_{i}, K_{j}\right] \leq K_{i+j}$ for all $i, j \geq 0$. Alternatively, a descending series (1.1) is an extended N-series if the positive part $K_{+}=\left(K_{i}\right)_{i \geq 1}$ is an N-series and if $K_{i}$ is a normal subgroup of $K$ for all $i \geq 1$. Note that an N-series $K_{+}$canonically extends to an extended N-series by setting $K_{0}=K_{1}$.

An extended graded Lie algebra (abbreviated as eg-Lie algebra) $L_{\bullet}=\left(L_{i}\right)_{i \geq 0}$ is a pair of a graded Lie algebra $L_{+}=\bigoplus_{i>1} L_{i}$ and a group $L_{0}$ acting on $L_{+}$. To each extended $\mathrm{N}$-series $K_{*}$, we associate an eg-Lie algebra gr $\operatorname{gr}_{\bullet}\left(K_{*}\right)=\left(\operatorname{gr}_{i}\left(K_{*}\right)\right)_{i \geq 0}$, consisting of the graded Lie algebra $\operatorname{gr}_{+}\left(K_{*}\right)=\mathrm{gr}_{+}\left(K_{+}\right)$associated to the Nseries part $K_{+}$of $K_{*}$, and the action of $\operatorname{gr}_{0}\left(K_{*}\right)=K_{0} / K_{1}$ on $\mathrm{gr}_{+}\left(K_{+}\right)$induced by conjugation.

1.2. Johnson filtrations and Johnson homomorphisms. To recall Johnson's approach to mapping class groups, assume that $\Sigma$ is a compact, connected, oriented surface with $\partial \Sigma \cong S^{1}$. Let $K=\pi_{1}(\Sigma, \star)$, where $\star \in \partial \Sigma$, and let $G$ be the mapping class group of $\Sigma$ relative to $\partial \Sigma$. The natural action of $G$ on $K$ gives rise to the Dehn-Nielsen representation

$$
\rho: G \longrightarrow \operatorname{Aut}(K) \text {. }
$$

Let $K_{+}=\Gamma_{+} K$ be the lower central series of $K$. The Johnson filtration $G_{*}=$ $\left(G_{m}\right)_{m \geq 0}$ of $G$ is defined by

$$
G_{m}=\operatorname{ker}\left(\rho_{m}: G \longrightarrow \operatorname{Aut}\left(K / K_{m+1}\right)\right) \text {, }
$$

where $\rho_{m}(g)\left(k K_{m+1}\right)=\rho(g)(k) K_{m+1}$. The series $G_{*}$ is an extended N-series. The subgroup $G_{1}$ is known as the Torelli group of $\Sigma$, and it is well known that $\bigcap_{m \geq 0} G_{m}=\{1\}$.

For $m \geq 1$, the mth Johnson homomorphism

$$
\tau_{m}: G_{m} \longrightarrow \operatorname{Hom}\left(K_{1} / K_{2}, K_{m+1} / K_{m+2}\right)
$$

is defined by

$$
\tau_{m}(g)\left(k K_{2}\right)=g(k) k^{-1} K_{m+2} \quad \text { for } g \in G_{m}, k \in K_{1} .
$$

Thus, $\tau_{m}$ measures the extent to which the action of $G_{m}$ on $K / K_{m+2}$ fails to be trivial; in particular, $\operatorname{ker}\left(\tau_{m}\right)=G_{m+1}$. We can identify $\operatorname{Hom}\left(K_{1} / K_{2}, K_{m+1} / K_{m+2}\right)$ with the group $\operatorname{Der}_{m}\left(\operatorname{gr}_{+}(K)\right)$ of degree $m$ derivations of $\mathrm{gr}_{+}(K)$, since the associated graded Lie algebra $\operatorname{gr}_{+}(K)=\bigoplus_{m>1} K_{m} / K_{m+1}$ is free on its degree 1 part $K_{1} / K_{2}$. Thus the $\tau_{m}$ 's for $m \geq 1$ induce homomorphisms

$$
\bar{\tau}_{m}: G_{m} / G_{m+1} \longrightarrow \operatorname{Der}_{m}\left(\mathrm{gr}_{+}(K)\right),
$$

forming an injective morphism of graded Lie algebras

$$
\bar{\tau}_{+}: \mathrm{gr}_{+}(G) \longrightarrow \operatorname{Der}_{+}\left(\mathrm{gr}_{+}(K)\right),
$$


where $\operatorname{Der}_{+}\left(\operatorname{gr}_{+}(K)\right)=\bigoplus_{m \geq 1} \operatorname{Der}_{m}\left(\operatorname{gr}_{+}(K)\right)$ is the Lie algebra of positive-degree derivations of $\operatorname{gr}_{+}(K)$. This morphism of graded Lie algebras, which contains all the Johnson homomorphisms, was introduced by Morita [24, Theorem 4.8]; we call it the Johnson morphism. From an algebraic viewpoint, it is important to determine the image of $\bar{\tau}_{+}$, which is a Lie subalgebra of $\operatorname{Der}_{+}\left(\mathrm{gr}_{+}(K)\right)$. We refer the reader to Satoh's survey [36] for further details and references.

We can extend $\operatorname{Der}_{+}\left(\operatorname{gr}_{+}(K)\right)$ to an eg-Lie algebra Der $\left(\operatorname{gr}_{+}(K)\right)$, where the group $\operatorname{Der}_{0}\left(\operatorname{gr}_{+}(K)\right)=\operatorname{Aut}\left(\operatorname{gr}_{+}(K)\right)$ acts on $\operatorname{Der}_{+}\left(\operatorname{gr}_{+}(K)\right)$ by conjugation. Then the map $\bar{\tau}_{+}$naturally extends to a morphism of eg-Lie algebras

$$
\bar{\tau}_{\bullet}: \operatorname{gr}_{\bullet}(G) \longrightarrow \operatorname{Der}_{\bullet}\left(\operatorname{gr}_{+}(K)\right)
$$

whose degree 0 part

$$
\bar{\tau}_{0}: \operatorname{gr}_{0}(G)=G_{0} / G_{1} \longrightarrow \operatorname{Der}_{0}\left(\operatorname{gr}_{+}(K)\right) \simeq \operatorname{Aut}\left(H_{1}(\Sigma ; \mathbb{Z})\right)
$$

is given by the natural action of the mapping class group on homology.

1.3. The Johnson morphisms associated to extended $\mathbf{N}$-series actions. We develop a theory of Johnson homomorphisms in the general situation where an extended N-series $G_{*}=\left(G_{m}\right)_{m \geq 0}$ of a group $G$ acts on an extended N-series $K_{*}=\left(K_{m}\right)_{m \geq 0}$ of another group $K$. This means that a left action

$$
G \times K \longrightarrow K, \quad(g, k) \longmapsto g(k),
$$

of $G$ on $K$ satisfies

$$
g(k) k^{-1} \in K_{i+j} \quad \text { for all } g \in G_{i}, i \geq 0 \text { and } k \in K_{j}, j \geq 0 .
$$

We say that a group $G$ acts on an extended N-series $K_{*}$ if $g\left(K_{j}\right)=K_{j}$ for all $j \geq 0$. In this case, we have an extended N-series $\mathcal{F}_{*}^{K_{*}}(G)$ of $G$ acting on $K_{*}$, defined by

$$
\mathcal{F}_{i}^{K_{*}}(G)=\left\{g \in G \mid g(k) k^{-1} \in K_{i+j} \text { for all } k \in K_{j}, j \geq 0\right\} .
$$

We call $\mathcal{F}_{*}^{K_{*}}(G)$ the Johnson filtration of $G$ induced by $K_{*}$.

To each extended graded Lie algebra $L_{\bullet}$, we associate the derivation eg-Lie algebra Der. $\left(L_{\bullet}\right)$ (see Theorem 5.3). The degree 0 part $\operatorname{Der}_{0}\left(L_{\bullet}\right)$ is the automorphism group $\operatorname{Aut}\left(L_{\bullet}\right)$ of $L_{\bullet}$; the positive part $\operatorname{Der}_{+}\left(L_{\bullet}\right)$ is the Lie algebra of positivedegree derivations of $L_{\bullet}$. Here, for $m \geq 1$, a degree $m$ derivation of $L_{\bullet}$ consists of a degree $m$ derivation $d_{+}$of $L_{+}$and a 1-cocycle $d_{0}: L_{0} \rightarrow L_{m}$ satisfying certain compatibility condition (see Definition 5.1).

To each action of an extended N-series $G_{*}$ on an extended N-series $K_{*}$, we associate a morphism of extended graded Lie algebras

$$
\bar{\tau}_{\bullet}: \operatorname{gr}\left(G_{*}\right) \longrightarrow \operatorname{Der} \bullet\left(\operatorname{gr} \bullet\left(K_{*}\right)\right),
$$

which we call the Johnson morphism, and which generalizes (1.2). The morphism $\bar{\tau}_{\bullet}$ is injective if and only if $G_{*}$ is the Johnson filtration induced by $K_{*}$. (See Theorem 6.4.) 
1.4. The case of $\mathbf{N}$-series. If $K_{*}$ is the extension of an N-series $K_{+}=\left(K_{m}\right)_{m \geq 1}$, then the previous constructions specialize as follows. The target Der. $\left(\operatorname{gr}_{\bullet}\left(K_{*}\right)\right)=$ Der. $\left(\operatorname{gr}_{+}\left(K_{+}\right)\right)$of the Johnson morphism (1.5) consists of the automorphism group $\operatorname{Der}_{0}\left(\operatorname{gr}_{+}\left(K_{+}\right)\right)=\operatorname{Aut}\left(\operatorname{gr}_{+}\left(K_{+}\right)\right)$of the graded Lie algebra $\operatorname{gr}_{+}\left(K_{+}\right)$and the graded Lie algebra $\operatorname{Der}_{+}\left(\operatorname{gr}_{+}\left(K_{+}\right)\right)$of positive-degree derivations of $\mathrm{gr}_{+}\left(K_{+}\right)$.

These simplifications recover the usual Johnson homomorphisms [13, 24] and Andreadakis' constructions [1] since, if $K_{+}=\Gamma_{+} K$ is the lower central series of a free group $K$, then $\operatorname{Der}_{+}\left(\mathrm{gr}_{+}\left(K_{+}\right)\right)$is isomorphic to the Lie algebra of "truncated derivations"

$$
D_{+}\left(\operatorname{gr}_{+}\left(K_{+}\right)\right):=\bigoplus_{m \geq 1} \operatorname{Hom}\left(K_{1} / K_{2}, K_{m+1} / K_{m+2}\right) .
$$

We also consider the rational lower central series, and two mod- $p$ versions of the lower central series for a prime $p$. When $K=\pi_{1}(\Sigma)$ for a surface $\Sigma$, we recover the "mod- $p$ Johnson homomorphisms" introduced by Paris [28], Perron [32] and Cooper [6], which are suitable for the study of the mod-p Torelli group. It is the subgroup of the mapping class group consisting of elements acting trivially on $H_{1}(\Sigma ; \mathbb{Z} / p \mathbb{Z})$.

After the first version of this manuscript was released, the authors were informed that Darné, in his Ph.D. thesis in preparation [7], constructed the same generalization of the Johnson morphism for an arbitrary $N$-series acting on another $N$-series.

1.5. Extended $\mathbf{N}$-series associated to pairs of groups. We introduce two other types of extended N-series $K_{*}$, each associated with a pair $(K, N)$ of a group $K$ and a normal subgroup $N$.

First, we associate to $(K, N)$ an extended N-series $K_{*}$ defined by $K_{0}=K$ and $K_{m}=\Gamma_{m} N$ for $m \geq 1$. An important case is where $N$ is free; this happens in particular when $K$ is free. In this case, the positive part $\mathrm{gr}_{+}\left(K_{+}\right)$of the associated eg-Lie algebra gr $\bullet\left(K_{*}\right)$ is a free Lie algebra on its degree 1 part $K_{1} / K_{2}=N / \Gamma_{2} N$. Unlike the classical case where $K_{0}=K_{1}$, we have a non-trivial action of $K_{0} / K_{1}=$ $K / N$ on $\operatorname{gr}_{+}\left(K_{+}\right)$. This situation arises when we consider the action of the mapping class group of a handlebody $V_{g}$ of genus $g$ (based with a disc in the boundary) on $\pi_{1}\left(V_{g}\right)$. In fact, our study of generalized Johnson homomorphisms for extended Nseries arises from the study of this action of the handlebody mapping class group. We remark here that our generalized Johnson homomorphisms determine McNeill's "higher order Johnson homomorphisms" [20] on some subgroups of the surface mapping class group, when $N$ is any characteristic subgroup of the fundamental group $K$ of a surface.

Second, we associate to a pair $(K, N)$ with $[K, K] \leq N$ the smallest extended N-series $K_{*}$ such that $K_{0}=K_{1}=K$ and $K_{2}=N$. An example is the "weight filtration" of $K=\pi_{1}(\Sigma)$ for a punctured surface $\Sigma$; thus, we recover the generalizations of the Johnson homomorphisms on the mapping class group of $\Sigma$ studied by Asada and Nakamura [3]. In a different direction, we obtain a new notion of Johnson homomorphisms on the "Lagrangian" mapping class group of a surface studied from the point of view of finite-type invariants by Levine, who also proposed a related notion of Johnson homomorphisms [17, 18]. This will be studied in the Ph.D. thesis of Vera in connection with the "tree reduction" of the LMO functor $\widetilde{Z}$ introduced in [5]. 
1.6. Formality of extended $\mathrm{N}$-series. We show that an action of an $\mathrm{N}$-series $G_{+}$ of a group $G$ on an extended N-series $K_{*}$ of a group $K$ has an "infinitesimal" counterpart if $K_{*}$ is formal in the following sense.

The extended $\mathrm{N}$-series $K_{*}$ induces a filtration on the group algebra $\mathbb{Q}[K]$. We say that $K_{*}$ is formal if the completion of $\mathbb{Q}[K]$ with respect to this filtration is isomorphic to the degree-completion of the associated graded of $\mathbb{Q}[K]$ through an isomorphism which is the identity on the associated graded. By generalizing Quillen's result for the lower central series [35], we show that the associated graded of $\mathbb{Q}[K]$ is canonically isomorphic to the "universal enveloping algebra" of the egLie $\mathbb{Q}$-algebra $\operatorname{gr}_{\bullet}^{\mathbb{Q}}\left(K_{*}\right)$ (see Theorem 11.2). (Here $\operatorname{gr}_{\bullet}^{\mathbb{Q}}\left(K_{*}\right)$ is given by $K_{0} / K_{1}$ in degree 0 and by $\left(K_{m} / K_{m+1}\right) \otimes \mathbb{Q}$ in degree $m \geq 1$.) We can thus characterize the formality of $K_{*}$ in terms of "expansions" of $K$, generalizing the Magnus expansions for free groups. Then, we prove that such an expansion $\theta$ induces a filtrationpreserving map

$$
\varrho^{\theta}: G \longrightarrow \prod_{m \geq 1} \operatorname{Der}_{m}\left(\operatorname{gr}_{\bullet}^{\mathbb{Q}}\left(K_{*}\right)\right)
$$

which induces

$$
\bar{\tau}_{+}^{\mathbb{Q}}: \operatorname{gr}_{+}\left(G_{*}\right) \longrightarrow \operatorname{Der}_{+}\left(\operatorname{gr}_{\bullet}^{\mathbb{Q}}\left(K_{*}\right)\right),
$$

the positive part of the rational version $\bar{\tau}_{\bullet}^{\mathbb{Q}}$ of $\bar{\tau}_{\bullet}$ in (1.5) (see Theorem 12.6). Thus, we may regard the map $\varrho^{\theta}$ as an "infinitesimal version" of the action

$$
G_{+} \longrightarrow \operatorname{Aut}\left(K_{*}\right),
$$

containing all the generalized Johnson homomorphisms with coefficients in $\mathbb{Q}$.

1.7. Organization of the paper. We organize the rest of the paper as follows. In Section 2, we fix some notations about groups. Sections 3 and 4 deal with extended $\mathrm{N}$-series and extended graded Lie algebras, respectively. In Section 5, we introduce the extended graded Lie algebra consisting of the derivations of an extended graded Lie algebra. In Section 6, we construct and study the Johnson morphism induced by an extended N-series action. In Section 7, we consider truncations of the derivations of an extended graded Lie algebra. In Section 8, we specialize our constructions to N-series and, in Section 9, we illustrate these with variants of the lower central series in order to recover several versions of the Johnson homomorphisms in the literature. In Section 10, we consider two types of extended N-series defined by a pair of groups, and we announce some works in progress. Section 11 computes the associated graded of the filtration of a group algebra induced by an extended $\mathrm{N}$-series. We consider the case of formal extended N-series in Section 12.

Acknowledgments. The work of K.H. is partly supported by JSPS KAKENHI Grant Number 15K04873.

\section{Preliminaries in Group theory}

Here we recall a few facts about groups and fix some notations.

2.1. Groups. Let $G$ be a group. By $N \leq G$ we mean that $N$ is a subgroup of $G$, and by $N \triangleleft G$ that $N$ is a normal subgroup of $G$. Given a subset $S$ of $G$, let $\langle S\rangle$ denote the subgroup of $G$ generated by $S$, and $\langle\langle S\rangle\rangle=\left\langle\langle S\rangle_{G}\right.$ the normal subgroup in $G$ generated by $S$. 
For $g, h \in G$, set

$$
[g, h]=g h g^{-1} h^{-1}, \quad{ }^{g} h=g h g^{-1}, \quad h^{g}=g^{-1} h g .
$$

We will freely use the following commutator identities:

$$
\begin{gathered}
{[a, b c]=[a, b] \cdot{ }^{b}[a, c], \quad[a b, c]={ }^{a}[b, c] \cdot[a, c],} \\
{\left[a, b^{-1}\right]^{-1}=[a, b]^{b}, \quad\left[a^{-1}, b\right]^{-1}=[a, b]^{a},} \\
{\left[[a, b],{ }^{b} c\right] \cdot\left[[b, c],{ }^{c} a\right] \cdot\left[[c, a],{ }^{a} b\right]=1 .}
\end{gathered}
$$

We will need the well-known three subgroups lemma:

Lemma 2.1. If $A, B, C \leq G, N \triangleleft G$, $[A,[B, C]] \leq N$ and $[B,[C, A]] \leq N$, then we have $[C,[A, B]] \leq N$.

2.2. Group actions. Consider an action of a group $G$ on a group $K$ :

$$
G \times K \longrightarrow K, \quad(g, k) \longmapsto g(k) .
$$

Let $K \rtimes G$ denote the semidirect product of $G$ and $K$, which is the set $K \times G$ with multiplication

$$
(k, g)\left(k^{\prime}, g^{\prime}\right)=\left(k g\left(k^{\prime}\right), g g^{\prime}\right) .
$$

We naturally regard $K$ and $G$ as subgroups of $K \rtimes G$. Then, for $g \in G, k \in K$,

$$
{ }^{g} k=g k g^{-1}=g(k) \in K \leq K \rtimes G
$$

and

$$
[g, k]=g k g^{-1} k^{-1}=g(k) k^{-1} \in K \leq K \rtimes G .
$$

We will use these notations whenever a group $G$ acts on another group $K$.

For $G^{\prime} \leq G$ and $K^{\prime} \leq K$, let $\left[G^{\prime}, K^{\prime}\right]$ denote the subgroup of $K$ generated by the elements $\left[g^{\prime}, k^{\prime}\right]$ for $g^{\prime} \in G^{\prime}, k^{\prime} \in K^{\prime}$, and let ${ }^{G^{\prime}} K^{\prime}$ denote the subgroup of $K$ generated by the elements $g^{\prime} k^{\prime}$ for $g^{\prime} \in G^{\prime}, k^{\prime} \in K^{\prime}$. For $g \in G$, let $\left[g, K^{\prime}\right]$ denote the set of elements of $K$ of the form $\left[g, k^{\prime}\right]$ for all $k^{\prime} \in K^{\prime}$.

\section{Extended N-SERies AND the Johnson Filtration}

In this section, we introduce the notion of extended N-series and the Johnson filtration for an action of a group on an extended N-series.

3.1. N-series. An $N$-series [16] of a group $G$ is a descending series

$$
G=G_{1} \geq G_{2} \geq \cdots \geq G_{i} \geq \cdots
$$

such that

$$
\left[G_{m}, G_{n}\right] \leq G_{m+n} \quad \text { for } m, n \geq 1 .
$$

Note that $\left(G_{i}\right)_{i \geq 1}$ is a central series, i.e., $\left[G, G_{i}\right] \leq G_{i+1}$ for $i \geq 1$. In particular, we have $G_{i} \triangleleft G$ for $i \geq 1$.

As mentioned in the introduction, the lower central series of $G$ is the smallest N-series of $G$. 
3.2. Extended N-series. An extended $N$-series $G_{*}=\left(G_{m}\right)_{m \geq 0}$ is a descending series

$$
G_{0} \geq G_{1} \geq \cdots \geq G_{k} \geq \cdots
$$

such that

$$
\left[G_{m}, G_{n}\right] \leq G_{m+n} \quad \text { for } m, n \geq 0 .
$$

For every extended N-series $G_{*}=\left(G_{m}\right)_{m \geq 0}$, the subseries $G_{+}=\left(G_{m}\right)_{m \geq 1}$ is an $N$-series. Conversely, every N-series $\left(G_{m}\right)_{m \geq 1}$ extends to an extended $\mathrm{N}$-series by setting $G_{0}=G_{1}$.

A morphism $f: G_{*} \rightarrow K_{*}$ between extended N-series $G_{*}$ and $K_{*}$ is a homomorphism $f: G_{0} \rightarrow K_{0}$ such that $f\left(G_{m}\right) \subset K_{m}$ for all $m \geq 0$. Let eNs denote the category of extended N-series and morphisms.

In the rest of this section, we adapt several usual constructions for groups to extended $\mathrm{N}$-series.

3.3. Actions on extended $\mathbf{N}$-series. Let $K_{*}$ be an extended $\mathrm{N}$-series. By an action of an extended N-series $G_{*}$ on $K_{*}$, we mean an action of $G_{0}$ on $K_{0}$ such that

$$
\left[G_{m}, K_{n}\right] \subset K_{m+n} \quad \text { for } m, n \geq 0 .
$$

By an action of a group $G$ on $K_{*}$, we mean an action of $G$ on $K_{0}$ such that

$$
g\left(K_{n}\right)=K_{n} \quad \text { for } g \in G, n \geq 0 .
$$

Note that if $G_{*}$ acts on $K_{*}$, then $G_{0}$ acts on $K_{*}$.

3.4. Johnson filtrations. If a group $G$ acts on an extended N-series $K_{*}$, then we have an extended N-series $\mathcal{F}_{*}^{K_{*}}(G)$ of $G$ defined by

$$
\mathcal{F}_{m}^{K_{*}}(G)=\left\{g \in G \mid\left[g, K_{n}\right] \subset K_{m+n} \text { for } n \geq 0\right\}
$$

for every $m \geq 0$, which we call the Johnson filtration of $G$ induced by $K_{*}$.

Proposition 3.1. If a group $G$ acts on an extended $N$-series $K_{*}$, then the Johnson filtration $\mathcal{F}_{*}^{K_{*}}(G)$ is the largest extended $N$-series of $G$ acting on $K_{*}$.

Proof. Set $G_{*}=\mathcal{F}_{*}^{K_{*}}(G)$. One easily checks that $G_{*}$ is a descending series of $G$, and that $\left[G_{m}, K_{n}\right] \subset K_{m+n}$ for $m, n \geq 0$. We have $\left[G_{m}, G_{n}\right] \subset G_{m+n}$ for $m, n \geq 0$, since for $i \geq 0$

$$
\begin{aligned}
{\left[\left[G_{m}, G_{n}\right], K_{i}\right] } & \subset\left\langle\left\langle\left[G_{m},\left[G_{n}, K_{i}\right]\right] \cdot\left[G_{n},\left[G_{m}, K_{i}\right]\right]\right\rangle\right\rangle_{K_{0} \rtimes G} \quad \text { (by Lemma 2.1) } \\
& \subset\left\langle\left\langle\left[G_{m}, K_{n+i}\right] \cdot\left[G_{n}, K_{m+i}\right]\right\rangle\right\rangle_{K_{0} \rtimes G} \\
& \subset\left\langle\left\langle K_{m+n+i}\right\rangle_{K_{0} \rtimes G}=K_{m+n+i} .\right.
\end{aligned}
$$

Hence $G_{*}$ is an extended N-series acting on $K_{*}$. It is clear from the definition of $G_{*}$ that, if $G_{*}^{\prime}$ is another extended N-series of $G$ acting on $K_{*}$, then $G_{m}^{\prime} \leq G_{m}$.

Remark 3.2. In the proof of Proposition 3.1, we did not use the condition $\left[K_{m}, K_{n}\right] \leq$ $K_{m+n}, m, n \geq 0$. Therefore, we can generalize Proposition 3.1 to any normal series $K_{*}=\left(K_{m}\right)_{m \geq 0}$ of a group $K$. 
3.5. Automorphism group of an extended $\mathbf{N}$-series. Let $K_{*}$ be an extended $\mathrm{N}$-series. Define the automorphism group of $K_{*}$ by

$$
\operatorname{Aut}\left(K_{*}\right)=\left\{g \in \operatorname{Aut}\left(K_{0}\right) \mid g\left(K_{i}\right)=K_{i} \text { for } i \geq 0\right\},
$$

which is the largest subgroup of $\operatorname{Aut}\left(K_{0}\right)$ acting on $K_{*}$. Note that a homomorphism $G \rightarrow \operatorname{Aut}\left(K_{*}\right)$ is equivalent to an action of $G$ on $K_{*}$.

Let $\operatorname{Aut}_{*}\left(K_{*}\right)$ denote the Johnson filtration $\mathcal{F}_{*}^{K_{*}}\left(\operatorname{Aut}\left(K_{*}\right)\right)$ of $\operatorname{Aut}\left(K_{*}\right)$ induced by $K_{*}$; thus,

$$
\operatorname{Aut}_{m}\left(K_{*}\right)=\left\{g \in \operatorname{Aut}\left(K_{*}\right) \mid\left[g, K_{n}\right] \subset K_{m+n} \text { for } n \geq 0\right\}
$$

for $m \geq 0$. Note that a morphism $G_{*} \rightarrow \operatorname{Aut}_{*}\left(K_{*}\right)$ of extended N-series is equivalent to an action of $G_{*}$ on $K_{*}$. The following lemma is easily verified.

Lemma 3.3. Let $K_{*}$ be an extended $N$-series.

(1) If $K_{m}$ is characteristic in $K_{0}$ for all $m \geq 1$, then $\operatorname{Aut}\left(K_{*}\right)=\operatorname{Aut}\left(K_{0}\right)$.

(2) If $K_{m}$ is characteristic in $K_{1}$ for all $m \geq 2$, then $\operatorname{Aut}\left(K_{*}\right)=\operatorname{Aut}\left(K_{0}, K_{1}\right)$, where $\operatorname{Aut}\left(K_{0}, K_{1}\right)=\left\{g \in \operatorname{Aut}\left(K_{0}\right) \mid g\left(K_{1}\right)=K_{1}\right\}$.

Example 3.4. Let $K_{*}$ be an extended N-series. Then $K_{*}$ acts on itself via the conjugation $K \times K \rightarrow K,\left(k, k^{\prime}\right) \mapsto{ }^{k} k^{\prime}$. Thus, we have a morphism of extended $\mathrm{N}$-series

$$
\operatorname{Ad}^{K_{*}}: K_{*} \longrightarrow \operatorname{Aut}_{*}\left(K_{*}\right)
$$

called the adjoint action of $K_{*}$. In general, $K_{*}$ does not coincide with the Johnson filtration $\mathcal{F}_{*}^{K_{*}}\left(K_{0}\right)$ of $K_{0}$ induced by its action on $K_{*}$. For example, if $K_{0}$ is abelian, then $\mathcal{F}_{*}^{K_{*}}\left(K_{0}\right)=\left(K_{0}\right)_{n \geq 0}$, which is different from $K_{*}$ in general. See Remark 10.4 for an example where we have $K_{*}=\mathcal{F}_{*}^{K_{*}}\left(K_{0}\right)$.

\section{Extended GRaded Lie algebras}

It is well known [16] that to each $\mathrm{N}$-series is associated a graded Lie algebra over $\mathbb{Z}$. Here we associate to each extended $\mathrm{N}$-series an eg-Lie algebra.

4.1. Graded Lie algebras. Recall that a graded Lie algebra $L_{+}=\left(L_{m}\right)_{m \geq 1}$ consists of abelian groups $L_{m}, m \geq 1$, and bilinear maps

$$
[\cdot, \cdot]: L_{m} \times L_{n} \rightarrow L_{m+n}
$$

for $m, n \geq 1$ such that

- $[x, x]=0$ for $x \in L_{m}, m \geq 1$,

- $[x, y]+[y, x]=0$ for $x \in L_{m}, y \in L_{n}, m, n \geq 1$,

- $[x,[y, z]]+[y,[z, x]]+[z,[x, y]]=0$ for $x \in L_{m}, y \in L_{n}, z \in L_{p}, m, n, p \geq 1$.

Also, let $L_{+}$denote the direct sum $\bigoplus_{m \geq 1} L_{m}$ by abuse of notation.

A morphism $f_{+}: L_{+} \rightarrow L_{+}^{\prime}$ of graded Lie algebras is a family $f_{+}=\left(f_{i}\right)_{i \geq 1}$ of homomorphisms $f_{i}: L_{i} \rightarrow L_{i}^{\prime}$ such that $f_{i+j}([x, y])=\left[f_{i}(x), f_{j}(y)\right]$ for all $x \in L_{i}$, $y \in L_{j}, i, j \geq 1$. An automorphism of $L_{+}$is an invertible morphism from $L_{+}$to itself. Let $\operatorname{Aut}\left(L_{+}\right)$denote the group of automorphisms of $L_{+}$.

An action of a group $G$ on $L_{+}$is a homomorphism from $G$ to $\operatorname{Aut}\left(L_{+}\right)$. In other words, it is a degree-preserving action $(g, x) \mapsto{ }^{g} x$ of $G$ on $L_{+}$such that

$$
{ }^{g}[x, y]=\left[{ }^{g} x,{ }^{g} y\right] \quad \text { for } g \in G \text { and } x, y \in L_{+} .
$$


4.2. Extended graded Lie algebras. An extended graded Lie algebra (abbreviated as eg-Lie algebra) $L_{\bullet}=\left(L_{m}\right)_{m \geq 0}$ consists of

- a group $L_{0}$,

- a graded Lie algebra $L_{+}=\left(L_{m}\right)_{m \geq 1}$,

- an action $(g, x) \mapsto{ }^{g} x$ of $L_{0}$ on $L_{+}$.

A morphism $f_{\bullet}=\left(f_{m}: L_{m} \rightarrow L_{m}^{\prime}\right)_{m \geq 0}: L_{\bullet} \rightarrow L_{\bullet}^{\prime}$ between eg-Lie algebras $L \bullet$ and $L_{\bullet}^{\prime}$ consists of

- a homomorphism $f_{0}: L_{0} \rightarrow L_{0}^{\prime}$,

- a graded Lie algebra morphism $f_{+}=\left(f_{m}\right)_{m \geq 1}: L_{+} \rightarrow L_{+}^{\prime}$,

such that

$$
f_{m}\left({ }^{x} y\right)={ }^{f_{0}}(x)\left(f_{m}(y)\right)
$$

for all $x \in L_{0}, y \in L_{m}, m \geq 1$. Let egL denote the category of eg-Lie algebras and morphisms.

4.3. From extended $\mathrm{N}$-series to eg-Lie algebras. For each extended $\mathrm{N}$-series $K_{*}$, we define the associated eg-Lie algebra $\bar{K}_{\bullet}=\operatorname{gr}_{\bullet}\left(K_{*}\right)$ as follows. Set

$$
\bar{K}_{m}=\operatorname{gr}_{m}\left(K_{*}\right)=K_{m} / K_{m+1}
$$

for all $m \geq 0$. The group $\bar{K}_{0}$ is not abelian in general, whereas $\bar{K}_{m}$ is abelian for $m \geq 1$. Thus we will use multiplicative notation for the former, and the additive notation for the latter. The Lie bracket $[\cdot, \cdot]: \bar{K}_{m} \times \bar{K}_{n} \rightarrow \bar{K}_{m+n}$ in $\bar{K} \bullet$ is given by

$$
\left[a K_{m+1}, b K_{n+1}\right]=[a, b] K_{m+n+1}
$$

for $m, n \geq 1$, and the action of $\bar{K}_{0}$ on $\bar{K}_{m}$ is given by

$$
{ }^{\left(a K_{1}\right)}\left(b K_{m+1}\right)=\left({ }^{a} b\right) K_{m+1} .
$$

Observe that $\bar{K}_{+}$is the usual graded Lie algebra associated to the N-series $K_{+}$(see [16, Theorem 2.1]).

There is a functor gr $:$ eNs $\rightarrow$ egL. Indeed, every morphism $f: G_{*} \rightarrow K_{*}$ in eNs induces a morphism gr $\bullet(f): \operatorname{gr}_{\bullet}\left(G_{*}\right) \rightarrow \operatorname{gr}_{\bullet}\left(K_{*}\right)$ in egL defined by

$$
\operatorname{gr} \bullet(f)\left(g G_{m+1}\right)=f(g) K_{m+1}, \quad\left(g \in G_{m}, m \geq 0\right) .
$$

\section{Derivation eg-Lie algebras of Eg-Lie algebras}

In this section, we introduce the derivation eg-Lie algebra of an eg-Lie algebra, which generalizes the derivation Lie algebra of a graded Lie algebra.

5.1. Derivations of an eg-Lie algebra. Let $L$ • be an eg-Lie algebra.

Definition 5.1. Let $m \geq 1$. A derivation $d=\left(d_{i}\right)_{i \geq 0}$ of $L$ • of degree $m$ is a family of maps $d_{i}: L_{i} \rightarrow L_{m+i}$ satisfying the following conditions.

(1) $d_{+}=\left(d_{i}\right)_{i \geq 1}$ is a derivation of the graded Lie algebra $L_{+}$, i.e., the $d_{i}$ for $i \geq 1$ are homomorphisms such that

$$
d_{i+j}([a, b])=\left[d_{i}(a), b\right]+\left[a, d_{j}(b)\right]
$$

for $a \in L_{i}, b \in L_{j}, i, j \geq 1$.

(2) The map $d_{0}: L_{0} \rightarrow L_{m}$ is a 1-cocycle. In other words, we have

$$
d_{0}(a b)=d_{0}(a)+{ }^{a}\left(d_{0}(b)\right)
$$

for $a, b \in L_{0}$. 
(3) We have

$$
d_{i}\left({ }^{a} b\right)=\left[d_{0}(a),{ }^{a} b\right]+{ }^{a}\left(d_{i}(b)\right)
$$

for $a \in L_{0}, b \in L_{i}, i \geq 1$.

For $m \geq 1$, let $\operatorname{Der}_{m}\left(L_{\bullet}\right)$ be the group of derivations of $L_{\bullet}$ of degree $m$. Set $\operatorname{Der}_{+}\left(L_{\bullet}\right)=\left(\operatorname{Der}_{m}\left(L_{\bullet}\right)\right)_{m \geq 1}$.

Theorem 5.2. We have a graded Lie algebra structure on $\operatorname{Der}_{+}\left(L_{\bullet}\right)$ such that, for $m, n \geq 1$, the Lie bracket

$$
[\cdot, \cdot]: \operatorname{Der}_{m}\left(L_{\bullet}\right) \times \operatorname{Der}_{n}\left(L_{\bullet}\right) \longrightarrow \operatorname{Der}_{m+n}\left(L_{\bullet}\right)
$$

is given by

$$
\left[d, d^{\prime}\right]_{i}(a)= \begin{cases}d_{n}\left(d_{0}^{\prime}(a)\right)-d_{m}^{\prime}\left(d_{0}(a)\right)-\left[d_{0}(a), d_{0}^{\prime}(a)\right] & \left(i=0, a \in L_{0}\right), \\ d_{n+i}\left(d_{i}^{\prime}(a)\right)-d_{m+i}^{\prime}\left(d_{i}(a)\right) & \left(i \geq 1, a \in L_{i}\right) .\end{cases}
$$

We call $\operatorname{Der}_{+}\left(L_{\bullet}\right)$ the derivation graded Lie algebra of $L_{\bullet}$.

Proof of Theorem 5.2. For simplicity of notation, set $D_{+}=\operatorname{Der}_{+}\left(L_{\bullet}\right)$.

For $d \in D_{m}, d^{\prime} \in D_{n}, m, n \geq 1$, define $\left[d, d^{\prime}\right]=\left(\left[d, d^{\prime}\right]_{i}: L_{i} \rightarrow L_{i+m+n}\right)_{i \geq 0}$ by (5.1). We prove $\left[d, d^{\prime}\right] \in D_{m+n}$ as follows.

First, $\left[d, d^{\prime}\right]_{+}=\left(\left[d, d^{\prime}\right]_{i}\right)_{i \geq 1}$ is a derivation of $L_{+}$since the commutator of two derivations of a Lie algebra is a derivation.

Second, we verify that $\left[d, d^{\prime}\right]_{0}: L_{0} \rightarrow L_{m+n}$ is a 1 -cocycle. For $a, b \in L_{0}$,

$$
\begin{aligned}
& {\left[d, d^{\prime}\right](a b) } \\
= & d d^{\prime}(a b)-d^{\prime} d(a b)-\left[d(a b), d^{\prime}(a b)\right] \\
= & d\left(d^{\prime}(a)+{ }^{a}\left(d^{\prime}(b)\right)\right)-d d^{\prime}\left(d(a)+{ }^{a}(d(b))\right)-\left[d(a)+{ }^{a}(d(b)), d^{\prime}(a)+{ }^{a}\left(d^{\prime}(b)\right)\right] \\
= & d d^{\prime}(a)+\left[d(a),{ }^{a}\left(d^{\prime}(b)\right)\right]+{ }^{a}\left(d d^{\prime}(b)\right)-d^{\prime} d(a)-\left[d^{\prime}(a),{ }^{a}(d(b))\right]-{ }^{a}\left(d^{\prime} d(b)\right) \\
& -\left[d(a), d^{\prime}(a)\right]-\left[d(a),{ }^{a}\left(d^{\prime}(b)\right)\right]-\left[{ }^{a}(d(b)), d^{\prime}(a)\right]-\left[{ }^{a}(d(b)),{ }^{a}\left(d^{\prime}(b)\right)\right] \\
= & d d^{\prime}(a)+{ }^{a}\left(d d^{\prime}(b)\right)-d^{\prime} d(a)-{ }^{a}\left(d^{\prime} d(b)\right)-\left[d(a), d^{\prime}(a)\right]-{ }^{a}\left[d(b), d^{\prime}(b)\right] \\
= & {\left[d, d^{\prime}\right](a)+{ }^{a}\left(\left[d, d^{\prime}\right](b)\right) . }
\end{aligned}
$$

Third, for $a \in L_{0}, b \in L_{i}, i \geq 1$, we have

$$
\begin{aligned}
{\left[d, d^{\prime}\right]\left({ }^{a} b\right)=} & d d^{\prime}\left({ }^{a} b\right)-d^{\prime} d\left({ }^{a} b\right) \\
= & d\left(\left[d^{\prime}(a),{ }^{a} b\right]+{ }^{a}\left(d^{\prime}(b)\right)\right)-d^{\prime}\left(\left[d(a),{ }^{a} b\right]+{ }^{a}(d(b))\right) \\
= & {\left[d d^{\prime}(a),{ }^{a} b\right]+\left[d^{\prime}(a), d\left({ }^{a} b\right)\right]+\left[d(a),{ }^{a}\left(d^{\prime}(b)\right)\right]+{ }^{a}\left(d d^{\prime}(b)\right) } \\
& -\left[d^{\prime} d(a),{ }^{a} b\right]-\left[d(a), d^{\prime}\left({ }^{a} b\right)\right]-\left[d^{\prime}(a),{ }^{a}(d(b))\right]-{ }^{a}\left(d^{\prime} d(b)\right) \\
= & {\left[d d^{\prime}(a),{ }^{a} b\right]+\left[d^{\prime}(a),\left[d(a),{ }^{a} b\right]+{ }^{a}(d(b))\right]+\left[d(a),{ }^{a}\left(d^{\prime}(b)\right)\right]+{ }^{a}\left(d d^{\prime}(b)\right) } \\
& -\left[d^{\prime} d(a),{ }^{a} b\right]-\left[d(a),\left[d^{\prime}(a),{ }^{a} b\right]+{ }^{a}\left(d^{\prime}(b)\right)\right]-\left[d^{\prime}(a),{ }^{a}(d(b))\right]-{ }^{a}\left(d^{\prime} d(b)\right) \\
= & {\left[d d^{\prime}(a),{ }^{a} b\right]+\left[d^{\prime}(a),\left[d(a),{ }^{a} b\right]\right]+{ }^{a}\left(d d^{\prime}(b)\right) } \\
& -\left[d^{\prime} d(a),{ }^{a} b\right]-\left[d(a),\left[d^{\prime}(a),{ }^{a} b\right]\right]-{ }^{a}\left(d^{\prime} d(b)\right) \\
= & {\left[d d^{\prime}(a)-d^{\prime} d(a)-\left[d(a), d^{\prime}(a)\right],,^{a} b\right]+{ }^{a}\left(d d^{\prime}(b)-d^{\prime} d(b)\right) } \\
= & {\left[\left[d, d^{\prime}\right](a),{ }^{a} b\right]+{ }^{a}\left(\left[d, d^{\prime}\right](b)\right) . }
\end{aligned}
$$

Therefore, $\left[d, d^{\prime}\right]$ is a derivation of $L_{\bullet}$ of degree $m+n$. 
Now we show that the maps $[\cdot, \cdot]: D_{m} \times D_{n} \rightarrow D_{m+n}$ for $m, n \geq 1$ define a graded Lie algebra structure on $D_{+}$. Clearly, we have $[d, d]=0$ and $\left[d, d^{\prime}\right]+\left[d^{\prime}, d\right]=0$ for $d, d^{\prime} \in D_{+}$. Thus it remains to check the Jacobi identity

$$
\left[d,\left[d^{\prime}, d^{\prime \prime}\right]\right](a)+\left[d^{\prime \prime},\left[d, d^{\prime}\right]\right](a)+\left[d^{\prime},\left[d^{\prime \prime}, d\right]\right](a)=0
$$

for $d, d^{\prime}, d^{\prime \prime} \in D_{+}$and $a \in L_{i}$ with $i \geq 0$. For $i \geq 1$, this is the standard fact that derivations of a Lie algebra form a Lie algebra. For $i=0$, we have

$$
\begin{aligned}
{\left[d,\left[d^{\prime}, d^{\prime \prime}\right]\right](a)=} & d\left[d^{\prime}, d^{\prime \prime}\right](a)-\left[d^{\prime}, d^{\prime \prime}\right] d(a)-\left[d(a),\left[d^{\prime}, d^{\prime \prime}\right](a)\right] \\
= & d\left(d^{\prime} d^{\prime \prime}(a)-d^{\prime \prime} d^{\prime}(a)-\left[d^{\prime}(a), d^{\prime \prime}(a)\right]\right)-\left(d^{\prime} d^{\prime \prime} d(a)-d^{\prime \prime} d^{\prime} d(a)\right) \\
& -\left[d(a), d^{\prime} d^{\prime \prime}(a)-d^{\prime \prime} d^{\prime}(a)-\left[d^{\prime}(a), d^{\prime \prime}(a)\right]\right] \\
= & d d^{\prime} d^{\prime \prime}(a)-d d^{\prime \prime} d^{\prime}(a)-\left[d d^{\prime}(a), d^{\prime \prime}(a)\right]-\left[d^{\prime}(a), d d^{\prime \prime}(a)\right] \\
& -d^{\prime} d^{\prime \prime} d(a)+d^{\prime \prime} d^{\prime} d(a) \\
& -\left[d(a), d^{\prime} d^{\prime \prime}(a)\right]+\left[d(a), d^{\prime \prime} d^{\prime}(a)\right]+\left[d(a),\left[d^{\prime}(a), d^{\prime \prime}(a)\right]\right]
\end{aligned}
$$

from which (5.2) follows.

\subsection{Derivation eg-Lie algebras. Let $L$ • be an eg-Lie algebra.}

Theorem 5.3. The derivation graded Lie algebra $\operatorname{Der}_{+}\left(L_{\bullet}\right)$ extends to an eg-Lie algebra $\operatorname{Der}\left(L_{\bullet}\right)$ by setting $\operatorname{Der}_{0}\left(L_{\bullet}\right)=\operatorname{Aut}\left(L_{\bullet}\right)$ and by defining an action

$$
\operatorname{Der}_{0}\left(L_{\bullet}\right) \times \operatorname{Der}_{m}\left(L_{\bullet}\right) \longrightarrow \operatorname{Der}_{m}\left(L_{\bullet}\right), \quad(f, d) \longmapsto{ }^{f} d,
$$

for $m \geq 1$ by

$$
\left({ }^{f} d\right)_{i}(a)=f_{m+i} d_{i} f_{i}^{-1}(a) \quad\left(i \geq 0, a \in L_{i}\right) .
$$

We call Der $\left(L_{\bullet}\right)=\left(\operatorname{Der}_{m}\left(L_{\bullet}\right)\right)_{m \geq 0}$ the derivation eg-Lie algebra of $L_{\bullet}$.

Proof. For simplicity of notation, set $D_{\bullet}=\operatorname{Der}_{\bullet}\left(L_{\bullet}\right)$. For $f \in D_{0}, d \in D_{m}, m \geq 1$, define ${ }^{f} d=\left(\left({ }^{f} d\right)_{i}: L_{i} \rightarrow L_{i+m}\right)_{i \geq 0}$ by (5.4). We prove ${ }^{f} d \in D_{m}$ as follows.

First, we check that $\left({ }^{f} d\right)_{+}$is a derivation of $L_{+}$. For $a \in L_{i}, b \in L_{j}, i, j \geq 1$,

$$
\begin{aligned}
\left({ }^{f} d\right)([a, b]) & =f d f^{-1}([a, b]) \\
& =f d\left(\left[f^{-1}(a), f^{-1}(b)\right]\right) \\
& =f\left(\left[d f^{-1}(a), f^{-1}(b)\right]+\left[f^{-1}(a), d f^{-1}(b)\right]\right) \\
& =\left[f d f^{-1}(a), b\right]+\left[a, f d f^{-1}(b)\right] \\
& =\left[\left({ }^{f} d\right)(a), b\right]+\left[a,\left({ }^{f} d\right)(b)\right] .
\end{aligned}
$$

Second, we check that $\left({ }^{f} d\right)_{0}: L_{0} \rightarrow L_{m}$ is a 1-cocycle. For $a, b \in L_{0}$,

$$
\begin{aligned}
\left({ }^{f} d\right)(a b) & =f d f^{-1}(a b) \\
& =f d\left(f^{-1}(a) f^{-1}(b)\right) \\
& =f\left(d f^{-1}(a)+{ }^{-1}(a)\left(d f^{-1}(b)\right)\right) \\
& =f d f^{-1}(a)+{ }^{a}\left(f d f^{-1}(b)\right)=\left({ }^{f} d\right)(a)+{ }^{a}\left(\left({ }^{f} d\right)(b)\right) .
\end{aligned}
$$


Third, we have for $a \in L_{0}, b \in L_{i}, i \geq 1$,

$$
\begin{aligned}
\left({ }^{f} d\right)\left({ }^{a} b\right) & =f d f^{-1}\left({ }^{a} b\right) \\
& =f d\left(f^{-1}(a)\left(f^{-1}(b)\right)\right) \\
& =f\left(\left[d f^{-1}(a),{ }^{-1}(a)\left(f^{-1}(b)\right)\right]+f^{-1}(a)\left(d f^{-1}(b)\right)\right) \\
& =\left[f d f^{-1}(a),{ }^{a} b\right]+{ }^{a}\left(f d f^{-1}(b)\right) \\
& =\left[\left({ }^{f} d\right)(a),{ }^{a} b\right]+{ }^{a}\left(\left({ }^{f} d\right)(b)\right) .
\end{aligned}
$$

Therefore, ${ }^{f} d$ is a derivation of the eg-Lie algebra $L_{\bullet}$ of degree $m$.

It is easy to check that the maps $D_{0} \times D_{m} \rightarrow D_{m},(f, d) \mapsto{ }^{f} d$ for $m \geq 1$ form an action of $D_{0}$ on the graded abelian group $D_{+}$. Let us verify that this action preserves the Lie bracket of $D_{+}$. Let $g \in D_{0}, d \in D_{m}, d^{\prime} \in D_{n}$ with $m, n \geq 1$, and let $a \in L_{i}$ with $i \geq 0$. For $i \geq 1$, we have

$$
\begin{aligned}
\left.{ }^{g}\left[d, d^{\prime}\right]\right)(a) & =g\left[d, d^{\prime}\right] g^{-1}(a) \\
& =g\left(d d^{\prime}-d^{\prime} d\right) g^{-1}(a)=\left(g d g^{-1} g d^{\prime} g^{-1}-g d^{\prime} g^{-1} g d g^{-1}\right)(a)=\left[{ }^{g} d,{ }^{g} d^{\prime}\right](a)
\end{aligned}
$$

and, for $i=0$, we have

$$
\begin{aligned}
\left({ }^{g}\left[d, d^{\prime}\right]\right)(a) & =g\left[d, d^{\prime}\right] g^{-1}(a) \\
& =g d d^{\prime} g^{-1}(a)-g d^{\prime} d g^{-1}(a)-g\left[d g^{-1}(a), d^{\prime} g^{-1}(a)\right] \\
& =g d g^{-1} g d^{\prime} g^{-1}(a)-g d^{\prime} g^{-1} g d g^{-1}(a)-\left[g d g^{-1}(a), g d^{\prime} g^{-1}(a)\right] \\
& \left.={ }^{g} d{ }^{g} d^{\prime}\right](a) .
\end{aligned}
$$

Hence $D_{\bullet}$ is an eg-Lie algebra.

Example 5.4. Let $L$ • be an eg-Lie algebra. There is a morphism of eg-Lie algebras

$$
\operatorname{ad}=\operatorname{ad}^{L} \bullet: L_{\bullet} \longrightarrow \operatorname{Der} \bullet\left(L_{\bullet}\right),
$$

called the adjoint action of $L_{\bullet}$. It is defined by

$$
\operatorname{ad}(a)(b)= \begin{cases}{ }^{a} b & \text { for } a \in L_{0}, b \in L_{n}, n \geq 0 \\ {[a, b]} & \text { for } a \in L_{m}, m \geq 1, b \in L_{n}, n \geq 0\end{cases}
$$

where we set $[a, b]=a-{ }^{b} a$ for $a \in L_{m}, m \geq 1$ and $b \in L_{0}$. The proof is straightforward and left to the reader.

\section{The Johnson homomorphisms of An EXTENDED N-SERIES ACtion}

In this section, we generalize Johnson homomorphisms for an arbitrary action of extended N-series $G_{*}$ on $K_{*}$. These "Johnson homomorphisms" form a "Johnson morphism"

$$
\bar{\tau}_{\bullet}: \operatorname{gr}_{\bullet}\left(G_{*}\right) \longrightarrow \operatorname{Der} \bullet\left(\operatorname{gr}_{\bullet}\left(K_{*}\right)\right)
$$

with values in the derivation eg-Lie algebra of $\operatorname{gr}_{\bullet}\left(K_{*}\right)$. 
6.1. Generalized Johnson homomorphisms. In this subsection, we consider an extended N-series $G_{*}$ acting on an extended $\mathrm{N}$-series $K_{*}$, and we set $\bar{K}_{\bullet}=\operatorname{gr}_{\bullet}\left(K_{*}\right)$. For every $m \geq 0$, we will define a homomorphism

$$
\tau_{m}=\tau_{m}^{G_{*}, K_{*}}: G_{m} \longrightarrow \operatorname{Der}_{m}\left(\bar{K}_{\bullet}\right),
$$

which we call the mth (generalized) Johnson homomorphism. We treat the cases $m=0$ and $m>0$ separately.

Proposition 6.1. There is a homomorphism

$$
\begin{gathered}
\tau_{0}: G_{0} \longrightarrow \operatorname{Aut}\left(\bar{K}_{\bullet}\right) \\
\text { which maps each } g \in G_{0} \text { to } \tau_{0}(g)=\left(\tau_{0}(g)_{i}: \bar{K}_{i} \rightarrow \bar{K}_{i}\right)_{i \geq 0} \text { defined by } \\
\tau_{0}(g)_{i}\left(a K_{i+1}\right)=\left({ }^{g} a\right) K_{i+1} .
\end{gathered}
$$

Proof. Let $\operatorname{End}\left(\bar{K}_{\bullet}\right)$ denote the monoid of endomorphisms of the eg-Lie algebra $\bar{K}_{\bullet}$. Let $g \in G_{0}$. We prove that $\tau_{0}(g) \in \operatorname{End}\left(\bar{K}_{\bullet}\right)$ is well defined as follows. It is easy to see that $\tau_{0}(g)_{i}: \bar{K}_{i} \rightarrow \bar{K}_{i}$ is a well-defined homomorphism for $i \geq 0$.

Next, $\left(\tau_{0}(g)_{i}\right)_{i \geq 1}: \bar{K}_{+} \rightarrow \bar{K}_{+}$is a graded Lie algebra automorphism since, for $a \in K_{i}, b \in K_{j}, i, j \geq 1$, we have

$$
\begin{aligned}
\tau_{0}(g)\left(\left[a K_{i+1}, b K_{j+1}\right]\right) & =\tau_{0}(g)\left([a, b] K_{i+j+1}\right)=\left({ }^{g}[a, b]\right) K_{i+j+1}=\left[{ }^{g} a,{ }^{g} b\right] K_{i+j+1} \\
& =\left[\left({ }^{g} a\right) K_{i+1},\left({ }^{g} b\right) K_{j+1}\right]=\left[\tau_{0}(g)\left(a K_{i+1}\right), \tau_{0}(g)\left(b K_{j+1}\right)\right] .
\end{aligned}
$$

We now check the equivariance property:

$$
\begin{aligned}
\tau_{0}(g)\left({ }^{\left(a K_{1}\right)}\left(b K_{i+1}\right)\right) & \left.=\tau_{0}(g)\left(\left({ }^{a} b\right) K_{i+1}\right)=\left({ }^{g}\left({ }^{a} b\right)\right) K_{i+1}=\left({ }^{g} a\right)\left({ }^{g} b\right)\right) K_{i+1} \\
& ={ }^{\left({ }^{g} a\right) K_{1}}\left(\left({ }^{g} b\right) K_{i+1}\right)={ }^{\tau_{0}(g)\left(a K_{1}\right)}\left(\tau_{0}(g)\left(b K_{i+1}\right)\right)
\end{aligned}
$$

for $a \in K_{0}, b \in K_{i}, i \geq 1$. Thus, we have $\tau_{0}(g) \in \operatorname{End}\left(\bar{K}_{\bullet}\right)$.

The map $\tau_{0}: G_{0} \rightarrow \operatorname{End}\left(\bar{K}_{\bullet}\right)$ is a monoid homomorphism, i.e., we have $\tau_{0}\left(g g^{\prime}\right)=$ $\tau_{0}(g) \tau_{0}\left(g^{\prime}\right)$ for $g, g^{\prime} \in G_{0}$. Indeed, for $a \in K_{i}, i \geq 0$, we have

$$
\begin{aligned}
\tau_{0}\left(g g^{\prime}\right)\left(a K_{i+1}\right) & =\left({ }^{\left(g g^{\prime}\right)} a\right) K_{i+1}=\left({ }^{g}\left({ }^{g^{\prime}} a\right)\right) K_{i+1}=\tau_{0}(g)\left(\left({ }^{g^{\prime}} a\right) K_{i+1}\right) \\
& =\tau_{0}(g)\left(\tau_{0}\left(g^{\prime}\right)\left(a K_{i+1}\right)\right)=\left(\tau_{0}(g) \tau_{0}\left(g^{\prime}\right)\right)\left(a K_{i+1}\right) .
\end{aligned}
$$

Hence $\tau_{0}$ takes values in $\operatorname{Aut}\left(\bar{K}_{\bullet}\right)$.

Proposition 6.2. For $m \geq 1$, there is a homomorphism

$$
\tau_{m}: G_{m} \longrightarrow \operatorname{Der}_{m}\left(\bar{K}_{\bullet}\right)
$$

which maps each $g \in G_{m}$ to $\tau_{m}(g)=\left(\tau_{m}(g)_{i}: \bar{K}_{i} \rightarrow \bar{K}_{m+i}\right)_{i \geq 0}$ defined by

$$
\tau_{m}(g)_{i}\left(a K_{i+1}\right)=[g, a] K_{m+i+1} .
$$

Proof. Let $g \in G_{m}$. We show that $\tau_{m}(g) \in \operatorname{Der}_{m}\left(\bar{K}_{\bullet}\right)$ is well defined as follows.

Since $G_{*}$ acts on $K_{*}$, we easily see that the map $\tau_{m}(g)_{i}: \bar{K}_{i} \rightarrow \bar{K}_{m+i}$ is well defined by (6.2) for all $i \geq 0$. The map $\tau_{m}(g)_{i}: \bar{K}_{i} \rightarrow \bar{K}_{m+i}$ is a 1-cocycle if $i=0$ and a homomorphism if $i \geq 1$ : indeed, for all $a, b \in K_{i}$, we have

$$
\begin{aligned}
& \tau_{m}(g)\left(\left(a K_{i+1}\right)\left(b K_{i+1}\right)\right)=\tau_{m}(g)\left(a b K_{i+1}\right) \\
& =[g, a b] K_{m+i+1} \\
& =\left([g, a] \cdot{ }^{a}[g, b]\right) K_{m+i+1} \\
& = \begin{cases}\tau_{m}(g)(a)+{ }^{\left(a K_{1}\right)}\left(\tau_{m}(g)(b)\right) & \text { if } i=0, \\
\tau_{m}(g)(a)+\tau_{m}(g)(b) & \text { if } i \geq 1 .\end{cases}
\end{aligned}
$$


Next, we verify that $\left(\tau_{m}(g)_{i}\right)_{i \geq 1}$ is a derivation of $\bar{K}_{+}$. For $a \in K_{i}, b \in K_{j}$, $i, j \geq 1$, we have

$$
\begin{aligned}
\tau_{m}(g)\left(\left[a K_{i+1}, b K_{j+1}\right]\right) & =\tau_{m}(g)\left([a, b] K_{i+j+1}\right) \\
& =[g,[a, b]] K_{m+i+j+1} \\
& =\left([[g, a], b] K_{m+i+j+1}\right)+\left([a,[g, b]] K_{m+i+j+1}\right) \\
& =\left[[g, a] K_{m+i+1}, b K_{j+1}\right]+\left[a K_{i+1},[g, b] K_{m+j+1}\right] \\
& =\left[\tau_{m}(g)\left(a K_{i+1}\right), b K_{j+1}\right]+\left[a K_{i+1}, \tau_{m}(g)\left(b K_{j+1}\right)\right] .
\end{aligned}
$$

It remains to check that

(6.3) $\tau_{m}(g)\left({ }^{\left(a K_{1}\right)}\left(b K_{i+1}\right)\right)=\left[\tau_{m}(g)\left(a K_{1}\right),{ }^{\left(a K_{1}\right)}\left(b K_{i+1}\right)\right]+{ }^{\left(a K_{1}\right)}\left(\tau_{m}(g)\left(b K_{i+1}\right)\right)$

for $a \in K_{0}$ and $b \in K_{i}, i \geq 1$. Indeed, since

$$
\begin{aligned}
& { }^{a}[g, b]=\left[{ }^{a} g,{ }^{a} b\right]=\left[[a, g] g,{ }^{a} b\right]={ }^{a a, g]}\left[g,{ }^{a} b\right] \cdot\left[[a, g],{ }^{a} b\right] \\
& \equiv\left[g,{ }^{a} b\right] \cdot\left[[g, a]^{-1},{ }^{a} b\right] \equiv\left[g,{ }^{a} b\right] \cdot\left[[g, a],{ }^{a} b\right]^{-1} \quad\left(\bmod K_{m+i+1}\right),
\end{aligned}
$$

we obtain

$$
\begin{aligned}
{ }^{\left(a K_{1}\right)}\left(\tau_{m}(g)\left(b K_{i+1}\right)\right) & ={ }^{\left(a K_{1}\right)}\left([g, b] K_{m+i+1}\right) \\
& =\left({ }^{a}[g, b]\right) K_{m+i+1} \\
& =\left(\left[g,{ }^{a} b\right] \cdot\left[[g, a],{ }^{a} b\right]^{-1}\right) K_{m+i+1} \\
& =\left(\left[g,{ }^{a} b\right] K_{m+i+1}\right)-\left(\left[[g, a],{ }^{a} b\right] K_{m+i+1}\right) \\
& =\tau_{m}(g)\left(\left({ }^{a} b\right) K_{i+1}\right)-\left[[g, a] K_{m+1},\left({ }^{a} b\right) K_{i+1}\right] \\
& =\tau_{m}(g)\left({ }^{\left(a K_{1}\right)}\left(b K_{i+1}\right)\right)-\left[\tau_{m}(g)\left(a K_{1}\right),{ }^{\left(a K_{1}\right)}\left(b K_{i+1}\right)\right],
\end{aligned}
$$

proving (6.3). Thus, we have $\tau_{m}(g) \in \operatorname{Der}_{m}\left(\bar{K}_{\bullet}\right)$.

Finally, we show that the map $\tau_{m}: G_{m} \rightarrow \operatorname{Der}_{m}\left(\bar{K}_{\bullet}\right)$ is a homomorphism. Indeed, for $g, g^{\prime} \in G_{m}, a \in K_{i}, i \geq 0$, we have

$$
\begin{aligned}
\tau_{m}\left(g g^{\prime}\right)\left(a K_{i+1}\right) & =\left[g g^{\prime}, a\right] K_{m+i+1} \\
& =\left({ }^{g}\left[g^{\prime}, a\right] \cdot[g, a]\right) K_{m+i+1} \\
& =\left(\left[g^{\prime}, a\right] \cdot[g, a]\right) K_{m+i+1} \\
& =\left[g^{\prime}, a\right] K_{m+i+1}+[g, a] K_{m+i+1} \\
& =\tau_{m}\left(g^{\prime}\right)\left(a K_{i+1}\right)+\tau_{m}(g)\left(a K_{i+1}\right)=\left(\tau_{m}(g)+\tau_{m}\left(g^{\prime}\right)\right)\left(a K_{i+1}\right) .
\end{aligned}
$$

It is easy to prove the following.

Proposition 6.3. For $m \geq 0$, we have

$$
\operatorname{ker}\left(\tau_{m}\right)=G_{m} \cap \mathcal{F}_{m+1}^{K_{*}}\left(G_{0}\right)=\left\{g \in G_{m} \mid\left[g, K_{i}\right] \subset K_{m+i+1} \text { for } i \geq 0\right\},
$$

where $\mathcal{F}_{*}^{K_{*}}\left(G_{0}\right)$ is the Johnson filtration of $G_{0}$ induced by $K_{*}$.

Set $\bar{G}_{m}=\operatorname{gr}_{m}\left(G_{*}\right)$ for each $m \geq 0$. By Propositions 6.1 and 6.2, $\tau_{m}$ induces a homomorphism

$$
\bar{\tau}_{m}: \bar{G}_{m} \longrightarrow \operatorname{Der}_{m}\left(\bar{K}_{\bullet}\right) .
$$

By Proposition 6.3, we have

$$
\operatorname{ker}\left(\bar{\tau}_{m}\right)=\left(G_{m} \cap \mathcal{F}_{m+1}^{K_{*}}\left(G_{0}\right)\right) / G_{m+1} .
$$


6.2. The Johnson morphism. In this subsection, we show that the family of all generalized Johnson homomorphisms form a morphism of eg-Lie algebras, which we call the Johnson morphism.

Theorem 6.4. Let an extended $N$-series $G_{*}$ act on an extended $N$-series $K_{*}$, and set $\bar{G}_{\bullet}=\operatorname{gr}_{\bullet}\left(G_{*}\right), \bar{K}_{\bullet}=\operatorname{gr}_{\bullet}\left(K_{*}\right)$. Then the family $\bar{\tau}_{\bullet}=\left(\bar{\tau}_{m}\right)_{m \geq 0}$ of all homomorphisms $\bar{\tau}_{m}$ defined by (6.5) is a morphism of eg-Lie algebras

$$
\bar{\tau}_{\bullet}: \bar{G}_{\bullet} \longrightarrow \operatorname{Der} \bullet\left(\bar{K}_{\bullet}\right) \text {. }
$$

Moreover, $\bar{\tau}_{\bullet}$ is injective if and only if $G_{*}$ is the Johnson filtration $\mathcal{F}_{*}^{K_{*}}\left(G_{0}\right)$.

Proof. We know that $\bar{\tau}_{m}$ is a homomorphism for each $m \geq 0$. Let us check that $\left(\bar{\tau}_{m}\right)_{m \geq 1}: \bar{G}_{+} \rightarrow \operatorname{Der}_{+}\left(\bar{K}_{\bullet}\right)$ preserves the Lie bracket. For $g \in G_{m}, g^{\prime} \in G_{n}$, $m, n \geq 1, a \in K_{i}, i \geq 0$, we have

$$
\begin{aligned}
& \bar{\tau}_{m+n}\left(\left[g K_{m+1}, g^{\prime} K_{n+1}\right]\right)\left(a K_{i+1}\right) \\
= & \bar{\tau}_{m+n}\left(\left[g, g^{\prime}\right] K_{m+n+1}\right)\left(a K_{i+1}\right) \\
= & {\left[\left[g, g^{\prime}\right], a\right] K_{m+n+i+1} } \\
= & {\left[\left[g, g^{\prime}\right],\left[a, g^{\prime}\right] \cdot{ }^{\prime} a\right] K_{m+n+i+1} } \\
= & {\left[\left[g, g^{\prime}\right],{ }^{\prime} a\right] K_{m+n+i+1} } \\
= & \left(\left[{ }^{g} g^{\prime},[a, g]\right] \cdot\left[{ }^{a} g,\left[g^{\prime}, a\right]\right]\right) K_{m+n+i+1} \\
= & \left(\left[\left[g, g^{\prime}\right] g^{\prime},[a, g]\right] \cdot\left[[a, g] g,\left[g^{\prime}, a\right]\right]\right) K_{m+n+i+1} \\
= & \left(\left[g^{\prime},[a, g]\right] \cdot\left[g,\left[g^{\prime}, a\right]\right] \cdot\left[[a, g],\left[g^{\prime}, a\right]\right]\right) K_{m+n+i+1} \\
= & \left(\left[g^{\prime},[g, a]^{-1}\right] \cdot\left[g,\left[g^{\prime}, a\right]\right] \cdot\left[[g, a]^{-1},\left[g^{\prime}, a\right]\right]\right) K_{m+n+i+1} \\
= & -\bar{\tau}_{n}\left(g^{\prime} G_{n+1}\right)\left(\bar{\tau}_{m}\left(g G_{m+1}\right)\left(a K_{i+1}\right)\right)+\bar{\tau}_{m}\left(g G_{m+1}\right)\left(\bar{\tau}_{n}\left(g^{\prime} G_{n+1}\right)\left(a K_{i+1}\right)\right) \\
& -\delta_{i, 0}\left[\bar{\tau}_{m}\left(g G_{m+1}\right)\left(a K_{i+1}\right), \bar{\tau}_{n}\left(g^{\prime} G_{n+1}\right)\left(a K_{i+1}\right)\right] \\
= & {\left[\bar{\tau}_{m}\left(g G_{m+1}\right), \bar{\tau}_{n}\left(g^{\prime} G_{n+1}\right)\right]\left(a K_{i+1}\right) . }
\end{aligned}
$$

Hence $\left(\bar{\tau}_{m}\right)_{m \geq 1}$ is a morphism of graded Lie algebras.

It remains to verify the equivariance property for $\bar{\tau}_{\bullet}$. For $g \in G_{0}, g^{\prime} \in G_{m}$, $m \geq 1, a \in K_{i}, i \geq 1$, we have

$$
\begin{aligned}
\bar{\tau}_{m}\left({ }^{\left(g G_{1}\right)}\left(g^{\prime} G_{m+1}\right)\right)\left(a K_{i+1}\right) & =\bar{\tau}_{m}\left(\left({ }^{g} g^{\prime}\right) G_{m+1}\right)\left(a K_{i+1}\right) \\
& =\left[{ }^{g} g^{\prime}, a\right] K_{m+i+1} \\
& ={ }^{g}\left[g^{\prime},{ }^{-1} a\right] K_{m+i+1} \\
& =\bar{\tau}_{0}\left(g G_{1}\right)\left(\bar{\tau}_{m}\left(g^{\prime} G_{m+1}\right)\left(\bar{\tau}_{0}\left(g G_{1}\right)^{-1}\left(a K_{i+1}\right)\right)\right) \\
& =\left({ }^{\bar{\tau}_{0}}\left(g G_{1}\right) \bar{\tau}_{m}\left(g^{\prime} G_{m+1}\right)\right)\left(a K_{i+1}\right) .
\end{aligned}
$$

Hence $\bar{\tau}_{\bullet}$ is a morphism of eg-Lie algebras.

The second statement of the theorem says that $\bar{\tau}_{m}$ is injective for all $m \geq 0$ if and only if we have $G_{m}=\mathcal{F}_{m}^{K_{*}}\left(G_{0}\right)$ for all $m \geq 0$. This equivalence is easily checked by induction on $m \geq 0$ using (6.6).

As a special case of Theorem 6.4, we obtain the following. 
Corollary 6.5. Let $K_{*}$ be an extended $N$-series. Then we have an injective morphism of eg-Lie algebras

$$
\bar{\tau}_{\bullet}: \operatorname{gr}_{\bullet}\left(\operatorname{Aut}_{*}\left(K_{*}\right)\right) \longrightarrow \operatorname{Der} \bullet\left(\operatorname{gr}_{\bullet}\left(K_{*}\right)\right)
$$

where $\operatorname{Aut}_{*}\left(K_{*}\right)$ is the Johnson filtration of $\operatorname{Aut}\left(K_{*}\right)$ defined by (3.7).

Example 6.6. Continuing Examples 3.4 and 5.4, let us consider the adjoint actions $\mathrm{Ad}^{K_{*}}$ and $\operatorname{ad}^{\mathrm{gr}}{ }^{{ }^{\prime}\left(K_{*}\right)}$. The morphism $\bar{\tau}_{\bullet}$ in (6.8) fits into the following commutative diagram:

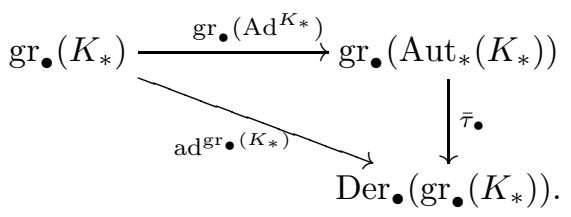

\section{Truncation of a DeRIVAtion EG-Lie ALgebra}

Here we define the "truncation" $D_{\bullet}\left(L_{\bullet}\right)$ of the derivation eg-Lie algebra Der. $\left(L_{\bullet}\right)$ of an eg-Lie algebra $L_{\bullet}$. This structure is useful mainly when the positive part $L_{+}$ of $L_{\bullet}$ is a free Lie algebra generated by its degree 1 part.

7.1. Truncation of a derivation eg-Lie algebra. Let $L_{\bullet}$ be an eg-Lie algebra. Here we define a graded group $D_{\bullet}\left(L_{\bullet}\right)=\left(D_{m}\left(L_{\bullet}\right)\right)_{m \geq 0}$, which we call the truncation of $\operatorname{Der} \bullet\left(L_{\bullet}\right)$. Set

$$
\begin{aligned}
D_{0}\left(L_{\bullet}\right)=\{ & \left(d_{0}, d_{1}\right) \in \operatorname{Aut}\left(L_{0}\right) \times \operatorname{Aut}\left(L_{1}\right) \\
& \left.\mid d_{1}\left({ }^{a} b\right)={ }^{d_{0}(a)}\left(d_{1}(b)\right) \text { for } a \in L_{0}, b \in L_{1}\right\},
\end{aligned}
$$

which is a subgroup of $\operatorname{Aut}\left(L_{0}\right) \times \operatorname{Aut}\left(L_{1}\right)$. For $m \geq 1$, define an abelian group $D_{m}\left(L_{\bullet}\right)$ by

$$
\begin{aligned}
D_{m}\left(L_{\bullet}\right)=\{ & \left(d_{0}, d_{1}\right) \in Z^{1}\left(L_{0}, L_{m}\right) \times \operatorname{Hom}\left(L_{1}, L_{m+1}\right) \\
& \left.\mid d_{1}\left({ }^{a} b\right)=\left[d_{0}(a),{ }^{a} b\right]+{ }^{a}\left(d_{1}(b)\right) \text { for } a \in L_{0}, b \in L_{1}\right\},
\end{aligned}
$$

where $Z^{1}\left(L_{0}, L_{m}\right)$ denotes the group of $L_{m}$-valued 1-cocycles on $L_{0}$ :

$$
Z^{1}\left(L_{0}, L_{m}\right)=\left\{d_{0}: L_{0} \rightarrow L_{m} \mid d_{0}(a b)=d_{0}(a)+{ }^{a}\left(d_{0}(b)\right) \text { for } a, b \in L_{0}\right\} .
$$

For every $m \geq 0$, there is a homomorphism

$$
t_{m}: \operatorname{Der}_{m}\left(L_{\bullet}\right) \longrightarrow D_{m}\left(L_{\bullet}\right), \quad\left(d_{i}\right)_{i \geq 0} \longmapsto\left(d_{0}, d_{1}\right) .
$$

Lemma 7.1. If the positive part $L_{+}$of an eg-Lie algebra $L_{\bullet}$ is generated by its degree 1 part $L_{1}$, then $t_{m}$ is injective for each $m \geq 0$.

Proof. First, we prove that the kernel of $t_{0}$ is trivial. Take $d=\left(d_{i}\right)_{i \geq 0}$ such that $\left(d_{0}, d_{1}\right)=\left(\operatorname{id}_{L_{0}}, \mathrm{id}_{L_{1}}\right)$. We prove $d_{i}=\operatorname{id}_{L_{i}}$ for all $i \geq 0$ by induction on $i \geq 0$. Let $i \geq 2$. Since $L_{1}$ generates $L_{+}, L_{i}$ is generated by the elements $[x, y]$ with $x \in L_{1}$, $y \in L_{i-1}$. We have

$$
d_{i}([x, y])=\left[d_{1}(x), d_{i-1}(y)\right]=[x, y]
$$

by the induction hypothesis. Hence $d_{i}=\mathrm{id}_{L_{i}}$.

Now we prove that the kernel of $t_{m}$ is trivial for $m \geq 1$. Take $d=\left(d_{i}\right)_{i \geq 0}$ with $\left(d_{0}, d_{1}\right)=(0,0)$. We prove $d_{i}=0$ for all $i \geq 0$ by induction on $i \geq 0$. Let $i \geq 2$. 
Since $L_{1}$ generates $L_{+}, L_{i}$ is generated by the elements $[x, y]$ with $x \in L_{1}, y \in L_{i-1}$. We have

$$
d_{i}([x, y])=\left[d_{1}(x), y\right]+\left[x, d_{i-1}(y)\right]=0
$$

by the induction hypothesis. Hence $d_{i}=0$.

Lemma 7.2. Let $L_{+}=\bigoplus_{i>1} L_{i}$ be the graded Lie algebra freely generated by an abelian group $A$ in degree 1 . For $m \geq 1$, every homomorphism $d_{1}: A=L_{1} \rightarrow L_{m+1}$ extends (uniquely) to a derivation d of $L_{+}$of degree $m$.

This lemma is well known at least for $A$ a free abelian group. (See [34, Lemma 0.7] for instance.) We give a proof here since we could not find a suitable reference for the general case.

Proof of Lemma \%.2. Let $M=\bigoplus_{i \geq 1} M_{i}$ be the non-unital, non-associative algebra freely generated by $A$ in degree 1 . (Thus we have $M_{1}=A, M_{2}=A \otimes A, M_{3}=$ $A \otimes(A \otimes A) \oplus(A \otimes A) \otimes A$, etc. $)$ Let $*: M \times M \rightarrow M$ denote the multiplication in $M$. Then the free Lie algebra $L_{+}$may be defined as the quotient $M / I$ of $M$ by the ideal $I$ generated by the elements

$$
b * b, \quad b_{1} *\left(b_{2} * b_{3}\right)+b_{2} *\left(b_{3} * b_{1}\right)+b_{3} *\left(b_{1} * b_{2}\right)
$$

for all $b, b_{1}, b_{2}, b_{3} \in M$.

Let $\tilde{d}_{1}: M_{1} \rightarrow M_{m+1}$ be a lift of $d_{1}$ to $M_{m+1}$, i.e., we require that the diagram

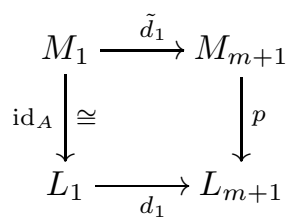

commutes, where $p$ denotes the projection. The map $\tilde{d}_{1}$ extends uniquely to a degree $m$ derivation $\tilde{d}_{+}=\left(\tilde{d}_{i}: M_{i} \rightarrow M_{m+i}\right)_{i \geq 1}$ of $M$. One easily checks $\tilde{d}_{+}(I) \subset I$. Therefore, $\tilde{d}_{+}$induces a family of homomorphisms $d_{+}=\left(d_{i}: L_{i} \rightarrow L_{m+i}\right)_{i \geq 1}$. Clearly, $d_{+}$is a degree $m$ derivation of $L_{+}$.

Proposition 7.3. If the positive part $L_{+}$of an eg-Lie algebra $L_{\bullet}$ is freely generated by its degree 1 part $L_{1}$, then $t_{m}$ is an isomorphism for all $m \geq 0$.

Proof. By Lemma 7.1, $t_{m}$ is injective. Thus it suffices to check that if $\left(d_{0}, d_{1}\right) \in$ $D_{m}\left(L_{\bullet}\right)$, then it extends to at least one $\left(d_{i}\right)_{i \geq 0} \in \operatorname{Der}_{m}\left(L_{\bullet}\right)$.

First, let $m=0$. The automorphism $d_{1}$ of $L_{1}$ extends uniquely to an automorphism $d_{+}=\left(d_{i}: L_{i} \rightarrow L_{i}\right)_{i \geq 1}$ of the graded Lie algebra $L_{+}$. It suffices to prove the equivariance property, i.e.,

$$
d_{i}\left({ }^{a} b\right)={ }^{d_{0}(a)}\left(d_{i}(b)\right)
$$

for $a \in L_{0}, b \in L_{i}, i \geq 1$, which is verified by induction on $i \geq 1$.

Now, let $m \geq 1$. By Lemma 7.2, we can extend the homomorphism $d_{1}$ to a derivation $d_{+}=\left(d_{i}: L_{i} \rightarrow L_{m+i}\right)_{i \geq 1}$ of $L_{+}$of degree $m$. It suffices to prove that

$$
d_{i}\left({ }^{a} b\right)=\left[d_{0}(a),{ }^{a} b\right]+{ }^{a}\left(d_{i}(b)\right)
$$


for $a \in L_{0}, b \in L_{i}, i \geq 1$. The proof is by induction on $i \geq 1$. Let $i \geq 2$. We may assume $b=\left[b^{\prime}, b^{\prime \prime}\right], b^{\prime} \in L_{1}, b^{\prime \prime} \in L_{i-1}$. Then we have

$$
\begin{aligned}
d_{i}\left({ }^{a} b\right) & =d_{i}\left(\left[{ }^{a} b^{\prime},{ }^{a} b^{\prime \prime}\right]\right) \\
& =\left[d_{1}\left({ }^{a} b^{\prime}\right),{ }^{a} b^{\prime \prime}\right]+\left[{ }^{a} b^{\prime}, d_{i-1}\left({ }^{a} b^{\prime \prime}\right)\right] \\
& =\left[\left[d_{0}(a),{ }^{a} b^{\prime}\right]+{ }^{a}\left(d_{1}\left(b^{\prime}\right)\right),{ }^{a} b^{\prime \prime}\right]+\left[{ }^{a} b^{\prime},\left[d_{0}(a),{ }^{a} b^{\prime \prime}\right]+{ }^{a}\left(d_{i-1}\left(b^{\prime \prime}\right)\right)\right] \\
& =\left[\left[d_{0}(a),{ }^{a} b^{\prime}\right],{ }^{a} b^{\prime \prime}\right]+\left[{ }^{a}\left(d_{1}\left(b^{\prime}\right)\right),{ }^{a} b^{\prime \prime}\right]+\left[{ }^{a} b^{\prime},\left[d_{0}(a),{ }^{a} b^{\prime \prime}\right]\right]+\left[{ }^{a} b^{\prime},{ }^{a}\left(d_{i-1}\left(b^{\prime \prime}\right)\right)\right] \\
& =\left[d_{0}(a),\left[{ }^{a} b^{\prime},{ }^{a} b^{\prime \prime}\right]\right]+{ }^{a}\left[d_{1}\left(b^{\prime}\right), b^{\prime \prime}\right]+{ }^{a}\left[b^{\prime}, d_{i-1}\left(b^{\prime \prime}\right)\right] \\
& =\left[d_{0}(a),{ }^{a}\left[b^{\prime}, b^{\prime \prime}\right]\right]+{ }^{a}\left(d_{i}\left(\left[b^{\prime}, b^{\prime \prime}\right]\right)\right)=\left[d_{0}(a),{ }^{a} b\right]+{ }^{a}\left(d_{i}(b)\right),
\end{aligned}
$$

where the third identity is given by the induction hypothesis.

7.2. The eg-Lie algebra structure of the truncation. Let $L$ • be an eg-Lie algebra whose positive part $L_{+}$is freely generated by $L_{1}$. By Proposition 7.3, $D_{\bullet}\left(L_{\bullet}\right)$ is endowed with a unique eg-Lie algebra structure such that

$$
t_{\bullet}=\left(t_{m}\right)_{m \geq 0}: \operatorname{Der}_{\bullet}\left(L_{\bullet}\right) \longrightarrow D_{\bullet}\left(L_{\bullet}\right)
$$

is an eg-Lie algebra isomorphism. The following is easily derived from the definition of Der• $\left(L_{\bullet}\right)$ given in Section 5.2.

Proposition 7.4. Let $L_{\bullet}$ be an eg-Lie algebra such that $L_{+}$is freely generated by $L_{1}$ as a graded Lie algebra. Then the graded group $D_{\bullet}\left(L_{\bullet}\right)$ has the following eg-Lie algebra structure.

(1) The Lie bracket $\left[d, d^{\prime}\right] \in D_{m+n}\left(L_{\bullet}\right)$ of $d=\left(d_{0}, d_{1}\right) \in D_{m}\left(L_{\bullet}\right)$ and $d^{\prime}=$ $\left(d_{0}^{\prime}, d_{1}^{\prime}\right) \in D_{n}\left(L_{\bullet}\right)$ with $m, n \geq 1$ is defined by

$$
\begin{array}{ll}
{\left[d, d^{\prime}\right]_{0}(a)=d_{n}\left(d_{0}^{\prime}(a)\right)-d_{m}^{\prime}\left(d_{0}(a)\right)-\left[d_{0}(a), d_{0}^{\prime}(a)\right]} & \text { for } a \in L_{0}, \\
{\left[d, d^{\prime}\right]_{1}(b)=d_{n+1}\left(d_{1}^{\prime}(b)\right)-d_{m+1}^{\prime}\left(d_{1}(b)\right)} & \text { for } b \in L_{1},
\end{array}
$$

where $d_{+}=\left(d_{i}\right)_{i \geq 1}$ and $d_{+}^{\prime}=\left(d_{j}^{\prime}\right)_{j \geq 1}$ are the derivations of $L_{+}$extending $d_{1}$ and $d_{1}^{\prime}$, respectively.

(2) The action ${ }^{f} d \in D_{m}\left(L_{\bullet}\right)$ of $f=\left(f_{0}, f_{1}\right) \in D_{0}\left(L_{\bullet}\right)$ on $d=\left(d_{0}, d_{1}\right) \in$ $D_{m}\left(L_{\bullet}\right)$ with $m \geq 1$ is defined by

$$
\begin{aligned}
& \left({ }^{f} d\right)_{0}(a)=f_{m} d_{0} f_{0}^{-1}(a) \quad \text { for } a \in L_{0}, \\
& \left({ }^{f} d\right)_{1}(b)=f_{m+1} d_{1} f_{1}^{-1}(b) \quad \text { for } b \in L_{1},
\end{aligned}
$$

where $f_{+}=\left(f_{i}\right)_{i \geq 1}$ is the automorphism of $L_{+}$extending $f_{1}$.

\section{EXTENDED N-SERIES ASSOCIATED WITH N-SERIES}

In this section, we illustrate the constructions of the previous sections with the extended N-series defined by $\mathrm{N}$-series.

8.1. Extended $\mathbf{N}$-series associated with $\mathbf{N}$-series. Let $K_{+}=\left(K_{m}\right)_{m \geq 1}$ be an N-series of a group $K=K_{1}$. We consider here the extended N-series $K_{*}=$ $\left(K_{m}\right)_{m \geq 0}$ obtained by setting $K_{0}=K_{1}=K$.

By an action of an extended N-series $G_{*}$ on $K_{+}$we mean an action of $G_{*}$ on $K_{*}$.

Let $L_{+}$be a graded Lie algebra. Let $\operatorname{Der}_{0}\left(L_{+}\right)=\operatorname{Aut}\left(L_{+}\right)$be the automorphism group of $L_{+}$and, for $m \geq 1$, let $\operatorname{Der}_{m}\left(L_{+}\right)$denote the group of derivations of $L_{+}$of 
degree $m$. We call $\operatorname{Der}_{+}\left(L_{+}\right)=\left(\operatorname{Der}_{m}\left(L_{+}\right)\right)_{m \geq 1}$ the graded Lie algebra of positivedegree derivations of $L_{+}$. The group Aut $\left(L_{+}\right)$acts on $\operatorname{Der}_{+}\left(L_{+}\right)$by conjugation. Thus Der. $\left(L_{+}\right)=\left(\operatorname{Der}_{m}\left(L_{+}\right)\right)_{m \geq 0}$ is an eg-Lie algebra.

Theorem 6.4 implies the following.

Corollary 8.1. Let an extended $N$-series $G_{*}$ act on an $N$-series $K_{+}$, and let $\bar{G}_{\bullet}=$ $\operatorname{gr}\left(G_{*}\right), \bar{K}_{+}=\operatorname{gr}_{+}\left(K_{+}\right)$. Then the family $\bar{\tau}_{\bullet}=\left(\bar{\tau}_{m}\right)_{m \geq 0}$ of all homomorphisms $\bar{\tau}_{m}$ defined by (6.5) is a morphism of eg-Lie algebras

$$
\bar{\tau}_{\bullet}: \bar{G}_{\bullet} \longrightarrow \operatorname{Der} \bullet\left(\bar{K}_{+}\right) .
$$

Moreover, $\bar{\tau}_{\bullet}$ is injective if and only if $G_{*}$ is the Johnson filtration $\mathcal{F}_{*}^{K_{*}}\left(G_{0}\right)$.

In the rest of this section, we consider $N$-series with special properties (called $N_{0}$-series and $N_{p}$-series). We show that if a group $G$ acts on such a special N-series, then the positive part of the Johnson filtration of $G$ is an N-series of the same kind.

8.2. $\mathbf{N}_{0}$-series. An $N_{0}$-series of a group $K$ is an $\mathrm{N}$-series $K_{+}$such that $K / K_{m}$ is torsion-free for all $m \geq 1$.

An $\mathrm{N}$-series $K_{+}$can be transformed into an $\mathrm{N}_{0}$-series $\sqrt{K_{+}}$by considering the root sets of its successive terms. Specifically, we define for all $m \geq 1$

$$
\sqrt{K_{m}}=\left\{x \in K \mid x^{i} \in K_{m} \text { for some } i \geq 1\right\} .
$$

See [29, §IV.1.3] or [30, §11, Lemma 1.8] in the case of the lower central series, and [21, Lemma 4.4] in the general case. Note that $\sqrt{K_{+}}$is the smallest $\mathrm{N}_{0}$-series of $K$ containing $K_{+}$: thus, $\sqrt{K}_{+}=K_{+}$if and only if $K_{+}$is an $\mathrm{N}_{0}$-series.

Example 8.2. The rational lower central series of a group $K$ is the $\mathrm{N}_{0}$-series $\sqrt{\Gamma_{+} K}=\left(\sqrt{\Gamma_{m} K}\right)_{m \geq 1}$ associated to the lower central series $\Gamma_{+} K$ of $K$. It is the smallest $\mathrm{N}_{0}$-series of $K$.

Proposition 8.3. Let a group $G$ act on an $N_{0}$-series $K_{+}$. Then the positive part $\mathcal{F}_{+}^{K_{*}}(G)$ of the Johnson filtration $\mathcal{F}_{*}^{K_{*}}(G)$ is an $N_{0}$-series.

Proof. Set $G_{*}=\mathcal{F}_{*}^{K_{*}}(G)$. By Proposition 3.1, $G_{+}$is an N-series of $G_{1}$. Therefore, it remains to show that $G_{m} / G_{m+1}$ is torsion-free for $m \geq 1$. By Corollary 8.1, the $m$ th Johnson homomorphism induces an injection

$$
\bar{\tau}_{m}: G_{m} / G_{m+1} \longrightarrow \operatorname{Der}_{m}\left(\bar{K}_{+}\right) .
$$

Hence it suffices to check that $\operatorname{Der}_{m}\left(\bar{K}_{+}\right)$is torsion-free. This follows since $\bar{K}_{+}$ itself is torsion-free.

8.3. $\mathbf{N}_{p}$-series. Let $p$ be a prime. An $N_{p}$-series of a group $K$ is an $\mathrm{N}$-series $K_{+}$ such that $\left(K_{m}\right)^{p} \subset K_{m p}$ for all $m \geq 1$. By a result of Lazard [16, Corollary 6.8], $\bar{K}_{+}=\bigoplus_{i \geq 1} K_{i} / K_{i+1}$ is a restricted Lie algebra over the field $\mathbb{F}_{p}=\mathbb{Z} / p \mathbb{Z}$, whose p-operation

$$
(\cdot)^{[p]}: \bar{K}_{+} \longrightarrow \bar{K}_{+}
$$

is defined by $\left(x K_{i+1}\right)^{[p]}=\left(x^{p} K_{i p+1}\right)$ for $x \in K_{i}, i \geq 1$.

Every N-series $K_{+}$can be transformed into an $\mathrm{N}_{p}$-series $K_{+}^{[p]}$ defined by

$$
K_{m}^{[p]}=\prod_{i \geq 1, j \geq 0, i p^{j} \geq m} K_{i}^{p^{j}} \quad \text { for } m \geq 1 .
$$


See $[29, \S I V .1 .22]$ or $[30, \S 11$, Lemma 1.18] in the case of the lower central series, and [21, Lemma 4.6] in the general case. Note that $K_{+}^{[p]}$ is the smallest $\mathrm{N}_{p}$-series of $K$ containing $K_{+}$: thus, $K_{+}^{[p]}=K_{+}$if and only if $K_{+}$is an $\mathrm{N}_{p}$-series.

Example 8.4. The Zassenhaus mod-p lower central series (also called the Zassenhaus filtration) of a group $K$ [41] is the $\mathrm{N}_{p}$-series $\Gamma_{+}^{[p]} K$ associated to the lower central series $\Gamma_{+} K$ of $K$ :

$$
\Gamma_{m}^{[p]} K=\prod_{i \geq 1, j \geq 0, i p^{j} \geq m}\left(\Gamma_{i} K\right)^{p^{j}} \quad \text { for } m \geq 1 .
$$

This "mod- $p$ " variant of $\Gamma_{+} K$ should not be confused with the Stallings mod-p lower central series (also called the lower exponent-p central series) $\Gamma_{+}^{\langle p\rangle} K$ [38], which is defined inductively by $\Gamma_{1}^{\langle p\rangle} K=K$ and

$$
\Gamma_{m+1}^{\langle p\rangle} K=\left(\Gamma_{m}^{\langle p\rangle} K\right)^{p}\left[K, \Gamma_{m}^{\langle p\rangle} K\right] \quad \text { for } m \geq 1 .
$$

Indeed $\Gamma_{+}^{[p]} K$ is the smallest $\mathrm{N}_{p}$-series of $K$, whereas $\Gamma_{+}^{\langle p\rangle} K$ is the smallest N-series $K_{+}$of $K$ such that $\left(K_{m}\right)^{p} \subset K_{m+1}$ for $m \geq 1$.

Proposition 8.5. Let a group $G$ act on an $N_{p}$-series $K_{+}$. Then the positive part $\mathcal{F}_{+}^{K_{*}}(G)$ of the Johnson filtration $\mathcal{F}_{*}^{K_{*}}(G)$ is an $N_{p}$-series.

Proof. Set $G_{*}=\mathcal{F}_{*}^{K_{*}}(G)$. Since $G_{+}$is an N-series of $G_{1}$ by Proposition 3.1, it suffices to check $\left(G_{m}\right)^{p} \subset G_{m p}$ for all $m \geq 1$. Let $g \in G_{m}$ and $x \in K_{j}, j \geq 1$. By Dark's commutator formula (see [30, 111 , Theorem 1.16]), we have

$$
\left[g^{p}, x\right]=\prod_{i=1}^{p} c_{i}^{\left(\begin{array}{c}
p \\
i
\end{array}\right)},
$$

where $c_{i}$ is a product of iterated commutators, each with at least $i$ components equal to $g^{ \pm 1}$ and at least one component equal to $x^{ \pm 1}$. It follows that

$$
c_{i} \in K_{j+i m}, \quad \text { for } i=1, \ldots, p \text {. }
$$

Therefore, $c_{p} \in K_{j+p m}$ and, for $i \in\{1, \ldots, p-1\}$, we have

$$
c_{i}^{\left(\begin{array}{c}
p \\
i
\end{array}\right)} \in\left(K_{j+i m}\right)^{p} \subset K_{j p+i m p} \subset K_{j+m p}
$$

since $p$ divides $\left(\begin{array}{c}p \\ i\end{array}\right)$ and $K_{+}$is an $\mathrm{N}_{p^{-}}$-series. Hence $\left[g^{p}, x\right] \in K_{j+m p}$ and $g^{p} \in G_{m p}$.

Remark 8.6. Let a group $G$ act on an $\mathrm{N}_{p}$-series $K_{+}$. Since $\bar{K}_{+}$is a restricted Lie algebra over $\mathbb{F}_{p}$, so is $\operatorname{Der}_{+}\left(\bar{K}_{+}\right)$with $p$-operation defined by the $p$-th power. Besides, by Proposition 8.5, $\operatorname{gr}_{+} \mathcal{F}_{+}^{K_{*}}(G)$ is a restricted Lie algebra over $\mathbb{F}_{p}$. One can expect that the positive part of the Johnson morphism in Corollary 8.1,

$$
\bar{\tau}_{+}: \operatorname{gr}_{+} \mathcal{F}_{+}^{K_{*}}(G) \longrightarrow \operatorname{Der}_{+}\left(\bar{K}_{+}\right),
$$

is a morphism of restricted Lie algebras (i.e., it preserves the $p$-operations). Furthermore, it is plausible that $\bar{\tau}_{+}$takes values in the restricted Lie subalgebra of $\operatorname{Der}_{+}\left(\bar{K}_{+}\right)$consisting of restricted derivations in the sense of Jacobson [11].

In degree 0 , it is easily verified that $\bar{\tau}_{0}: \mathcal{F}_{0}^{K_{*}}(G) / \mathcal{F}_{1}^{K_{*}}(G) \rightarrow \operatorname{Aut}\left(\bar{K}_{+}\right)$takes values in the subgroup of automorphisms of the restricted Lie algebra $\bar{K}_{+}$. 
Remark 8.7. An exponent-p $N$-series of a group $K$ is an N-series $K_{+}$of $K$ such that $\left(K_{m}\right)^{p} \leq K_{m+1}$ for all $m \geq 1$. For instance, the Stallings mod- $p$ lower central series $\Gamma_{+}^{\langle p\rangle} K$ of $K$ satisfies this property. We have the following variant of Proposition 8.5: If a group $G$ acts on an exponent-p $N$-series $K_{+}$, then the positive part $\mathcal{F}_{+}^{K_{*}}(G)$ of the Johnson filtration $\mathcal{F}_{*}^{K_{*}}(G)$ is an exponent-p $N$-series. The proof is easy and left to the reader.

\section{THE LOWER CENTRAL SERIES AND ITS VARIANTS}

In this section, we consider the lower central series $\Gamma_{+} K$ of a group $K$ and its variants: the rational lower central series $\sqrt{\Gamma_{+} K}$, the Zassenhaus mod- $p$ lower central series $\Gamma_{+}^{[p]} K$ and the Stallings mod- $p$ lower central series $\Gamma_{+}^{\langle p\rangle} K$.

9.1. The filtration $G_{*}^{1}$. Let $K_{+}$be an N-series of a group $K$, and extend it to an extended N-series $K_{*}$ with $K_{0}=K$. Let a group $G$ act on $K_{*}$, and let $G_{*}=$ $\mathcal{F}_{*}^{K *}(G)$ be the Johnson filtration of $G$ induced by $K_{*}$. Define a descending series $G_{*}^{1}=\left(G_{m}^{1}\right)_{m \geq 0}$ of $G$ by

$$
G_{m}^{1}=\left\{g \in G \mid[g, K] \subset K_{m+1}\right\}=\operatorname{ker}\left(G \rightarrow \operatorname{Aut}\left(K / K_{m+1}\right)\right) .
$$

Clearly, $G_{m}^{1} \geq G_{m}$ for $m \geq 0$, and $G_{0}^{1}=G=G_{0}$.

The filtration $G_{*}^{1}$ is not an extended N-series in general, but it is so for the lower central series and its variants. In fact, Andreadakis was the first to study the filtration $G_{*}^{1}$ in the case of $K_{+}=\Gamma_{+} K$ with $G=\operatorname{Aut}(K)$, and he proved the following proposition in this case [1, Theorem 1.1.(i)]. See also [6, Lemma 3.7] and [28, proof of Theorem 2.4] for $K_{+}=\Gamma_{+}^{\langle p\rangle} K$, and see [23, Lemma 2.2.4] for $K_{+}=\Gamma_{+}^{[p]} K$.

Proposition 9.1. If $K_{+}$is one of $\Gamma_{+} K, \sqrt{\Gamma_{+} K}, \Gamma_{+}^{[p]} K$ and $\Gamma_{+}^{\langle p\rangle} K$, then we have $G_{*}=G_{*}^{1}$. (In particular, $G_{*}^{1}$ is an extended $N$-series.)

Proof. To prove Proposition 9.1, it suffices to check $G_{m}^{1} \leq G_{m}=\mathcal{F}_{m}^{K_{*}}(G)$ for $m \geq 1$. Thus, we need to check

$$
\left[G_{m}^{1}, K_{n}\right] \leq K_{m+n} \text { for } m \geq 1, n \geq 2 .
$$

We prove (9.2) in the four cases separately.

Case 1: $K_{+}=\Gamma_{+} K$. Here we repeat Andreadakis' proof. We verify (9.2) by induction on $n$ as follows:

$$
\begin{aligned}
{\left[G_{m}^{1}, K_{n}\right] } & =\left[G_{m}^{1},\left[K, K_{n-1}\right]\right] \\
& \leq\left\langle\left[\left[G_{m}^{1}, K\right], K_{n-1}\right] \cdot\left[\left[G_{m}^{1}, K_{n-1}\right], K\right]\right\rangle_{K \rtimes G} \quad \text { (by Lemma 2.1) } \\
& \leq\left\langle\left\langle\left[K_{m+1}, K_{n-1}\right] \cdot\left[K_{m+n-1}, K\right]\right\rangle_{K \rtimes G} \quad\right. \text { (by the induction hypothesis) } \\
& \leq\left\langle\left\langle K_{m+n}\right\rangle_{K \rtimes G}=K_{m+n} .\right.
\end{aligned}
$$

Case 2: $K_{+}=\sqrt{\Gamma_{+} K}$. By induction on $n$, we will prove that $[g, a] \in K_{m+n}$ for $g \in G_{m}^{1}$ and $a \in K_{n}$. We have $a^{t} \in \Gamma_{n} K$ for some $t \geq 1$. We have

$$
[g, a]^{t} \underset{\left(\bmod K_{m+n}\right)}{\equiv} \prod_{i=1}^{t} a^{i-1}[g, a]=\left[g, a^{t}\right] \in\left[G_{m}^{1}, \Gamma_{n} K\right] \leq\left[G_{m}^{1},\left[K, K_{n-1}\right]\right]
$$


where $\equiv$ follows from

$$
\left[a^{i-1},[g, a]\right] \in\left[K_{n},\left[G_{m}^{1}, K\right]\right] \leq\left[K_{n}, K_{m+1}\right] \leq K_{m+n+1} \leq K_{m+n} .
$$

Similarly to Case 1 , we obtain $\left[G_{m}^{1},\left[K, K_{n-1}\right]\right] \leq K_{m+n}$ using the induction hypothesis. Therefore, we have $[g, a]^{t} \in K_{m+n}$, hence $[g, a] \in K_{m+n}$.

Case 3: $K_{+}=\Gamma_{+}^{[p]} K$. By (8.2), it suffices to prove by induction on $n$ that $\left[g, z^{p^{j}}\right] \in$ $K_{m+n}$ if $g \in G_{m}^{1}, z \in \Gamma_{i} K, i \geq 1, j \geq 0$ and $i p^{j} \geq n$.

If $j=0$, then $z \in \Gamma_{i} K \leq \Gamma_{n} K=\left[K, \Gamma_{n-1} K\right] \leq\left[K, K_{n-1}\right]$. Then we proceed as in Case 1 using the induction hypothesis.

Let $j \geq 1$. By Dark's commutator formula (see [30, $\S 11$, Theorem 1.16]), we have

$$
\left[g, z^{p^{j}}\right]=\prod_{d=1}^{p^{j}} c_{d}^{\left(p_{d}^{j}\right)},
$$

where $c_{d}$ is a product of iterated commutators, each with at least $d$ components equal to $z^{ \pm 1}$ and at least one component equal to $g^{ \pm 1}$. We can assume without loss of generality that $i$ is the least integer greater than or equal to $n / p^{j}$, so that $i<n$. By $z \in \Gamma_{i} K \leq K_{i}$ and the induction hypothesis, we have $\left[g^{ \pm 1}, z^{ \pm 1}\right] \in K_{m+i}$. It follows that

$$
c_{d} \in K_{m+d i} .
$$

For each $k \geq 1$, let $|k|_{p}$ denote the $p$-part of $k$, which is the unique power of $p$ such that $k /|k|_{p}$ is an integer coprime to $p$. Then we have $\left|\left(\begin{array}{c}p^{j} \\ d\end{array}\right)\right|_{p} \geq \frac{p^{j}}{|d|_{p}}$ (see, e.g., the proof of [30, $\S 11$, Lemma 1.18]). Therefore,

$$
\left|\left(\begin{array}{c}
p^{j} \\
d
\end{array}\right)\right|_{p}(m+d i) \geq \frac{p^{j}}{d}(m+d i) \geq \frac{p^{j}}{d} m+p^{j} i \geq m+n .
$$

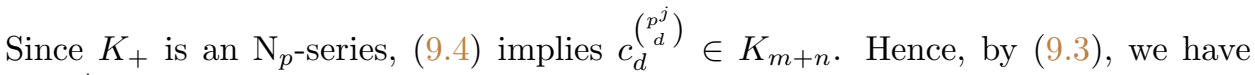
$\left[g, z^{p^{j}}\right] \in K_{m+n}$.

Case 4: $K_{+}=\Gamma_{+}^{\langle p\rangle} K$. By (8.3), it suffices to prove by induction on $n$ that we have $\left[G_{m}^{1},\left[K, K_{n-1}\right]\right] \subset K_{m+n}$ and $\left[G_{m}^{1},\left(K_{n-1}\right)^{p}\right] \subset K_{m+n}$. The former is proved similarly to Case 1 by using the induction hypothesis; to prove the latter, we will verify $\left[g, z^{p}\right] \in K_{m+n}$ for $g \in G_{m}^{1}$ and $z \in K_{n-1}$. We have

$$
\left[g, z^{p}\right]=\prod_{i=1}^{p} z^{i-1}[g, z] \underset{\left(\bmod K_{m+n}\right)}{\equiv}[g, z]^{p} \in\left[G_{m}^{1}, K_{n-1}\right]^{p} \leq\left(K_{m+n-1}\right)^{p} \leq K_{m+n},
$$

where $\equiv$ follows from

$$
\left[z^{i-1},[g, z]\right] \in\left[K_{n-1},\left[G_{m}^{1}, K_{n-1}\right]\right] \leq\left[K_{n-1}, K_{m+n-1}\right] \leq K_{m+2 n-2} \leq K_{m+n} .
$$

Hence $\left[g, z^{p}\right] \in K_{m+n}$.

This completes the proof of Proposition 9.1.

We now observe that the Johnson filtration $G_{*}^{1}=G_{*}$ can be given a ring-theoretic description, in the case of the rational (resp. Zassenhaus mod- $p$ ) lower central series. 
Corollary 9.2. If $K_{+}=\sqrt{\Gamma_{+} K}$, then for $m \geq 0$ we have

$$
G_{m}=G_{m}^{1}=\operatorname{ker}\left(\operatorname{Aut}(K) \rightarrow \operatorname{Aut}\left(\mathbb{Q}[K] / I^{m+1}\right)\right),
$$

where $I=\operatorname{ker}(\epsilon: \mathbb{Q}[K] \rightarrow \mathbb{Q})$ is the augmentation ideal.

Proof. This follows from a classical result of Malcev, Jennings and P. Hall, which computes the "dimension subgroups" with coefficients in $\mathbb{Q}$ :

$$
\left(1+I^{m+1}\right) \cap K=\sqrt{\Gamma_{m+1} K} \subset \mathbb{Q}[K] \text { for } m \geq 0 .
$$

(See, e.g., [29, §IV.1.5] or [30, §11, Theorem 1.10].)

Corollary 9.3. If $K_{+}=\Gamma_{+}^{[p]} K$, then for $m \geq 0$ we have

$$
G_{m}=G_{m}^{1}=\operatorname{ker}\left(G \rightarrow \operatorname{Aut}\left(\mathbb{F}_{p}[K] / I^{m+1}\right)\right),
$$

where $I=\operatorname{ker}\left(\epsilon: \mathbb{F}_{p}[K] \rightarrow \mathbb{F}_{p}\right)$ is the augmentation ideal.

Proof. This follows from a classical result of Jennings and Lazard, which computes the "dimension subgroups" with coefficients in $\mathbb{F}_{p}$ :

$$
\left(1+I^{m+1}\right) \cap K=\Gamma_{m+1}^{[p]} K \subset \mathbb{F}_{p}[K] \text { for } m \geq 0 .
$$

(See, e.g., [29, §IV.2.8] or [30, §11, Theorem 1.20].)

9.2. Examples and remarks. In the light of Proposition 9.1, we now relate the results and constructions of the previous sections to those in the literature.

Example 9.4. Andreadakis [1] mainly considered the case where $K_{+}=\Gamma_{+} K$ is the lower central series of a free group $K$ and $G=\operatorname{Aut}(K)$. (By Lemma 3.3, $G$ acts on $K_{+}$.) In this case, the Johnson filtration $\operatorname{Aut}_{*}\left(K_{*}\right)=G_{*}=G_{*}^{1}$ is usually called the Andreadakis-Johnson filtration. Note that $\bar{K}_{+}$is the free Lie algebra $\operatorname{Lie}\left(K^{\mathrm{ab}}\right)$ on the abelianization $K^{\mathrm{ab}}=K / \Gamma_{2} K$. Hence, by Proposition 7.3, the eg-Lie algebra morphism (7.7)

$$
t_{\bullet}: \operatorname{Der}_{\bullet}\left(\bar{K}_{+}\right) \longrightarrow D_{\bullet}\left(\bar{K}_{+}\right)
$$

is an isomorphism, where $D_{\bullet}\left(\bar{K}_{+}\right)=\left(D_{m}\left(\bar{K}_{+}\right)\right)_{m \geq 0}$ is given by

$$
D_{0}\left(\bar{K}_{+}\right)=\operatorname{Aut}\left(K^{\mathrm{ab}}\right) \quad \text { and } \quad D_{m}\left(\bar{K}_{+}\right)=\operatorname{Hom}\left(K^{\mathrm{ab}}, \operatorname{Lie}_{m+1}\left(K^{\mathrm{ab}}\right)\right)
$$

and has the eg-Lie algebra structure described in Proposition 7.4. For a finitely generated free group $K$, the composition

$$
t_{\bullet} \bar{\tau}_{\bullet}: \operatorname{gr}_{\bullet}\left(G_{*}\right) \longrightarrow D_{\bullet}\left(\bar{K}_{+}\right)
$$

has been extensively studied since Andreadakis' work; we refer to [37] for a survey.

Example 9.5. Let $\Sigma_{g, 1}$ be a compact, connected, oriented surface of genus $g$ with one boundary component, and let $K=\pi_{1}\left(\Sigma_{g, 1}, \star\right)$, where $\star \in \partial \Sigma_{g, 1}$. The mapping class group

$$
G=\operatorname{MCG}\left(\Sigma_{g, 1}, \partial \Sigma_{g, 1}\right)
$$

of $\Sigma_{g, 1}$ relative to $\partial \Sigma_{g, 1}$ acts on $K_{+}=\Gamma_{+} K$ in the natural way. By Proposition 9.1, the Johnson filtration $G_{*}$ in our sense coincides with the Johnson filtration $G_{*}^{1}$ in the usual sense, and its first term $G_{1}=G_{1}^{1}=\operatorname{ker}\left(G \rightarrow \operatorname{Aut}\left(H_{1}\left(\Sigma_{g, 1} ; \mathbb{Z}\right)\right)\right)$ is known as the Torelli group. By Example 9.4, we have an injective morphism of eg-Lie algebras $t_{\bullet} \bar{\tau}_{\bullet}: \operatorname{gr}_{\bullet}\left(G_{*}\right) \rightarrow D_{\bullet}\left(\bar{K}_{+}\right)$. The components

$$
t_{m} \tau_{m}: G_{m} \longrightarrow \operatorname{Hom}\left(H, \operatorname{Lie}_{m+1}(H)\right)
$$


for $m \geq 1$, where $H=H_{1}\left(\Sigma_{g, 1} ; \mathbb{Z}\right)$, are the original Johnson homomorphisms introduced by Johnson [12, 13] and Morita [24]. See [36] for a survey.

Remark 9.6. (i) Since the rational lower central series of a free group coincides with the lower central series, we could replace the latter by the former in Examples 9.4 and 9.5. Thus, Corollary 9.2 implies that the Johnson filtration of the mapping class group of $\Sigma_{g, 1}$ (resp. the Andreadakis-Johnson filtration of the automorphism group of a free group) can be described using Fox's free differential calculus [24, 31].

(ii) Example 9.5 can be adapted to a closed oriented surface $\Sigma_{g}$ of genus $g$. In this case, additional technicalities arise since $K=\pi_{1}\left(\Sigma_{g}\right)$ is not free, and the mapping classes of $\Sigma_{g}$ act on $K$ as outer automorphisms. The Johnson homomorphisms in this case were introduced by Morita [25].

Example 9.7. As in Example 9.5, we consider the mapping class group $G=$ $\operatorname{MCG}\left(\Sigma_{g, 1}, \partial \Sigma_{g, 1}\right)$ acting on $K=\pi_{1}\left(\Sigma_{g, 1}, \star\right)$. Here, let $K_{+}$be one of the two versions of the mod- $p$ lower central series. Note that the associated graded Lie algebra $\bar{K}_{+}$is defined over $\mathbb{F}_{p}$ in both cases. If $K_{+}=\Gamma_{+}^{[p]} K\left(\operatorname{resp} . \Gamma_{+}^{\langle p\rangle} K\right)$, then the Johnson filtration $G_{*}$ induced by $K_{*}$ coincides with the "Zassenhaus (resp. Stallings) mod- $p$ Johnson filtration" considered by Cooper in [6], and its first term $G_{1}=\operatorname{ker}\left(G \rightarrow \operatorname{Aut}\left(H_{1}\left(\Sigma_{g, 1} ; \mathbb{F}_{p}\right)\right)\right)$ is the mod-p Torelli group. Furthermore, the " $m$ th Zassenhaus (resp. Stallings) mod- $p$ Johnson homomorphism" for $m \geq 1$ defined in [6] coincides with the composition

$$
G_{m} \stackrel{\tau_{m}}{\longrightarrow} \operatorname{Der}_{m}\left(\bar{K}_{+}\right) \stackrel{t_{m}}{\longrightarrow} \operatorname{Hom}\left(\bar{K}_{1}, \bar{K}_{m+1}\right) .
$$

According to Proposition 9.1, we have $\operatorname{ker}\left(t_{m} \tau_{m}\right)=G_{m+1}$. In fact, these constructions for $K_{+}=\Gamma_{+}^{\langle p\rangle} K$ had been used by Paris [28] to prove that the mod- $p$ Torelli group (of an arbitrary compact, oriented surface) is residually a $p$-group.

Now let us focus on the case $K_{+}=\Gamma_{+}^{[p]} K$. In this case, the graded Lie algebras $\bar{K}_{+}, \operatorname{Der}_{+}\left(\bar{K}_{+}\right)$and $\bar{G}_{+}$are restricted over $\mathbb{F}_{p}$. (See Proposition 8.5 and Remark 8.6.) Since $K$ is a free group, $\bar{K}_{+}$is the free restricted Lie algebra over $\mathbb{F}_{p}$ generated by $\bar{K}_{1} \simeq H_{1}\left(\Sigma_{g, 1} ; \mathbb{F}_{p}\right)[16$, Theorem 6.5]. By Corollary 9.3, we can describe $G_{*}$ using Fox's free differential calculus, so that $G_{*}$ coincides with Perron's "modulo $p$ Johnson filtration" [32]. (See also [6, Theorem 4.7] in this connection.) By Proposition 8.5, this filtration satisfies $\left(G_{m}\right)^{p} \subset G_{m p}$ for $m \geq 1$; this fact does not seem to have been observed before.

Remark 9.8. It seems plausible that one can adapt the constructions of this paper to the setting of (extended) N-series of profinite groups and, in particular, pro- $p$ groups. In fact, the literature offers several such constructions for the lower central series of a pro $p$-group, or its variants. For instance, Asada and Kaneko [2] introduced analogues of the Johnson homomorphisms on the automorphism group of the pro- $p$ completion of a surface group. More recently, Morishita and Terashima [23] studied the Johnson homomorphisms for the automorphism group of the Zassenhaus filtration of a finitely generated pro- $p$ groups, which may be regarded as variants of Cooper's "Zassenhaus mod-p Johnson homomorphisms".

\section{Two TYPES OF SERIES ASSOCIATED WITH PAIRS OF GROUPS}

In this section, we consider two types of series $K_{*}=\left(K_{m}\right)_{m \geq 0}$ determined by their first few terms: the smallest extended N-series with given $K_{0}$ and $K_{1}$, and the smallest N-series with given $K_{0}=K_{1}$ and $K_{2}$. 
10.1. Extended $\mathrm{N}$-series determined by $K_{0}$ and $K_{1}$. Let $K=K_{0}$ be a group, and let $K_{1} \triangleleft K$. Define an extended N-series $K_{*}=\left(K_{m}\right)_{m \geq 0}$ by

$$
K_{m}= \begin{cases}K & \text { if } m=0, \\ \Gamma_{m} K_{1} & \text { if } m \geq 1 .\end{cases}
$$

Note that $K_{*}$ is the smallest extended N-series with these $K_{0}$ and $K_{1}$. The eg-Lie algebra $\bar{K}_{\bullet}=\operatorname{gr}\left(K_{*}\right)$ associated to $K_{*}$ is given by

$$
\bar{K}_{0}=K_{0} / K_{1} \quad \text { and } \quad \bar{K}_{m}=\Gamma_{m} K_{1} / \Gamma_{m+1} K_{1} \quad \text { for } m \geq 1 \text {. }
$$

Let a group $G$ act on $K$ in such a way that ${ }^{G} K_{1}=K_{1}$. Since $K_{i}=\Gamma_{i} K_{1}$ is characteristic in $K_{1}$ for all $i \geq 1, G$ acts on the extended N-series $K_{*}$ (see Lemma 3.3). Define three descending series $G_{*}^{0}, G_{*}^{1}$ and $G_{*}$ of $G$ by

$$
\begin{aligned}
& G_{m}^{0}=\left\{g \in G \mid\left[g, K_{0}\right] \subset K_{m}\right\}=\operatorname{ker}\left(G \rightarrow \operatorname{Aut}\left(K_{0} / K_{m}\right)\right), \\
& G_{m}^{1}=\left\{g \in G \mid\left[g, K_{1}\right] \subset K_{m+1}\right\}=\operatorname{ker}\left(G \rightarrow \operatorname{Aut}\left(K_{1} / K_{m+1}\right)\right), \\
& G_{m}=G_{m}^{0} \cap G_{m}^{1}=\left\{g \in G \mid\left[g, K_{0}\right] \subset K_{m},\left[g, K_{1}\right] \subset K_{m+1}\right\} .
\end{aligned}
$$

The Johnson filtration $\mathcal{F}_{*}^{K_{*}}(G)$ has the following simpler description.

Proposition 10.1. We have $G_{*}=\mathcal{F}_{*}^{K_{*}}(G)$. (Hence $G_{*}$ is an extended $N$-series.) Moreover, $G_{*}^{1}$ is an extended $N$-series.

Proof. By Proposition 9.1, we have

$$
G_{m}^{1}=\left\{g \in G \mid\left[g, K_{n}\right] \subset K_{m+n} \text { for } n \geq 1\right\}
$$

for $m \geq 0$, and $G_{*}^{1}$ is an extended N-series. By (10.2), we have

$$
G_{m}=G_{m}^{0} \cap G_{m}^{1}=\mathcal{F}_{m}^{K_{*}}(G)
$$

for $m \geq 0$.

Since $G_{m}^{1} \geq G_{m+1}^{0}$ for $m \geq 0$, the filtrations $G_{*}$ and $G_{*}^{0}$ are nested:

$$
G=G_{0}^{0}=G_{0} \geq G_{1}^{0} \geq G_{1} \geq \cdots \geq G_{m-1} \geq G_{m}^{0} \geq G_{m} \geq \cdots .
$$

Theorem 10.2. If $K_{1}$ is a non-abelian free group, then, for each $m \geq 0$, we have

$$
G_{m}=G_{m}^{1} \leq G_{m}^{0} .
$$

Proof. Note that $G_{m}^{1} \leq G_{m}^{0}$ implies $G_{m}=G_{m}^{1}$. Hence it suffices to prove by induction on $m \geq 0$ that if $g \in G,\left[g, K_{1}\right] \subset K_{m+1}$, then $\left[g, K_{0}\right] \subset K_{m}$. The case $m=0$ is trivial; let $m \geq 1$. Let $y \in K_{0}$. By the induction hypothesis, we have $[g, y] \in\left[g, K_{0}\right] \subset K_{m-1}$, i.e., $g(y)=z y$ for some $z \in K_{m-1}$. For each $x \in K_{1}$, we have

$$
{ }^{y} x \equiv g\left({ }^{y} x\right)={ }^{g(y)} g(x) \equiv{ }^{g(y)} x={ }^{z y} x=\left[z,{ }^{y} x\right]{ }^{y} x \quad\left(\bmod K_{m+1}\right),
$$

where each $\equiv$ follows from $\left[g, K_{1}\right] \subset K_{m+1}$. Therefore $\left[z, K_{1}\right] \subset K_{m+1}$. By Lemma 10.3 below, we have $z \in K_{m}$ and hence $[g, y] \in K_{m}$.

Lemma 10.3. If $F$ is a non-abelian free group and $m \geq 1$, then we have

$$
\left\{a \in F \mid[a, F] \subset \Gamma_{m+1} F\right\}=\Gamma_{m} F .
$$


Proof. Let $L_{m}=\left\{a \in F \mid[a, F] \subset \Gamma_{m+1} F\right\}$. We will prove $L_{m}=\Gamma_{m} F$ for $m \geq 1$ by induction. Let $m \geq 2$. Clearly, $\Gamma_{m} F \leq L_{m}$. By the induction hypothesis, we have $L_{m} \leq L_{m-1} \leq \Gamma_{m-1} F$. The quotient group $L_{m} / \Gamma_{m} F$, regarded as a subgroup of

$$
\Gamma_{m-1} F / \Gamma_{m} F \simeq \operatorname{Lie}_{m-1}\left(F^{\mathrm{ab}}\right), \quad \text { where } F^{\mathrm{ab}}=F / \Gamma_{2} F,
$$

is the centralizer of $\operatorname{Lie}_{1}\left(F^{\mathrm{ab}}\right)=F^{\mathrm{ab}}$ in the free Lie algebra $\operatorname{Lie}\left(F^{\mathrm{ab}}\right)$. Since $\operatorname{rank}\left(F^{\mathrm{ab}}\right) \geq 2$, the center of $\operatorname{Lie}\left(F^{\mathrm{ab}}\right)$ is trivial. Hence $L_{m} / \Gamma_{m} F$ is trivial.

Remark 10.4. Lemma 10.3 can be restated as follows. Let $F$ be a non-abelian free group, let $F_{+}=\Gamma_{+} F$ be its lower central series, and extend $F_{+}$to an extended N-series $F_{*}$ with $F_{0}=F_{1}$. Then, letting $F$ act on $F_{*}$ by conjugation, the Johnson filtration of $F$ induced by $F_{*}$ coincides with $F_{*}$.

In what follows, let $K_{1}$ be a non-abelian free group. Then $\bar{K}_{+}=\left(K_{m} / K_{m+1}\right)_{m \geq 1}$ is the free Lie algebra on $\bar{K}_{1}=K_{1}^{\mathrm{ab}}$. By Theorem 6.4 and Proposition 7.4, we obtain an injective eg-Lie algebra morphism

$$
\bar{G}_{\bullet} \stackrel{\bar{\tau}_{\bullet}}{\longrightarrow} \operatorname{Der}\left(\bar{K}_{\bullet}\right) \stackrel{t \bullet}{\simeq} D \bullet\left(\bar{K}_{\bullet}\right),
$$

where $\bar{G}_{\bullet}=\left(G_{m} / G_{m+1}\right)_{m \geq 0}$. By (7.1) and (7.2), the $m$ th Johnson homomorphism $t_{m} \tau_{m}: G_{m} \rightarrow D_{m}\left(\bar{K}_{\bullet}\right)$ has two components

$$
\tau_{0}^{0}: G_{0} \longrightarrow \operatorname{Aut}\left(K_{0} / K_{1}\right), \quad \tau_{0}^{1}: G_{0} \longrightarrow \operatorname{Aut}\left(K_{1}^{\mathrm{ab}}\right)
$$

for $m=0$, and

$$
\tau_{m}^{0}: G_{m} \longrightarrow Z^{1}\left(K_{0} / K_{1}, \operatorname{Lie}_{m}\left(K_{1}^{\mathrm{ab}}\right)\right), \quad \tau_{m}^{1}: G_{m} \longrightarrow \operatorname{Hom}\left(K_{1}^{\mathrm{ab}}, \operatorname{Lie}_{m+1}\left(K_{1}^{\mathrm{ab}}\right)\right)
$$

for $m \geq 1$. Furthermore, these two components are related to each other by

$$
\tau_{m}^{1}(g)\left({ }^{a} b\right)= \begin{cases}\tau_{0}^{0}(g)(a)\left(\tau_{0}^{1}(g)(b)\right) & (m=0), \\ {\left[\tau_{m}^{0}(g)(a),{ }^{a} b\right]+{ }^{a}\left(\tau_{m}^{1}(g)(b)\right)} & (m \geq 1)\end{cases}
$$

for $g \in G_{m}, a \in K, b \in K_{1}$. Note also that

$$
\operatorname{ker} \tau_{m}^{0}=G_{m+1}^{0}, \quad \operatorname{ker} \tau_{m}^{1}=G_{m+1}^{1}=G_{m+1} \quad(m \geq 0) .
$$

Proposition 10.5. The homomorphism $\tau_{0}^{1}$ restricts to

$$
\left.\tau_{0}^{1}\right|_{G_{1}^{0}}: G_{1}^{0} \longrightarrow \operatorname{Aut}_{\mathbb{Z}\left[K / K_{1}\right]}\left(K_{1}^{\mathrm{ab}}\right) .
$$

For $m \geq 1$, the homomorphism $\tau_{m}^{1}$ restricts to

$$
\left.\tau_{m}^{1}\right|_{G_{m+1}^{0}}: G_{m+1}^{0} \longrightarrow \operatorname{Hom}_{\mathbb{Z}\left[K / K_{1}\right]}\left(K_{1}^{\mathrm{ab}}, \operatorname{Lie}_{m+1}\left(K_{1}^{\mathrm{ab}}\right)\right) .
$$

Proof. This immediately follows from (10.5).

Proposition 10.6. Let $m \geq 1$. There is a map

$$
\tilde{\tau}_{m}^{0}: G_{m}^{0} \longrightarrow Z^{1}\left(K_{0}, \operatorname{Lie}_{m}\left(K_{1}^{\mathrm{ab}}\right)\right)
$$

which is a homomorphism for $m \geq 2$ (resp., a 1-cocycle for $m=1$ ) with kernel $G_{m+1}^{0}$, and which makes the following diagram commute:

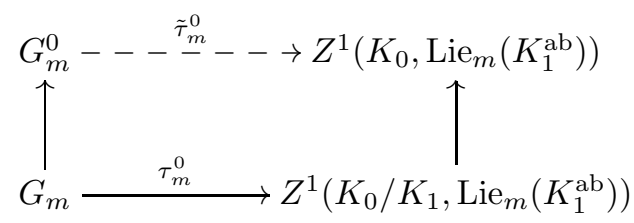


(Here the arrow on the left is the inclusion, and that on the right is induced by the projection $K_{0} \rightarrow K_{0} / K_{1}$.)

Proof. For $g \in G_{m}^{0}$, the map $g^{\prime}: K_{0} \rightarrow K_{m} / K_{m+1} \simeq \operatorname{Lie}_{m}\left(K_{1}^{\mathrm{ab}}\right)$ defined by $g^{\prime}(x)=[g, x]$ is a 1-cocycle. Thus the map $\tilde{\tau}_{m}^{0}: G_{m}^{0} \rightarrow Z^{1}\left(K_{0}, \operatorname{Lie}_{m}\left(K_{1}^{\text {ab }}\right)\right)$ defined by $\tilde{\tau}_{m}^{0}(g)=g^{\prime}$ makes the diagram (10.6) commute. For $g, h \in G_{m}^{0}$ and $x \in K_{0}$, we have

$$
(g h)^{\prime}(x)=[g h, x] K_{m+1}={ }^{g}[h, x][g, x] K_{m+1}={ }^{g}\left(h^{\prime}(x)\right)+g^{\prime}(x) .
$$

Hence, $\tilde{\tau}_{m}^{0}$ is a 1-cocycle for $m=1$, and a homomorphism for $m>1$. Clearly, its kernel is $G_{m+1}^{0}$.

We now illustrate the above constructions with a few examples.

Example 10.7. As in Example 9.5, we consider the mapping class group $G=$ $\operatorname{MCG}\left(\Sigma_{g, 1}, \partial \Sigma_{g, 1}\right)$ acting on $K=\pi_{1}\left(\Sigma_{g, 1}, \star\right)$. If $H:=K_{1}$ is a characteristic subgroup of $K$, then the filtration $\left(G_{m}^{0}\right)_{m \geq 1}$ of $G_{1}^{0}$ coincides with the "higher order Johnson filtration" defined by McNeill [20], and her "higher order Johnson homomorphism" $\tau_{m}^{H}$ coincides with our $\left.\tau_{m-1}^{1}\right|_{G_{m}^{0}}$ for $m \geq 2$. When $H=\Gamma_{2} K$, the subgroup $G_{1}^{0}$ of $G$ is the Torelli group, and $G_{2}^{0}$ is the kernel of the so-called "Magnus representation": the study of this case is carried out in [20].

Example 10.8. Let $K_{0}=\left\langle x_{1}, \ldots, x_{p}, y_{1}, \ldots, y_{q}\right\rangle$ be the free group of rank $p+q$, $p, q \geq 0$. Set $K_{1}=\left\langle\left\langle x_{1}, \ldots, x_{p}\right\rangle\right\rangle \triangleleft K_{0}$. We have $K_{0} / K_{1} \simeq F_{q}:=\left\langle y_{1}, \ldots, y_{q}\right\rangle$. Let $K_{*}$ be the extended $\mathrm{N}$-series defined by (10.1). We call

$$
G=\operatorname{Aut}\left(K_{*}\right)=\left\{f \in \operatorname{Aut}\left(K_{0}\right) \mid f\left(K_{1}\right)=K_{1}\right\}
$$

the fake handlebody group of type $(p, q)$, and

$$
G_{1}^{0}=\operatorname{ker}\left(G \longrightarrow \operatorname{Aut}\left(F_{q}\right)\right)
$$

the fake twist group of type $(p, q)$; see Example 10.9 below to clarify this terminology. If $p \geq 1$ and $(p, q) \neq(1,0)$, then $K_{1}$ is a non-abelian free group, and Theorem 10.2 applies. We will study these groups in more details in [10] using the Johnson homomorphisms $\left(\tau_{m}^{1}\right)_{m \geq 0}$ and $\left(\tilde{\tau}_{m}^{0}\right)_{m \geq 0}$ defined on the two nested filtrations $\left(G_{m}\right)_{m \geq 0}$ and $\left(G_{m}^{0}\right)_{m \geq 0}$, respectively.

Example 10.9. Let $V_{g}$ be a handlebody of genus $g \geq 1$, fix a disk $S \subset \partial V_{g}$ and let $\Sigma_{g, 1}=\partial V_{g} \backslash \operatorname{int}(S)$. Let $\star \in \partial \Sigma_{g, 1}$ and set

$$
K_{0}=\pi_{1}\left(\Sigma_{g, 1}, \star\right) \quad \text { and } K_{1}=\operatorname{ker}\left(i_{*}: \pi_{1}\left(\Sigma_{g, 1}, \star\right) \longrightarrow \pi_{1}\left(V_{g}, \star\right)\right) \text {, }
$$

where $i_{*}$ is induced by the inclusion $i: \Sigma_{g, 1} \hookrightarrow V_{g}$. Let $\operatorname{MCG}\left(\Sigma_{g, 1}, \partial \Sigma_{g, 1}\right)$ act on $K_{0}$ in the canonical way. The subgroup

$$
G=\left\{f \in \operatorname{MCG}\left(\Sigma_{g, 1}, \partial \Sigma_{g, 1}\right) \mid f_{*}\left(K_{1}\right)=K_{1}\right\}
$$

is usually called the handlebody group, since it is the image of $\operatorname{MCG}\left(V_{g}, S\right)$ in $\operatorname{MCG}\left(\Sigma_{g, 1}, \partial \Sigma_{g, 1}\right)$ by the restriction homomorphism (which is injective). The subgroup

$$
\begin{aligned}
G_{1}^{0} & =\operatorname{ker}\left(G \longrightarrow \operatorname{Aut}\left(K_{0} / K_{1}\right)\right) \\
& \simeq \operatorname{ker}\left(\operatorname{MCG}\left(V_{g}, S\right) \longrightarrow \operatorname{Aut}\left(\pi_{1}\left(V_{g}, \star\right)\right)\right),
\end{aligned}
$$

usually called the twist group, is generated by Dehn twists along the boundaries of 2-disks properly embedded in $V_{g} \backslash S$ [19]. The present example corresponds to Example 10.8 with $p=q=g$, where the basis $\left(x_{1}, \ldots, x_{g}, y_{1}, \ldots, y_{g}\right)$ of $K_{0}$ 
is a system of meridians and parallels on $\Sigma_{g, 1}$, and the automorphisms of $K_{0}$ are required to fix the homotopy class of $\partial \Sigma_{g, 1}$. We will prove in [10] that this boundary condition implies that the two nested filtrations (10.4) on $G$ agree:

$$
G_{m}=G_{m}^{0} \quad \text { for all } m \geq 0 .
$$

In this case, the Johnson homomorphisms $\left(\tau_{m}^{1}\right)_{m \geq 0}$ and $\left(\tilde{\tau}_{m}^{0}\right)_{m \geq 0}=\left(\tau_{m}^{0}\right)_{m \geq 0}$ are interchangeable and correspond to the "tree reduction" of the Kontsevich-type functor $Z$ introduced in [9]. Moreover, the maps $\left.\tau_{m}^{1}\right|_{G_{m+1}^{0}}$ given in Proposition 10.5 are trivial.

10.2. $\mathrm{N}$-series determined by $K_{1}$ and $K_{2}$. Let $K=K_{1}$ be a group, and let $K_{2} \triangleleft K$ with $K_{2} \geq[K, K]$. Let $K_{+}=\left(K_{m}\right)_{m \geq 1}$ be the smallest N-series of $K$ with these $K_{1}$ and $K_{2}$, i.e., $K_{+}$is defined by

$$
K_{m}=\left[K_{m-1}, K_{1}\right] \cdot\left[K_{m-2}, K_{2}\right]
$$

inductively for $m \geq 3$. Note that

$$
\Gamma_{m} K \subset K_{m} \subset \Gamma_{\lceil m / 2\rceil} K
$$

for $m \geq 1$, where $\lceil m / 2\rceil=\min \{n \in \mathbb{Z} \mid n \geq m / 2\}$. Extend $K_{+}$to an extended N-series $K_{*}=\left(K_{m}\right)_{m \geq 0}$ with $K_{0}=K_{1}$.

Let a group $G$ act on $K$ in such a way that ${ }^{G} K_{2}=K_{2}$. Then each $g \in G$ satisfies $g\left(K_{j}\right) \subset K_{j}$ for all $j \geq 3$, as can be verified inductively using (10.7). Hence $G$ acts on $K_{*}$ and we can consider the induced Johnson filtration $\mathcal{F}_{*}^{K_{*}}(G)$. It has the following description. Set

$$
G_{m}=\left\{g \in G \mid\left[g, K_{1}\right] \subset K_{m+1},\left[g, K_{2}\right] \subset K_{m+2}\right\} \quad \text { for } m \geq 0 .
$$

Proposition 10.10. We have $\mathcal{F}_{m}^{K_{*}}(G)=G_{m}$ for all $m \geq 0$. Hence $G_{*}=\left(G_{m}\right)_{m \geq 0}$ is an extended $N$-series.

Proof. Obviously, $\mathcal{F}_{m}^{K_{*}}(G) \subset G_{m}$ and $G_{0}=G=\mathcal{F}_{0}^{K_{*}}(G)$.

It remains to prove $G_{m} \subset \mathcal{F}_{m}^{K_{*}}(G)$ for $m \geq 1$. It suffices to check that if $g \in G$ satisfies $\left[g, K_{1}\right] \subset K_{m+1}$ and $\left[g, K_{2}\right] \subset K_{m+2}$, then we have $\left[g, K_{i}\right] \subset K_{m+i}$ for all $i \geq 1$. This is obvious for $i=1,2$. The case $i \geq 3$ is proved by an induction using (10.7), similarly to the proof of Proposition 9.1 in the case $K_{+}=\Gamma_{+} K$.

By Corollary 8.1, we have an injective morphism of eg-Lie algebras

$$
\bar{\tau}_{\bullet}: \bar{G}_{\bullet} \longrightarrow \operatorname{Der} \bullet\left(\bar{K}_{+}\right) \text {. }
$$

In contrast with Section 10.1, the graded Lie algebra $\bar{K}_{+}$is not generated by its degree 1 part. Thus, Proposition 7.1 does not apply and $t_{\bullet}: \operatorname{Der}_{\bullet}\left(\bar{K}_{+}\right) \rightarrow D_{\bullet}\left(\bar{K}_{+}\right)$ might not be injective. Nonetheless, $\bar{K}_{+}$is generated by its degree 1 and 2 parts. This observation motivates the following definitions.

Let $L_{+}$be a graded Lie algebra, and let $A$ be a subgroup of $L_{2}$ such that $L_{2}=\left[L_{1}, L_{1}\right]+A$. We define a graded group $D_{\bullet}\left(L_{+}, A\right)$ as follows. For $m \geq 1$, consider the abelian group

$$
D_{m}\left(L_{+}, A\right)=\operatorname{Hom}\left(L_{1}, L_{m+1}\right) \times \operatorname{Hom}\left(A, L_{m+2}\right)
$$

and, for $m=0$, set

$$
D_{0}\left(L_{+}, A\right)=\left\{(u, v) \in \operatorname{Aut}\left(L_{1}\right) \times \operatorname{Hom}\left(A, L_{2}\right)\right.
$$




$$
\left.\mid \begin{array}{l}
\text { the map }\left[x_{1}, y_{1}\right]+a \longmapsto\left[u\left(x_{1}\right), u\left(y_{1}\right)\right]+v(a) \\
\text { defines an automorphism of }\left[L_{1}, L_{1}\right]+A=L_{2}
\end{array}\right\} .
$$

The subgroup

$$
\left\{\left(d_{1}, d_{2}\right) \in \operatorname{Aut}\left(L_{1}\right) \times \operatorname{Aut}\left(L_{2}\right) \mid d_{2}([b, c])=\left[d_{1}(b), d_{1}(c)\right] \text { for } b, c \in L_{1}\right\}
$$

of $\operatorname{Aut}\left(L_{1}\right) \times \operatorname{Aut}\left(L_{2}\right)$ is mapped bijectively onto $D_{0}\left(L_{+}, A\right)$ by $\left(d_{1}, d_{2}\right) \mapsto\left(d_{1},\left.d_{2}\right|_{A}\right)$. Hence $D_{0}\left(L_{+}, A\right)$ inherits from $\operatorname{Aut}\left(L_{1}\right) \times \operatorname{Aut}\left(L_{2}\right)$ a group structure. For every $m \geq 0$, there is a homomorphism

$$
t_{m}: \operatorname{Der}_{m}\left(L_{+}\right) \longrightarrow D_{m}\left(L_{+}, A\right),\left(d_{i}\right)_{i \geq 1} \longmapsto\left(d_{1},\left.d_{2}\right|_{A}\right) .
$$

Clearly, $t_{\bullet}=\left(t_{m}\right)_{m \geq 0}$ is injective if the graded Lie algebra $L_{+}$is generated by its degree 1 and 2 parts (and, so, by $L_{1} \oplus A$ ). Furthermore, $t_{\bullet}$ is bijective if $L_{+}$is freely generated by $L_{1} \oplus A$, where $L_{1}$ and $A$ are in degree 1 and 2, respectively. Hence, in this case, there is a unique eg-Lie algebra structure on $D_{\bullet}\left(L_{+}, A\right)$ such that $t_{\bullet}$ is an eg-Lie algebra isomorphism.

Now, let $\bar{K}_{+}$be freely generated by $B=\bar{K}_{1}$ and a subgroup $A$ of $\bar{K}_{2}$. Then, the previous paragraph gives an injective eg-Lie algebra morphism

$$
\bar{G}_{\bullet} \stackrel{\bar{\tau}_{\bullet}}{\longrightarrow} \operatorname{Der} \bullet\left(\bar{K}_{+}\right) \stackrel{t_{\bullet}}{\simeq} D \bullet\left(\bar{K}_{+}, A\right) .
$$

The $m$ th Johnson homomorphism $t_{m} \tau_{m}: G_{m} \rightarrow D_{m}\left(\bar{K}_{+}, A\right)$ has two components

$$
\tau_{0}^{1}: G_{0} \longrightarrow \operatorname{Aut}(B), \quad \tau_{0}^{2}: G_{0} \longrightarrow \operatorname{Hom}\left(A, \Lambda^{2} B\right) \times \operatorname{Aut}(A)
$$

for $m=0$, and

$$
\tau_{m}^{1}: G_{m} \longrightarrow \operatorname{Hom}\left(B, \operatorname{Lie}_{m+1}(B ; A)\right), \quad \tau_{m}^{2}: G_{m} \longrightarrow \operatorname{Hom}\left(A, \operatorname{Lie}_{m+2}(B ; A)\right)
$$

for $m \geq 1$. Here $\operatorname{Lie}(B ; A)$ denotes the graded Lie algebra freely generated by $B \oplus A$, where $B$ and $A$ are in degree 1 and 2 , respectively.

We illustrate the above constructions with a few examples. The following lemma is easily deduced from [15, Proposition 1].

Lemma 10.11. Let $K=K_{1}=\left\langle x_{1}, \ldots, x_{p}, y_{1}, \ldots, y_{q}\right\rangle$ be a free group of rank $p+q$ with $p, q \geq 0$, and let

$$
K_{2}=\Gamma_{2} K \cdot\left\langle\left\langle x_{1}, \ldots, x_{p}\right\rangle\right\rangle=\operatorname{ker}\left(K \rightarrow\left\langle y_{1}, \ldots, y_{q}\right\rangle^{\mathrm{ab}}\right) .
$$

Then the graded Lie algebra $\bar{K}_{+}$is freely generated by $y_{1} K_{2}, \ldots, y_{q} K_{2}$ in degree 1 and by $x_{1} K_{3}, \ldots, x_{p} K_{3}$ in degree 2 .

Example 10.12. This generalizes Example 9.5. Let $\Sigma_{g, 1}^{p}$ be the surface $\Sigma_{g, 1}$ with $p \geq 0$ punctures, and let $i: \Sigma_{g, 1}^{p} \rightarrow \Sigma_{g, 1}$ be the inclusion. Set $K=K_{1}=\pi_{1}\left(\Sigma_{g, 1}^{p}, \star\right)$, where $\star \in \partial \Sigma_{g, 1}^{p}=\partial \Sigma_{g, 1}$, and

$$
K_{2}=\operatorname{ker}\left(\pi_{1}\left(\Sigma_{g, 1}^{p}, \star\right) \stackrel{i_{*}}{\longrightarrow} \pi_{1}\left(\Sigma_{g, 1}, \star\right) \longrightarrow \pi_{1}\left(\Sigma_{g, 1}, \star\right)^{\mathrm{ab}} \simeq H_{1}\left(\Sigma_{g, 1} ; \mathbb{Z}\right)\right) .
$$

The smallest N-series $K_{+}=\left(K_{m}\right)_{m \geq 1}$ with these $K_{1}$ and $K_{2}$ is known as the weight filtration. It was introduced by Kaneko [14] in the framework of pro- $\ell$ groups following ideas of Oda, and has been studied by several authors including Nakamura and Tsunogai [26], and Asada and Nakamura [3].

Set $B=K_{1} / K_{2}=H_{1}\left(\Sigma_{g, 1} ; \mathbb{Z}\right)$ and

$$
A=\operatorname{ker}\left(i_{*}: H_{1}\left(\Sigma_{g, 1}^{p} ; \mathbb{Z}\right) \longrightarrow H_{1}\left(\Sigma_{g, 1} ; \mathbb{Z}\right)\right) .
$$


We regard $A$ as a subgroup of $K_{2} / K_{3}$ as follows. Let $x_{1}, \ldots, x_{p} \in K$ be represented by loops (based at $\star$ ) around the $p$ punctures. Since $A$ is free abelian with basis $\left[x_{1}\right], \ldots,\left[x_{p}\right]$, there is a unique homomorphism $j: A \rightarrow K_{2} / K_{3}$ defined by $j\left(\left[x_{i}\right]\right)=$ $x_{i} K_{3}$; one easily checks that $j$ does not depend on the choice of $x_{1}, \ldots, x_{p}$. By Lemma $10.11, j$ is injective and the graded Lie algebra $\bar{K}_{+}$is freely generated by $B \oplus j(A)$, where $B$ and $j(A)$ are in degree 1 and 2 , respectively.

The mapping class group $G=\operatorname{MCG}\left(\Sigma_{g, 1}^{p}, \partial \Sigma_{g, 1}^{p}\right)$ acts on $K$ in the canonical way, and we have ${ }^{G} K_{2}=K_{2}$. The extended N-series $G=G_{0} \geq G_{1} \geq G_{2} \geq \cdots$ coincides with the filtration

$$
\Gamma_{g,[p+1]}^{*} \geq \Gamma_{g, p+1}^{*}(1) \geq \Gamma_{g, p+1}^{*}(2) \geq \cdots
$$

in [3, $§ 2.1]$. Furthermore, for $m \geq 1$, the Johnson homomorphism $t_{m} \tau_{m}=\left(\tau_{m}^{1}, \tau_{m}^{2}\right)$ is essentially the same as the homomorphism $c_{m}$ in $[3, \S 2.2]$.

There is a short exact sequence

$$
1 \longrightarrow B_{p}\left(\Sigma_{g, 1}\right) \longrightarrow G \longrightarrow \operatorname{MCG}\left(\Sigma_{g, 1}, \partial \Sigma_{g, 1}\right) \longrightarrow 1,
$$

where $B_{p}\left(\Sigma_{g, 1}\right)$ is the braid group in $\Sigma_{g, 1}$ on $p$ strands. Thus, the homomorphisms $\tau_{m}^{i}$ (for $m \geq 1, i=1,2$ ) generalize both the "classical" Johnson homomorphisms $(p=0)$ and Milnor's $\mu$-invariants $(g=0)$. The former are contained in the "tree reduction" of the LMO functor [5], while the latter are contained in the "tree reduction" of the Kontsevich integral [8]. It seems possible to describe diagrammatically the generalized Johnson homomorphisms $\tau_{m}^{i}$ for any $g, p \geq 0$ and to relate them to the "tree reduction" of the extended LMO functor introduced in [27].

Example 10.13. As in Example 10.9, consider a handlebody $V_{g}$ of genus $g \geq 1$ and a surface $\Sigma_{g, 1} \subset \partial V_{g}$ of genus $g$. Set $K=K_{1}=\pi_{1}\left(\Sigma_{g, 1}, \star\right)$ and

$$
K_{2}=\operatorname{ker}\left(\pi_{1}\left(\Sigma_{g, 1}, \star\right) \stackrel{i_{*}}{\longrightarrow} \pi_{1}\left(V_{g}, \star\right) \longrightarrow \pi_{1}\left(V_{g}, \star\right)^{\mathrm{ab}} \simeq H_{1}\left(V_{g} ; \mathbb{Z}\right)\right) .
$$

The smallest $\mathrm{N}$-series $K_{+}=\left(K_{m}\right)_{m \geq 1}$ with these $K_{1}$ and $K_{2}$ is given by

$$
K_{2}=\Gamma_{2} K \cdot \mathbf{A}, \quad K_{3}=\Gamma_{3} K \cdot[K, \mathbf{A}], \quad \text { etc. }
$$

where $\mathbf{A}=\operatorname{ker}\left(i_{*}: \pi_{1}\left(\Sigma_{g, 1}, \star\right) \longrightarrow \pi_{1}\left(V_{g}, \star\right)\right)$. Let

$$
A=\operatorname{ker}\left(i_{*}: H_{1}\left(\Sigma_{g, 1} ; \mathbb{Z}\right) \longrightarrow H_{1}\left(V_{g} ; \mathbb{Z}\right)\right) \text { and } B=H_{1}\left(V_{g} ; \mathbb{Z}\right) .
$$

Identify $B$ with $K_{1} / K_{2}$, and let $j: A \rightarrow K_{2} / K_{3}$ be the canonical homomorphism

$$
A \simeq \frac{\Gamma_{2} K \cdot \mathbf{A}}{\Gamma_{2} K} \simeq \frac{\mathbf{A}}{\Gamma_{2} K \cap \mathbf{A}}=\frac{\mathbf{A}}{[K, \mathbf{A}]} \longrightarrow \frac{K_{2}}{K_{3}} .
$$

Then, by Lemma 10.11, $j$ is injective and the graded Lie algebra $\bar{K}_{+}$is freely generated by $B \oplus j(A)$, where $B$ and $j(A)$ are in degree 1 and 2 , respectively.

The subgroup $G$ of $\operatorname{MCG}\left(\Sigma_{g, 1}, \partial \Sigma_{g, 1}\right)$ that preserves the Lagrangian subgroup $A \subset H_{1}\left(\Sigma_{g, 1} ; \mathbb{Z}\right)$ is usually called the Lagrangian mapping class group of $\Sigma_{g, 1}$. It acts on $K$ in the canonical way and satisfies ${ }^{G} K_{2}=K_{2}$. Hence we obtain an extended N-series $G_{*}=\left(G_{m}\right)_{m \geq 0}$, which is the Johnson filtration induced by $K_{*}$. The generalized Johnson homomorphisms $\tau_{m}^{i}$ (for $m \geq 0, i=1,2$ ) will be studied by Vera [40] in relation with the "tree reduction" of the LMO functor introduced in [5]. This is also connected to the "Lagrangian" versions of the Johnson homomorphisms introduced by Levine in $[17,18]$. 


\section{FiLTRATIONS ON GROUP RINGS AND THEIR ASSOCIATED GRADED}

In this section, we consider filtrations on group rings induced by extended Nseries and we compute their associated graded. By a ring we mean an associative ring with unit.

11.1. Filtrations on group rings. A filtered ring $J_{*}=\left(J_{m}\right)_{m \geq 0}$ is a ring $J_{0}$ together with a decreasing sequence

$$
J_{0} \supset J_{1} \supset \cdots \supset J_{k} \supset J_{k+1} \supset \cdots
$$

of additive subgroups such that

$$
J_{m} J_{n} \subset J_{m+n} \text { for } m, n \geq 0 .
$$

Note that $J_{m}$ is an ideal of $J_{0}$ for each $m \geq 0$. The associated graded of $J_{*}$,

$$
\operatorname{gr}_{\bullet}\left(J_{*}\right)=\bigoplus_{k \geq 0} \frac{J_{k}}{J_{k+1}},
$$

has the obvious graded ring structure.

Let $K_{*}$ be an extended N-series, and $\mathbb{Z}\left[K_{0}\right]$ the group ring of $K_{0}$. For $m \geq 1$, we set

$$
I_{m}\left(K_{*}\right)=\operatorname{ker}\left(\mathbb{Z}\left[K_{0}\right] \stackrel{\mathbb{Z}\left[\pi_{m}\right]}{\longrightarrow} \mathbb{Z}\left[K_{0} / K_{m}\right]\right),
$$

where $\pi_{m}: K_{0} \rightarrow K_{0} / K_{m}$ is the projection. We associate to $K_{*}$ the filtered ring

$$
J_{*}\left(K_{*}\right)=\left(J_{m}\left(K_{*}\right)\right)_{m \geq 0}
$$

defined by $J_{0}\left(K_{*}\right)=\mathbb{Z}\left[K_{0}\right]$ and by

$$
J_{m}\left(K_{*}\right)=\sum_{\substack{m_{1}, \ldots, m_{p} \geq 1, p \geq 1 \\ m_{1}+\cdots+m_{p} \geq m}} I_{m_{1}}\left(K_{*}\right) \cdots I_{m_{p}}\left(K_{*}\right) \quad \text { for } m \geq 1 .
$$

Note that $J_{m}\left(K_{*}\right)$ is the ideal of $\mathbb{Z}\left[K_{0}\right]$ generated by the elements $\left(x_{1}-1\right) \cdots\left(x_{p}-1\right)$ for all $x_{1} \in K_{m_{1}}, \ldots, x_{p} \in K_{m_{p}}, m_{1}+\cdots+m_{p} \geq m, m_{1}, \ldots, m_{p} \geq 1, p \geq 1$. For instance, if $K_{*}$ is the extended N-series defined by the lower central series of the group $K_{0}$, then we have $J_{m}\left(K_{*}\right)=I^{m}$, where $I$ is the augmentation ideal of $\mathbb{Z}\left[K_{0}\right]$.

Now we equip the group ring $\mathbb{Z}\left[K_{0}\right]$ with the usual Hopf algebra structure with comultiplication $\Delta$, counit $\epsilon$ and antipode $S$. Since

$$
\Delta\left(I_{k}\left(K_{*}\right)\right) \subset I_{k}\left(K_{*}\right) \otimes \mathbb{Z}\left[K_{0}\right]+\mathbb{Z}\left[K_{0}\right] \otimes I_{k}\left(K_{*}\right)
$$

for $k \geq 0$, we have

$$
\Delta\left(J_{m}\left(K_{*}\right)\right) \subset \sum_{i+j=m} J_{i}\left(K_{*}\right) \otimes J_{j}\left(K_{*}\right) .
$$

Clearly, we have $\epsilon\left(J_{m}\left(K_{*}\right)\right)=0$ and $S\left(J_{m}\left(K_{*}\right)\right)=J_{m}\left(K_{*}\right)$ for all $m \geq 1$. Hence $J_{*}\left(K_{*}\right)$ has the structure of a filtered Hopf algebra and, consequently, the associated graded

$$
\operatorname{gr}_{\bullet}\left(J_{*}\left(K_{*}\right)\right)=\bigoplus_{i \geq 0} \frac{J_{i}\left(K_{*}\right)}{J_{i+1}\left(K_{*}\right)}
$$

has the structure of a graded Hopf algebra. 
11.2. Universal enveloping algebras of eg-Lie algebras. Let $L_{\bullet}$ be an eg-Lie algebra. Then we have two Hopf algebras $\mathbb{Z}\left[L_{0}\right]$ and $U\left(L_{+}\right)$, the universal enveloping algebra of $L_{+}$. The action of $L_{0}$ on $L_{+}$induces an action of $\mathbb{Z}\left[L_{0}\right]$ on $U\left(L_{+}\right)$. The universal enveloping algebra $U\left(L_{\bullet}\right)$ of $L_{\bullet}$ is defined to be the crossed product (or the smash product) $U\left(L_{+}\right) \sharp \mathbb{Z}\left[L_{0}\right]$ of $U\left(L_{+}\right)$and $\mathbb{Z}\left[L_{0}\right]$, which is the Hopf algebra structure on $U\left(L_{+}\right) \otimes \mathbb{Z}\left[L_{0}\right]$ with multiplication and comultiplication defined by

$$
\begin{gathered}
(u \otimes g) \cdot\left(u^{\prime} \otimes g\right)=u\left({ }^{g} u^{\prime}\right) \otimes g g^{\prime} \quad \text { for } u, u^{\prime} \in U\left(L_{+}\right), g, g^{\prime} \in L_{0}, \\
\Delta(u \otimes g)=\sum\left(u^{\prime} \otimes g\right) \otimes\left(u^{\prime \prime} \otimes g\right) \quad \text { for } u \in U\left(L_{+}\right), g \in L_{0},
\end{gathered}
$$

where $\Delta(u)=\sum u^{\prime} \otimes u^{\prime \prime}$.

We usually write $u \otimes g=u \cdot g$ in $U\left(L_{\bullet}\right)$, and we regard both $U\left(L_{+}\right)$and $\mathbb{Z}\left[L_{0}\right]$ as Hopf subalgebras of $U\left(L_{\bullet}\right)$. By (11.3) we have

$$
g \cdot u \cdot g^{-1}={ }^{g} u \quad \text { for } g \in L_{0}, u \in U\left(L_{+}\right) .
$$

The grading of $L_{+}$makes $U\left(L_{\bullet}\right)$ a graded Hopf algebra.

11.3. Taking rational coefficients. Here we carry out some of the previous constructions over $\mathbb{Q}$. First of all, there is a notion of filtered $\mathbb{Q}$-algebra similar to that of filtered ring in Section 11.1. For each extended N-series $K_{*}$, there is a filtration $J_{*}^{\mathbb{Q}}\left(K_{*}\right)$ of $\mathbb{Q}\left[K_{0}\right]$ whose definition is parallel to that of $J_{*}\left(K_{*}\right)$.

We define an eg-Lie $\mathbb{Q}$-algebra L in the same way as an eg-Lie algebra in Section 4.2: here $L_{+}$is assumed to be a graded Lie algebra over $\mathbb{Q}$. For each extended N-series $K_{*}$, there is an associated eg-Lie $\mathbb{Q}$-algebra $\operatorname{gr}_{\bullet} \mathbb{Q}\left(K_{*}\right)$ defined by $\operatorname{gr}_{0}^{\mathbb{Q}}\left(K_{*}\right)=K_{0} / K_{1}$ and $\operatorname{gr}_{m}^{\mathbb{Q}}\left(K_{*}\right)=\left(K_{m} / K_{m+1}\right) \otimes \mathbb{Q}$ for $m \geq 1$.

The contents of Section 5.2 can also be adapted to an eg-Lie $\mathbb{Q}$-algebra $L_{\bullet}$. Thus we define the derivation eg-Lie $\mathbb{Q}$-algebra $\operatorname{Der} \bullet\left(L_{\bullet}\right)$ of $L_{\bullet}$, and Theorem 5.3 works over $\mathbb{Q}$ as well.

Finally, the definitions of Section 11.2 work also over $\mathbb{Q}$. The universal enveloping algebra $U\left(L_{\bullet}\right)$ of an eg-Lie $\mathbb{Q}$-algebra $L_{\bullet}$ is the $\mathbb{Q}$-vector space $U\left(L_{+}\right) \otimes_{\mathbb{Q}} \mathbb{Q}\left[L_{0}\right]$ with multiplication - defined by (11.3). Note that $U\left(L_{\bullet}\right)$ has a graded Hopf $\mathbb{Q}$ algebra structure. Let $\hat{U}\left(L_{\bullet}\right)$ denote its degree-completion, which is a complete Hopf algebra.

Lemma 11.1. For every eg-Lie $\mathbb{Q}$-algebra $L_{\bullet}$, the group-like part of $\hat{U}\left(L_{\bullet}\right)$ is

$$
\left\{\exp (\ell) \cdot g \mid \ell \in \hat{L}_{+}, g \in L_{0}\right\},
$$

where $\hat{L}_{+}$denotes the degree-completion of $L_{+}$.

Proof. It is easy to see that $\exp (\ell) \cdot g$ is group-like in $\hat{U}\left(L_{\bullet}\right)$ for $\ell \in \hat{L}_{+}, g \in L_{0}$.

Conversely, let $x$ be a group-like element of $\hat{U}\left(L_{\bullet}\right)$. We can write

$$
x=\sum_{g \in L_{0}} x_{g} \cdot g,
$$

where $x_{g} \in \hat{U}\left(L_{+}\right)$are uniquely determined by $x$, and for each $m \geq 0$ there are only finitely many $g \in L_{0}$ such that the degree $m$ part of $x_{g}$ is non-zero. We have

$$
\Delta(x)=\sum_{g \in L_{0}} \sum\left(x_{g}^{\prime} \cdot g\right) \otimes\left(x_{g}^{\prime \prime} \cdot g\right),
$$


where $\Delta\left(x_{g}\right)=\sum x_{g}^{\prime} \otimes x_{g}^{\prime \prime}$. We also have

$$
x \otimes x=\sum_{g, h \in L_{0}}\left(x_{g} \cdot g\right) \otimes\left(x_{h} \cdot h\right),
$$

Since $\Delta(x)=x \otimes x$, it follows that

$$
\begin{aligned}
\Delta\left(x_{g}\right)=x_{g} \otimes x_{g} & \text { for all } g \in L_{0}, \\
x_{g} \otimes x_{h}=0 & \text { for all } g, h \in L_{0}, g \neq h .
\end{aligned}
$$

Since $x \neq 0$, there is $g \in L_{0}$ such that $x=x_{g} \cdot g$ and $x_{g}$ is group-like. Hence $\ell=\log \left(x_{g}\right)$ is primitive in $\hat{U}\left(L_{+}\right)$. Since the primitive part of $U\left(L_{+}\right)$is $L_{+}$, the element $\ell$ belongs to the degree-completion of $L_{+}$.

11.4. Quillen's description of the associated graded of a group ring. A wellknown result of Quillen describes the associated graded of a group ring filtered by powers of the augmentation ideal [35]. This result is generalized to the filtration of a group ring induced by any extended N-series, as follows.

Theorem 11.2. Let $K_{*}$ be an extended $N$-series. There is a (unique) ring homomorphism

$$
\Upsilon: U\left(\operatorname{gr}_{\bullet}\left(K_{*}\right)\right) \longrightarrow \operatorname{gr} \bullet\left(J_{*}\left(K_{*}\right)\right)
$$

defined by $\Upsilon\left(g K_{1}\right)=g+J_{1}\left(K_{*}\right)$ for $g \in K_{0}$ and by $\Upsilon\left(x K_{i+1}\right)=(x-1)+J_{i+1}\left(K_{*}\right)$ for $x \in K_{i}, i \geq 1$. Furthermore, the rational version of $\Upsilon$

$$
\Upsilon^{\mathbb{Q}}: U\left(\operatorname{gr}_{\bullet}^{\mathbb{Q}}\left(K_{*}\right)\right) \longrightarrow \operatorname{gr}_{\bullet}\left(J_{*}^{\mathbb{Q}}\left(K_{*}\right)\right)
$$

is a $\mathbb{Q}$-algebra isomorphism.

Proof. The N-series $K_{+}=\left(K_{m}\right)_{m \geq 1}$ defined by $K_{*}$ induces a filtration

$$
J_{+}^{\prime}\left(K_{+}\right)=\left(J_{m}^{\prime}\left(K_{+}\right)\right)_{m \geq 1},
$$

where $J_{m}^{\prime}\left(K_{+}\right)$is the subgroup of $\mathbb{Z}\left[K_{1}\right]$ spanned by the elements $\left(x_{1}-1\right) \cdots\left(x_{p}-1\right)$ for all $x_{1} \in K_{m_{1}}, \ldots, x_{p} \in K_{m_{p}}, m_{1}+\cdots+m_{p} \geq m, m_{1}, \ldots, m_{p} \geq 1, p \geq 1$. (It is an ideal of $\mathbb{Z}\left[K_{1}\right]$ contained in $J_{m}\left(K_{*}\right)$.) Let

$$
\operatorname{gr}_{+}\left(J_{+}^{\prime}\left(K_{+}\right)\right)=\bigoplus_{m \geq 1} \frac{J_{m}^{\prime}\left(K_{+}\right)}{J_{m+1}^{\prime}\left(K_{+}\right)}
$$

be the associated graded ring, and let

$$
\operatorname{gr}_{+}\left(K_{+}\right)=\bigoplus_{m \geq 1} \frac{K_{m}}{K_{m+1}}
$$

be the graded Lie algebra associated to the $N$-series $K_{+}$. It is easily checked that the graded abelian group homomorphism

$$
\operatorname{gr}_{+}\left(K_{+}\right) \longrightarrow \operatorname{gr}_{+}\left(J_{+}^{\prime}\left(K_{+}\right)\right),\left(x K_{m+1}\right) \longmapsto(x-1)+J_{m+1}^{\prime}\left(K_{+}\right)
$$

preserves the Lie bracket and hence induces a ring homomorphism

$$
\Upsilon^{\prime}: U\left(\operatorname{gr}_{+}\left(K_{+}\right)\right) \longrightarrow \operatorname{gr}_{+}\left(J_{+}^{\prime}\left(K_{+}\right)\right)
$$

By composing it with the canonical map $\operatorname{gr}_{+}\left(J_{+}^{\prime}\left(K_{+}\right)\right) \rightarrow \operatorname{gr}_{+}\left(J_{*}\left(K_{*}\right)\right)$, we obtain a ring homomorphism

$$
\Upsilon: U\left(\operatorname{gr}_{+}\left(K_{*}\right)\right)=U\left(\operatorname{gr}_{+}\left(K_{+}\right)\right) \longrightarrow \operatorname{gr} \bullet\left(J_{*}\left(K_{*}\right)\right) .
$$


Besides, the inverse of the canonical isomorphism $\mathbb{Z}\left[K_{0}\right] / J_{1}\left(K_{*}\right) \rightarrow \mathbb{Z}\left[\bar{K}_{0}\right]$, where $\bar{K}_{0}=K_{0} / K_{1}$, defines a ring homomorphism

$$
\Upsilon: \mathbb{Z}\left[\bar{K}_{0}\right] \longrightarrow \operatorname{gr} \bullet\left(J_{*}\left(K_{*}\right)\right) .
$$

A straightforward computation shows that (11.8) and (11.9) define together a ring homomorphism (11.6) on $U\left(\operatorname{gr}_{\bullet}\left(K_{*}\right)\right)=U\left(\operatorname{gr}_{+}\left(K_{*}\right)\right) \sharp \mathbb{Z}\left[\bar{K}_{0}\right]$.

As a generalization of Quillen's result mentioned above, it is known that the rational version $\Upsilon^{\mathbb{Q}}$ of $\Upsilon^{\prime}$ is an isomorphism [21, Corollary 5.4]. Thus, to conclude that $\Upsilon^{\mathbb{Q}}$ is an isomorphism, it suffices to prove that $\operatorname{gr}_{+}\left(J_{*}\left(K_{*}\right)\right)$ is isomorphic to $\operatorname{gr}_{+}\left(J_{+}^{\prime}\left(K_{+}\right)\right) \otimes \mathbb{Z}\left[\bar{K}_{0}\right]$. Specifically, we need to prove that the group homomorphism

$$
r: \frac{J_{m}^{\prime}\left(K_{+}\right)}{J_{m+1}^{\prime}\left(K_{+}\right)} \otimes \mathbb{Z}\left[\bar{K}_{0}\right] \longrightarrow \frac{J_{m}\left(K_{*}\right)}{J_{m+1}\left(K_{*}\right)}
$$

defined by $r\left(\left(u+J_{m+1}^{\prime}\left(K_{+}\right)\right) \otimes\left(g K_{1}\right)\right)=\left(u g+J_{m+1}\left(K_{*}\right)\right)$ is an isomorphism for each $m \geq 1$. Clearly, $r$ is surjective. To construct a left inverse to $r$, let $\pi: K_{0} \rightarrow \bar{K}_{0}$ denote the canonical projection, and let $s: \bar{K}_{0} \rightarrow K_{0}$ be a set-theoretic section of $\pi$. Then there is a unique group homomorphism

$$
q: \mathbb{Z}\left[K_{0}\right] \longrightarrow \mathbb{Z}\left[K_{1}\right] \otimes \mathbb{Z}\left[\bar{K}_{0}\right]
$$

defined by $q(g)=\left(g(s \pi(g))^{-1}\right) \otimes \pi(g)$ for $g \in K_{0}$. For any $x_{1} \in K_{m_{1}}, \ldots, x_{p} \in K_{m_{p}}$ with $m_{1}+\cdots+m_{p} \geq m, m_{1}, \ldots, m_{p} \geq 1, p \geq 1$, and for any $y \in K_{0}$, we have

$$
q\left(\left(x_{1}-1\right) \cdots\left(x_{p}-1\right) y\right)=\left(x_{1}-1\right) \cdots\left(x_{p}-1\right)\left(y(s \pi(y))^{-1}\right) \otimes \pi(y),
$$

which shows that $q\left(J_{m}\left(K_{*}\right)\right) \subset J_{m}^{\prime}\left(K_{+}\right) \otimes \mathbb{Z}\left[\bar{K}_{0}\right]$. Therefore, $q$ induces a group homomorphism

$$
q: \frac{J_{m}\left(K_{*}\right)}{J_{m+1}\left(K_{*}\right)} \longrightarrow \frac{J_{m}^{\prime}\left(K_{+}\right) \otimes \mathbb{Z}\left[\bar{K}_{0}\right]}{J_{m+1}^{\prime}\left(K_{+}\right) \otimes \mathbb{Z}\left[\bar{K}_{0}\right]} \simeq \frac{J_{m}^{\prime}\left(K_{+}\right)}{J_{m+1}^{\prime}\left(K_{+}\right)} \otimes \mathbb{Z}\left[\bar{K}_{0}\right]
$$

which satisfies $q r=$ id.

Remark 11.3. It is easily verified that $\Upsilon$ preserves the graded Hopf algebra structures. Hence $\Upsilon_{\mathbb{Q}}$ is a graded Hopf $\mathbb{Q}$-algebra isomorphism.

\section{Formality of extended N-Series}

Assuming that an extended N-series $K_{*}$ is "formal" in some sense, we here show that an action of an extended N-series $G_{*}$ on $K_{*}$ has an "infinitesimal" counterpart containing all the Johnson homomorphisms. In this section, we work over $\mathbb{Q}$.

12.1. Formality and expansions. Let $K_{*}$ be an extended N-series and consider the completion

$$
\widehat{\mathbb{Q}\left[K_{*}\right]}=\underbrace{}_{k} \mathbb{Q}\left[K_{0}\right] / J_{k}^{\mathbb{Q}}\left(K_{*}\right)
$$

of the group $\mathbb{Q}$-algebra $\mathbb{Q}\left[K_{0}\right]$ with respect to the rational version $J_{*}^{\mathbb{Q}}\left(K_{*}\right)$ of the filtration (11.2). The filtered Hopf $\mathbb{Q}$-algebra structure of $\mathbb{Q}\left[K_{0}\right]$ extends to a complete Hopf algebra structure on $\widehat{\mathbb{Q}\left[K_{*}\right]}$, whose filtration is denoted by $\hat{J}_{*}^{\mathbb{Q}}\left(K_{*}\right)$. 
An extended N-series $K_{*}$ is said to be formal if the complete Hopf algebra $\widehat{\mathbb{Q}\left[K_{*}\right]}$ is isomorphic to the degree-completion of its associated graded, namely

$$
\widehat{\operatorname{gr}} \bullet\left(J_{*}^{\mathbb{Q}}\left(K_{*}\right)\right)=\prod_{k \geq 0} \frac{J_{k}^{\mathbb{Q}}\left(K_{*}\right)}{J_{k+1}^{\mathbb{Q}}\left(K_{*}\right)},
$$

through an isomorphism whose associated graded is the identity.

Recall that $\hat{U}\left(\operatorname{gr}_{\bullet}^{\mathbb{Q}}\left(K_{*}\right)\right)$ denotes the degree-completion of the universal enveloping algebra of the eg-Lie $\mathbb{Q}$-algebra gr $\mathbb{Q}\left(K_{*}\right)$ associated to the extended N-series $K_{*}$. An expansion of an extended N-series $K_{*}$ is a homomorphism

$$
\theta: K_{0} \longrightarrow \hat{U}\left(\operatorname{gr}_{\bullet}^{\mathbb{Q}}\left(K_{*}\right)\right)
$$

which maps any $x \in K_{i}, i \geq 0$ to a group-like element of the form

$$
\theta(x)= \begin{cases}1+\left(x K_{i+1}\right)+(\operatorname{deg}>i) & \text { if } i>0, \\ \left(x K_{1}\right)+(\operatorname{deg}>0) & \text { if } i=0 .\end{cases}
$$

Example 12.1. Assume that $K_{*}$ is associated with the lower central series of a free group $K_{0}=K_{1}$. Let $\operatorname{Lie}\left(H^{\mathbb{Q}}\right)$ denote the free Lie $\mathbb{Q}$-algebra generated by $H^{\mathbb{Q}}=\left(K_{1} / K_{2}\right) \otimes \mathbb{Q}$ in degree 1 . Then the identity of $H^{\mathbb{Q}}$ extends uniquely to an isomorphism $\operatorname{Lie}\left(H^{\mathbb{Q}}\right) \simeq \operatorname{gr}_{+}^{\mathbb{Q}}\left(K_{*}\right)$ of graded Lie $\mathbb{Q}$-algebras, so that we have a canonical isomorphism of graded Hopf $\mathbb{Q}$-algebras

$$
U\left(\operatorname{gr}_{\bullet}^{\mathbb{Q}}\left(K_{*}\right)\right)=U\left(\operatorname{gr}_{+}^{\mathbb{Q}}\left(K_{*}\right)\right) \simeq U\left(\operatorname{Lie}\left(H^{\mathbb{Q}}\right)\right)=T\left(H^{\mathbb{Q}}\right),
$$

where $T\left(H^{\mathbb{Q}}\right)$ is the tensor algebra generated by $H^{\mathbb{Q}}$ in degree 1 . Hence, in this case, an expansion of $K_{*}$ is a homomorphism $\theta: K_{0} \rightarrow \hat{T}\left(H^{\mathbb{Q}}\right)$ such that

$$
\theta(x)=\exp ([x]+(\text { series of Lie elements of degree }>1))
$$

for all $x \in K_{0}$, where $[x]=\left(x K_{2}\right) \otimes 1 \in H^{\mathbb{Q}}$. For instance, for each basis $b=\left(b_{i}\right)_{i}$ of $K_{0}$, there is a unique expansion $\theta_{b}$ of $K_{*}$ such that $\theta_{b}\left(b_{i}\right)=\exp \left(\left[b_{i}\right]\right)$.

The following establishes the relationship between formality and expansions.

Proposition 12.2. An extended $N$-series $K_{*}$ is formal if and only if it has an expansion.

Proof. Consider the diagram

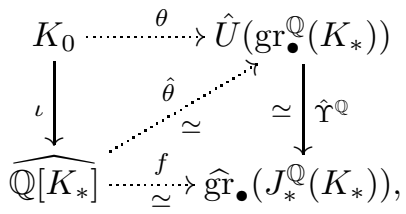

where $\iota$ is the canonical map and $\hat{\Upsilon}^{\mathbb{Q}}$ is the isomorphism in Theorem 11.2.

Assume that $K_{*}$ is formal. Then there is a complete Hopf algebra isomorphism $f$ in (12.3) inducing the identity on the associated graded. The complete Hopf algebra isomorphism $\hat{\theta}:=\left(\hat{\Upsilon}^{\mathbb{Q}}\right)^{-1} f$ satisfies

$$
\hat{\theta}(y)=\left(\Upsilon^{\mathbb{Q}}\right)^{-1}\left(y+J_{m+1}^{\mathbb{Q}}\left(K_{*}\right)\right)+(\operatorname{deg}>m) \quad \text { for } y \in J_{m}^{\mathbb{Q}}\left(K_{*}\right), m \geq 0,
$$

which implies (12.1) for the homomorphism $\theta:=\hat{\theta} \iota$. Since $\iota\left(K_{0}\right)$ is contained in the group-like part of $\widehat{\mathbb{Q}\left[K_{*}\right]}$ and $\hat{\theta}$ preserves the comultiplication, $\theta\left(K_{0}\right)$ is contained in the group-like part of $\hat{U}\left(\operatorname{gr}_{\bullet}^{\mathbb{Q}}\left(K_{*}\right)\right)$. 
Conversely, assume that $K_{*}$ has an expansion, i.e., a homomorphism $\theta$ in (12.3). Extend $\theta$ by linearity to an algebra homomorphism $\theta: \mathbb{Q}\left[K_{0}\right] \rightarrow \hat{U}\left(\operatorname{gr} \mathbb{Q}\left(K_{*}\right)\right)$, which is filtration-preserving by (12.1). Hence it induces a complete algebra homomorphism $\hat{\theta}$ in $(12.3)$. Since $\iota\left(K_{0}\right)$ generates $\widehat{\mathbb{Q}\left[K_{*}\right]}$ as a topological vector space and since $\hat{\theta}$ maps $\iota\left(K_{0}\right)$ into the group-like part of $\hat{U}\left(\operatorname{gr}_{\bullet}^{\mathbb{Q}}\left(K_{*}\right)\right)$, it follows that $\hat{\theta}$ preserves the comultiplication: therefore, $\hat{\theta}$ is a complete Hopf algebra homomorphism. By (12.1), $\hat{\theta}$ induces the isomorphism $\left(\Upsilon^{\mathbb{Q}}\right)^{-1}$ on the associated graded: hence $\hat{\theta}$ is an isomorphism. Thus, $f:=\Upsilon^{\mathbb{Q}} \hat{\theta}$ tells us that $K_{*}$ is formal.

Remark 12.3. Let $\theta$ be an expansion of an extended N-series $K_{*}$. The arguments in the proof of Proposition 12.2 shows that $\theta$ induces a complete Hopf algebra isomorphism

$$
\hat{\theta}: \widehat{\mathbb{Q}\left[K_{+}\right]} \longrightarrow \hat{U}\left(\mathrm{gr}_{+}^{\mathbb{Q}}\left(K_{*}\right)\right)
$$

where $\widehat{\mathbb{Q}\left[K_{+}\right]}$denotes the completion of $\mathbb{Q}\left[K_{1}\right]$ with respect to the rational version of the filtration $J_{+}^{\prime}\left(K_{+}\right)$defined at (11.7).

Remark 12.4. Assume that $K_{*}$ is the extended N-series defined by the lower central series of a group. Then an expansion of $K_{*}$ in our sense is called a "Taylor expansion" in [4] and a "group-like expansion" in [22] (in the case of a free group). Note that $K_{*}$ is formal in our sense if and only if it is "filtered-formal" (over $\mathbb{Q}$ ) in the sense of [39]. Proposition 12.2 is a generalization of [22, Proposition 2.10] and [39, Theorem 8.5].

12.2. Actions of extended $\mathbf{N}$-series in the formal case. Let a group $G$ act on an extended $\mathrm{N}$-series $K_{*}$. This action induces a homomorphism

$$
\rho: G \longrightarrow \operatorname{Aut}\left(\widehat{\mathbb{Q}\left[K_{*}\right]}\right)
$$

with values in the automorphism group of the complete Hopf algebra $\left.\widehat{\mathbb{Q}\left[K_{*}\right.}\right]$. Here, $\rho$ maps each $g \in G$ to the unique automorphism $\rho(g)$ extending the automorphism of $K_{0}$ defined by $x \mapsto{ }^{g} x$.

Now, assume that $K_{*}$ is formal, and fix an expansion $\theta$ of $K_{*}$. According to the proof of Proposition 12.2, $\theta$ extends uniquely to a complete Hopf algebra isomorphism

$$
\hat{\theta}: \widehat{\mathbb{Q}\left[K_{*}\right]} \longrightarrow \hat{U}\left(\bar{K}_{\bullet}^{\mathbb{Q}}\right)
$$

where $U\left(\bar{K}_{\bullet}^{\mathbb{Q}}\right)$ is the universal enveloping algebra of the eg-Lie $\mathbb{Q}$-algebra $\bar{K}_{\bullet}^{\mathbb{Q}}:=$ $\operatorname{gr}_{\bullet}^{\mathbb{Q}}\left(K_{*}\right)$ associated to the extended N-series $K_{*}$. Thus $\theta$ induces a homomorphism

$$
\rho^{\theta}: G \longrightarrow \operatorname{Aut}\left(\hat{U}\left(\bar{K}_{\bullet}^{\mathbb{Q}}\right)\right)
$$

defined by $\rho^{\theta}(g)=\hat{\theta} \rho(g) \hat{\theta}^{-1}$ for $g \in G$.

Furthermore, we assume that $G$ is equipped with an N-series $G_{+}=\left(G_{m}\right)_{m \geq 1}$ and that (the extended N-series corresponding to) $G_{+} \operatorname{acts}$ on $K_{*}$. Recall that $\operatorname{Der}_{+}\left(\bar{K}_{\bullet}^{\mathbb{Q}}\right)$ denotes the derivation graded Lie algebra of the eg-Lie $\mathbb{Q}$-algebra $\bar{K}_{\bullet}^{\mathbb{Q}}$, and let $\widehat{\operatorname{Der}}_{+}\left(\bar{K}_{\bullet}^{\mathbb{Q}}\right)$ denote its degree-completion. Here is the main construction of this section:

Lemma 12.5. Let an $N$-series $G_{+}$of a group $G$ act on a formal extended $N$ series $K_{*}$, and let $\theta$ be an expansion of $K_{*}$. Then, for any $g \in G_{m}, m \geq 1$, the 
series

$$
\log \left(\rho^{\theta}(g)\right)=\sum_{k \geq 1} \frac{(-1)^{k+1}}{k}\left(\rho^{\theta}(g)-\mathrm{id}\right)^{k} \in \operatorname{End}_{\mathbb{Q}}\left(\hat{U}\left(\bar{K}_{\bullet}^{\mathbb{Q}}\right)\right)
$$

converges and its restriction to $\bar{K}_{0}^{\mathbb{Q}}=K_{0} / K_{1}$ and $\bar{K}_{+}^{\mathbb{Q}}=\bar{K}_{+} \otimes \mathbb{Q}$ defines an element $\varrho^{\theta}(g)$ of the degree $\geq m$ part of $\widehat{\operatorname{Der}}_{+}\left(\bar{K}_{\bullet}^{\mathbb{Q}}\right)$.

Proof. Let $g \in G_{m}, m \geq 1$ and let $r=\rho(g)$. Since

$$
r(x)=x+\left(r(x) x^{-1}-1\right) x \in\left(x+J_{m}^{\mathbb{Q}}\left(K_{*}\right)\right) \quad \text { for } x \in K_{0},
$$

we have $(r-\mathrm{id})\left(\widehat{\mathbb{Q}\left[K_{*}\right]}\right) \subset \hat{J}_{m}^{\mathbb{Q}}\left(K_{*}\right)$; similarly, since

$r(x-1)=(x-1)+\left(r(x) x^{-1}-1\right) x \in\left((x-1)+J_{i+m}^{\mathbb{Q}}\left(K_{*}\right)\right) \quad$ for $x \in K_{i}, i \geq 1$, we have $(r-\mathrm{id})\left(\hat{J}_{n}^{\mathbb{Q}}\left(K_{*}\right)\right) \subset \hat{J}_{n+m}^{\mathbb{Q}}\left(K_{*}\right)$ for all $n \geq 1$. Hence

$$
(r-\mathrm{id})^{p}\left(\hat{J}_{n}^{\mathbb{Q}}\left(K_{*}\right)\right) \subset \hat{J}_{n+p m}^{\mathbb{Q}}\left(K_{*}\right) \text { for all } n \geq 0, p \geq 1 \text {. }
$$

Taking $n=0$ in (12.4), we see that

$$
\log (r)=\sum_{k \geq 1} \frac{(-1)^{k+1}}{k}(r-\mathrm{id})^{k}
$$

is well defined as a linear endomorphism of $\widehat{\mathbb{Q}\left[K_{*}\right]}$ and, taking $p=1$ in $(12.4)$, we see that $\log (r)$ increases the filtration step by $m$ :

$$
\log (r)\left(\hat{J}_{n}^{\mathbb{Q}}\left(K_{*}\right)\right) \subset \hat{J}_{n+m}^{\mathbb{Q}}\left(K_{*}\right) \quad \text { for all } n \geq 0 .
$$

Furthermore, since $r$ is an algebra automorphism, $\log (r)$ is a derivation of the algebra $\widehat{\mathbb{Q}\left[K_{*}\right]}$. (It is well known that the logarithm of an algebra automorphism is a derivation whenever it is defined; see e.g. [33, Theorem 4], whose combinatorial argument given for a commutative algebra works in general.)

Of course, the conclusions of the previous paragraph for $r$ apply to $r^{\theta}:=\rho^{\theta}(g)$ as well. Thus we obtain

$$
\left(r^{\theta}-\mathrm{id}\right)^{p}\left(\hat{U}_{\geq n}\left(\bar{K}_{\bullet}^{\mathbb{Q}}\right)\right) \subset \hat{U}_{\geq n+p m}\left(\bar{K}_{\bullet}^{\mathbb{Q}}\right) \text { for all } n \geq 0, p \geq 1,
$$

and $\log \left(r^{\theta}\right)$ is a well-defined derivation of the algebra $\hat{U}\left(\bar{K}_{\bullet}^{\mathbb{Q}}\right)$ which increases the filtration step by $m$.

Now we prove that $\log \left(r^{\theta}\right)$ maps $\hat{U}\left(\bar{K}_{+}^{\mathbb{Q}}\right) \cdot x$ into itself for each $x \in \bar{K}_{0}$ : it suffices to prove the same property for $r^{\theta}$. As a topological vector space, $\hat{U}\left(\bar{K}_{+}^{\mathbb{Q}}\right)$ is spanned by its group-like elements: for instance, this follows from Remark 12.3 since $\widehat{\mathbb{Q}\left[K_{+}\right]}$is spanned by the homomorphic image of $K_{1}$ as a topological vector space. Therefore, it suffices to check $r^{\theta}(u \cdot x) \in \hat{U}\left(\bar{K}_{+}^{\mathbb{Q}}\right) \cdot x$ for any group-like $u \in \hat{U}\left(\bar{K}_{+}^{\mathbb{Q}}\right)$. Since $u \cdot x$ is group-like, $r^{\theta}(u \cdot x)$ is group-like and, by Lemma 11.1, we have

$$
r^{\theta}(u \cdot x)=\exp (\ell) \cdot y=y+\ell \cdot y+\frac{1}{2} \ell^{2} \cdot y+\cdots
$$

for some $\ell$ in the degree-completion $\hat{\bar{K}}_{+}^{\mathbb{Q}}$ of $\bar{K}_{+}^{\mathbb{Q}}$ and $y \in \bar{K}_{0}$. Property (12.5) with $p=1$ shows that $r^{\theta}$ induces the identity on the associated graded. Hence $r^{\theta}(u \cdot x)$ and $u \cdot x$ have the same degree 0 part, and we deduce that $y=x$.

Next, we show that $\log \left(r^{\theta}\right)$ maps any $x \in \bar{K}_{0}$ into $\hat{\bar{K}}_{+}^{\mathbb{Q}} \cdot x$. By the previous paragraph, we have $\log \left(r^{\theta}\right)(x)=t x$ for some $t \in \hat{U}\left(\bar{K}_{+}^{\mathbb{Q}}\right)$. Thus we need to show 
that $t$ is primitive. Since $r^{\theta}$ is a coalgebra homomorphism, $\log \left(r^{\theta}\right)$ is a coderivation. It follows that

$$
\begin{aligned}
\Delta(t x) & =\left(\log \left(r^{\theta}\right) \otimes \mathrm{id}+\mathrm{id} \otimes \log \left(r^{\theta}\right)\right) \Delta(x) \\
& =\left(\log \left(r^{\theta}\right) \otimes \mathrm{id}+\mathrm{id} \otimes \log \left(r^{\theta}\right)\right)(x \hat{\otimes} x)=t x \hat{\otimes} x+x \hat{\otimes} t x
\end{aligned}
$$

and we deduce that $\Delta(t)=t \hat{\otimes} 1+1 \hat{\otimes} t$. Similarly, we can show that $\log \left(r^{\theta}\right)$ maps any $\ell \in \bar{K}_{+}^{\mathbb{Q}}$ to $\hat{\bar{K}}_{+}^{\mathbb{Q}}$ : indeed, by the previous paragraph, we know that $\log \left(r^{\theta}\right)(\ell)$ belongs to $\hat{U}\left(\bar{K}_{+}^{\mathbb{Q}}\right)$ and, using that $\log \left(r^{\theta}\right)$ is a coderivation, it is easily checked that $\log \left(r^{\theta}\right)(\ell)$ is primitive.

Thus, by the previous paragraph, we can define a map $d_{0}: \bar{K}_{0} \rightarrow \hat{\bar{K}}_{+}^{\mathbb{Q}}$ and a group homomorphism $d_{+}: \bar{K}_{+}^{\mathbb{Q}} \rightarrow \hat{\bar{K}}_{+}^{\mathbb{Q}}$ by

$$
\log \left(r^{\theta}\right)(x)=d_{0}(x) \cdot x \quad \text { and } \quad \log \left(r^{\theta}\right)(\ell)=d_{+}(\ell)
$$

respectively. It remains to show that $\left(d_{0}, d_{+}\right)$is an element of $\widehat{\operatorname{Der}}_{+}\left(\bar{K}_{\bullet}^{\mathbb{Q}}\right)$, i.e., $\left(d_{0}, d_{+}\right)$is an infinite sum of derivations of the eg-Lie $\mathbb{Q}$-algebra $\bar{K}_{\bullet}^{\mathbb{Q}}$. (Those derivations will have degree $\geq m$ since we have seen that $\log \left(r^{\theta}\right)$ increases the filtration step by $m$.)

First, $d_{+}$consists of derivations (in the usual sense) of the Lie $\mathbb{Q}$-algebra $\bar{K}_{+}^{\mathbb{Q}}$ since it is a restriction of the derivation $\log \left(r^{\theta}\right)$ of the algebra $\hat{U}\left(\bar{K}_{\bullet}^{\mathbb{Q}}\right)$. Next, we check that $d_{0}$ is a 1-cocycle. For any $x, y \in \bar{K}_{0}$, we have

$$
\begin{aligned}
\log \left(r^{\theta}\right)(x y) & =x \cdot \log \left(r^{\theta}\right)(y)+\log \left(r^{\theta}\right)(x) \cdot y \\
& =x \cdot d_{0}(y) \cdot y+d_{0}(x) \cdot x \cdot y=\left({ }^{x} d_{0}(y)+d_{0}(x)\right) \cdot x y,
\end{aligned}
$$

which shows that $d_{0}(x y)=d_{0}(x)+{ }^{x} d_{0}(y)$. Finally, for any $x \in \bar{K}_{0}$ and $\ell \in \bar{K}_{+}^{\mathbb{Q}}$, we have

$$
\begin{aligned}
\log \left(r^{\theta}\right)\left({ }^{x} \ell\right) & =\log \left(r^{\theta}\right)\left(x \cdot \ell \cdot x^{-1}\right) \\
& =\log \left(r^{\theta}\right)(x) \cdot \ell \cdot x^{-1}+x \cdot \log \left(r^{\theta}\right)(\ell) \cdot x^{-1}+x \cdot \ell \cdot \log \left(r^{\theta}\right)\left(x^{-1}\right) \\
& =d_{0}(x) \cdot{ }^{x} \ell+{ }^{x} d_{+}(\ell)-x \cdot \ell \cdot x^{-1} \cdot \log \left(r^{\theta}\right)(x) \cdot x^{-1} \\
& =d_{0}(x) \cdot{ }^{x} \ell+{ }^{x} d_{+}(\ell)-{ }^{x} \ell \cdot d_{0}(x),
\end{aligned}
$$

which shows that $d_{+}\left({ }^{x} \ell\right)=\left[d_{0}(x),{ }^{x} \ell\right]+{ }^{x} d_{+}(\ell)$. We conclude that $\varrho^{\theta}(g):=\left(d_{0}, d_{+}\right)$ belongs to $\widehat{\operatorname{Der}}_{+}\left(\bar{K}_{\bullet}^{\mathbb{Q}}\right)$.

We can now prove the main result of this section.

Theorem 12.6. Let an $N$-series $G_{+}$of a group $G$ act on a formal extended $N$ series $K_{*}$ with an expansion $\theta$. Then the filtration-preserving map

$$
\varrho^{\theta}: G \longrightarrow \widehat{\operatorname{Der}}_{+}\left(\bar{K}_{\bullet}^{\mathbb{Q}}\right)
$$

in Lemma 12.5 induces the rational version of the Johnson morphism:

$$
\operatorname{gr}\left(\varrho^{\theta}\right)=\bar{\tau}_{+}^{\mathbb{Q}}: \bar{G}_{+} \longrightarrow \operatorname{Der}_{+}\left(\bar{K}_{\bullet}^{\mathbb{Q}}\right)
$$

Proof. Let $g \in G_{m}, m \geq 1$. Set $r=\rho(g)$ and $r^{\theta}=\rho^{\theta}(g)$. The leading term of $\varrho^{\theta}(g)$ is a derivation of degree $m$ of the eg-Lie $\mathbb{Q}$-algebra $\bar{K}_{\bullet}^{\mathbb{Q}}$, which is denoted by $d=\left(d_{i}\right)_{i \geq 0}$. 
We prove that $d_{0}: \bar{K}_{0} \rightarrow \bar{K}_{m} \otimes \mathbb{Q}$ is the rationalization of $\tau_{m}(g)_{0}: \bar{K}_{0} \rightarrow \bar{K}_{m}$. Let $x \in \bar{K}_{0}$. By definition of $d_{0}$, we have

$$
\log \left(r^{\theta}\right)(x) \cdot x^{-1}=d_{0}(x)+(\operatorname{deg}>m) \in \hat{\bar{K}}_{+}^{\mathbb{Q}} .
$$

Besides, it follows from (12.5) that

$$
\log \left(r^{\theta}\right)(x)=\left(r^{\theta}(x)-x\right)+(\operatorname{deg}>m) \in \hat{U}\left(\bar{K}_{\bullet}^{\mathbb{Q}}\right) ;
$$

hence

$$
d_{0}(x)=\left(\text { degree } m \text { part of }\left(r^{\theta}(x) \cdot x^{-1}-1\right)\right) .
$$

Let $y \in K_{0}$ be a representative of $x$ : since $\theta(y)=x+(\operatorname{deg} \geq 1)$ by (12.1), we have $\hat{\theta}^{-1}(x)=\iota(y) z$, where $z \in\left(1+\hat{J}_{1}^{\mathbb{Q}}\left(K_{*}\right)\right)$. Therefore,

$$
\begin{aligned}
\hat{\theta}^{-1}\left(r^{\theta}(x) \cdot x^{-1}-1\right) & =r\left(\hat{\theta}^{-1}(x)\right)\left(\hat{\theta}^{-1}(x)\right)^{-1}-1 \\
& =r(\iota(y)) r(z) z^{-1} \iota(y)^{-1}-1 .
\end{aligned}
$$

However, (12.4) shows that $r(z)-z \in \hat{J}_{m+1}^{\mathbb{Q}}\left(K_{*}\right)$, which implies that $r(z) z^{-1}$ is congruent to 1 modulo $\hat{J}_{m+1}^{\mathbb{Q}}\left(K_{*}\right)$. It follows that

$$
\hat{\theta}^{-1}\left(r^{\theta}(x) \cdot x^{-1}-1\right) \equiv r(\iota(y)) \iota(y)^{-1}-1 \quad\left(\bmod \hat{J}_{m+1}^{\mathbb{Q}}\left(K_{*}\right)\right) .
$$

We deduce that

$$
d_{0}(x)=(\text { degree } m \text { part of }(\theta([g, y])-1)) \stackrel{(12.1)}{=}\left([g, y] K_{m+1}\right)=\tau_{m}(g)_{0}(x) .
$$

Let $i \geq 1$. Now we prove that $d_{i}: \bar{K}_{i} \otimes \mathbb{Q} \rightarrow \bar{K}_{i+m} \otimes \mathbb{Q}$ is the rationalization of $\tau_{m}(g)_{i}: \bar{K}_{i} \rightarrow \bar{K}_{i+m}$. Let $\ell \in \bar{K}_{i}$. By definition of $d_{i}$, we have

$$
\log \left(r^{\theta}\right)(\ell)=d_{i}(\ell)+(\operatorname{deg}>i+m)
$$

Besides, it follows from (12.5) that

$$
\log \left(r^{\theta}\right)(\ell)=\left(r^{\theta}(\ell)-\ell\right)+(\operatorname{deg}>i+m) \in \hat{U}\left(\bar{K}_{\bullet}^{\mathbb{Q}}\right) ;
$$

hence

$$
d_{i}(\ell)=\left(\text { degree }(i+m) \text { part of }\left(r^{\theta}(\ell)-\ell\right)\right) .
$$

Let $y \in K_{i}$ be a representative of $\ell$. Then we have $\theta(y)=1+\ell+(\operatorname{deg}>i)$ by (12.1), which implies that $\hat{\theta}^{-1}(\ell) \equiv(\iota(y)-1)\left(\bmod \hat{J}_{i+1}^{\mathbb{Q}}\left(K_{*}\right)\right)$. Using $(12.4)$, we deduce that

$$
\begin{aligned}
\hat{\theta}^{-1}\left(r^{\theta}(\ell)-\ell\right)=(r-\mathrm{id})\left(\hat{\theta}^{-1}(\ell)\right) & \equiv(r-\mathrm{id})(\iota(y)-1) \quad\left(\bmod \hat{J}_{m+i+1}^{\mathbb{Q}}\left(K_{*}\right)\right) \\
& =r(\iota(y))-\iota(y) \\
& \equiv r(\iota(y))(\iota(y))^{-1}-1 \quad\left(\bmod \hat{J}_{m+i+1}^{\mathbb{Q}}\left(K_{*}\right)\right) .
\end{aligned}
$$

We conclude that

$$
d_{i}(\ell)=(\operatorname{degree}(i+m) \text { part of } \theta([g, y]-1)) \stackrel{(12.1)}{=}\left([g, y] K_{i+m+1}\right)=\tau_{m}(g)_{i}(\ell) .
$$

Remark 12.7. We can regard the map $\varrho^{\theta}: G \rightarrow \widehat{\operatorname{Der}}_{+}\left(\bar{K}_{\bullet}^{\mathbb{Q}}\right)$ in Theorem 12.6 as a "linearization" or an "infinitesimal version" of the extended N-series action of $G_{+}$ on $K_{*}$. Let $\widehat{\operatorname{Der}}_{+}\left(\bar{K}_{\bullet}^{\mathbb{Q}}\right)_{\mathrm{BCH}}$ denote the group whose underlying set is $\widehat{\operatorname{Der}}_{+}\left(\bar{K}_{\bullet}^{\mathbb{Q}}\right)$ and whose multiplication · is defined by the Baker-Campbell-Hausdorff series:

$$
d \cdot e:=d+e+\frac{1}{2}[d, e]+\frac{1}{12}[d,[d, e]]+\frac{1}{12}[e,[e, d]]+\cdots \quad \text { for } d, e \in \widehat{\operatorname{Der}}_{+}\left(\bar{K}_{\bullet}^{\mathbb{Q}}\right) .
$$


(Here $[\cdot, \cdot]$ denotes the degree-completion of the Lie bracket defined in Theorem 5.2.) Then

$$
\varrho^{\theta}: G \longrightarrow \widehat{\operatorname{Der}}_{+}\left(\bar{K}_{\bullet}^{\mathbb{Q}}\right)_{\mathrm{BCH}}
$$

is a group homomorphism, which maps $G_{+}$into the N-series of $\widehat{\operatorname{Der}}_{+}\left(\bar{K}_{\bullet}^{\mathbb{Q}}\right)_{\mathrm{BCH}}$ whose $m$ th term is $\widehat{\operatorname{Der}}_{\geq m}\left(\bar{K}_{\bullet}^{\mathbb{Q}}\right)$ for every $m \geq 1$.

Remark 12.8. In Theorem 12.6, let $K_{+}$be an $\mathrm{N}_{0}$-series of $K_{1}$ (see Section 8.2). Then the canonical map $\bar{K}_{+} \rightarrow \bar{K}_{+}^{\mathbb{Q}}$ is injective. Therefore, one can trade the Johnson morphism $\bar{\tau}_{\bullet}$ with its rational version $\bar{\tau}_{\bullet}^{\mathbb{Q}}$ without loss of information. It follows that the map $\varrho^{\theta}$ in Theorem 12.6 determines all the Johnson homomorphisms.

Example 12.9. Assume as in Example 9.5 that $K_{*}$ is the extended $\mathrm{N}$-series associated with the lower central series of $K_{0}=K_{1}:=\pi_{1}\left(\Sigma_{g, 1}, \star\right)$, and let $G_{*}$ denote the "classical" Johnson filtration of $G_{0}:=\operatorname{MCG}\left(\Sigma_{g, 1}, \partial \Sigma_{g, 1}\right)$. Then, by Proposition 8.3, $G_{+}$is an $\mathrm{N}_{0}$-series of $G:=G_{1}$, namely the Torelli group of $\Sigma_{g, 1}$. Since $K_{0}$ is a free group, Example 12.1 applies: an expansion of $K_{*}$ is a homomorphism

$$
\theta: K_{0} \longrightarrow \hat{T}\left(H^{\mathbb{Q}}\right), \quad \text { where } H^{\mathbb{Q}}=H_{1}(\Sigma ; \mathbb{Q})
$$

satisfying (12.2). According to Remark 12.8, the map $\varrho^{\theta}$ in Theorem 12.6 contains all the "classical" Johnson homomorphisms. It is shown in [22] that, for an appropriate expansion $\theta$, the map $\varrho^{\theta}$ can be identified with the "tree reduction" of the LMO functor introduced in [5].

\section{REFERENCES}

[1] S. Andreadakis, On the automorphisms of free groups and free nilpotent groups. Proc. London Math. Soc. 15 (1965) 239-268.

[2] M. Asada and M. Kaneko, On the automorphism group of some pro- $\ell$ fundamental groups. Galois representations and arithmetic algebraic geometry (Kyoto, 1985/Tokyo, 1986), 137159, Adv. Stud. Pure Math., 12, North-Holland, Amsterdam, 1987.

[3] M. Asada and H. Nakamura, On graded quotient modules of mapping class groups of surfaces. Israel J. Math. 90 (1995), no. 1-3, 93-113.

[4] D. Bar-Natan, Expansions and quadraticity for groups. In preparation.

[5] D. Cheptea, K. Habiro and G. Massuyeau, A functorial LMO invariant for Lagrangian cobordisms. Geom. Topol. 12 (2008), no. 2, 1091-1170.

[6] J. Cooper, Two mod-p Johnson filtrations. J. Topol. Anal. 7 (2015), no. 2, 309-343.

[7] J. Darné, Autour du problème d'Andreadakis. Ph.D. thesis in preparation. University of Lille 1.

[8] N. Habegger and G. Masbaum, The Kontsevich integral and Milnor's invariants. Topology 39 (2000), no. $6,1253-1289$.

[9] K. Habiro and G. Massuyeau, The Kontsevich integral for bottom tangles in handlebodies. Preprint (2017) arXiv:1702.00830.

[10] K. Habiro and G. Massuyeau, in preparation.

[11] N. Jacobson, Restricted Lie algebras of characteristic p. Trans. Amer. Math. Soc. 50 (1941), 15-25.

[12] D. L. Johnson, An abelian quotient of the mapping class group $\mathcal{I}_{g}$. Math. Ann. 249 (1980), no. 3, 225-242

[13] D. L. Johnson, A survey of the Torelli group. Low-dimensional topology (San Francisco, Calif., 1981), 165-179, Contemp. Math. 20, Amer. Math. Soc., Providence, RI, 1983..

[14] M. Kaneko, Certain automorphism groups of pro- $\ell$ fundamental groups of punctured Riemann surfaces. J. Fac. Sci. Univ. Tokyo 36 (1989), 363-372.

[15] J. P. Labute, On the descending central series of groups with a single defining relation. J. Algebra 14 (1970), 16-23.

[16] M. Lazard, Sur les groupes nilpotents et les anneaux de Lie. Ann. Sci. École Norm. Sup. 71 (1954), no. 3, 101-190. 
[17] J. Levine, Homology cylinders: an enlargement of the mapping class group. Alg. Geom. Topol. 1 (2001), 243-270.

[18] J. Levine, The Lagrangian filtration of the mapping class group and finite-type invariants of homology spheres. Math. Proc. Cambridge Philos. Soc. 141 (2006), no. 2, 303-315.

[19] E. Luft, Actions of the homeotopy group of an orientable 3-dimensional handlebody. Math. Ann. 234 (1978) 279-292.

[20] R. T. McNeill, A new filtration of the Magnus kernel of the Torelli group. Preprint (2013) arXiv: 1308.3686.

[21] G. Massuyeau, Finite-type invariants of 3-manifolds and the dimension subgroup problem. J. Lond. Math. Soc. (2) 75 (2007), no. 3, 791-811.

[22] G. Massuyeau, Infinitesimal Morita homomorphisms and the tree-level of the LMO invariant. Bull. Soc. Math. France 140 (2012), no. 1, 101-161.

[23] M. Morishita and Y. Terashima, p-Johnson homomorphisms and pro-p groups. J. Algebra 479 (2017), 102-136

[24] S. Morita, Abelian quotients of subgroups of the mapping class group of surfaces. Duke Math. J. 70 (1993), 699-726.

[25] S. Morita, A linear representation of the mapping class group of orientable surfaces and characteristic classes of surface bundles. Topology and Teichmüller spaces (Katinkulta, 1995), 159-186, World Sci. Publ., River Edge, NJ, 1996.

[26] H. Nakamura and H. Tsunogai, Some finiteness theorems on Galois centralizers in pro- $\ell$ mapping class groups. J. Reine Angew. Math. 441 (1993), 115-144.

[27] Y. Nozaki, An extension of the LMO functor. Geom. Dedicata 185 (2016), 271-306.

[28] L. Paris, Residual p properties of mapping class groups and surface groups. Trans. Amer. Math. Soc. 361 (2009), no. 5, 2487-2507.

[29] I. B. S. Passi, Group rings and their augmentation ideals. Lecture Notes in Mathematics, 715. Springer, Berlin, 1979.

[30] D. S. Passman, The algebraic structure of group rings. Pure and Applied Mathematics. Wiley-Interscience New York-London-Sydney, 1977.

[31] B. Perron, Homomorphic extensions of Johnson homomorphisms via Fox calculus. Ann. Inst. Fourier (Grenoble) 54 (2004), no. 4, 1073-1106.

[32] B. Perron, Filtration de Johnson et groupe de Torelli modulo $p$, p premier. C. R. Math. Acad. Sci. Paris 346 (2008), no. 11-12, 667-670.

[33] C. Praagman, Iterations and logarithms of formal automorphisms. Aequationes Math. 30 (1986), no. 2-3, 151-160

[34] C. Reutenauer, Free Lie algebras. London Mathematical Society Monographs. New Series, 7. Oxford Science Publications. The Clarendon Press, Oxford University Press, New York, 1993.

[35] D. G. Quillen, On the associated graded ring of a group ring. J. Algebra 10 (1968) 411-418.

[36] T. Satoh, On the Johnson homomorphisms of the mapping class groups of surfaces, Handbook of group actions. Vol. I, 373-407, Adv. Lect. Math. (ALM), 31, Int. Press, Somerville, MA, 2015.

[37] T. Satoh, A survey of the Johnson homomorphisms of the automorphism groups of free groups and related topics, Handbook of Teichmüller theory. Volume V, IRMA Lect. Math. Theor. Phys., vol. 26, Eur. Math. Soc., Zürich, 2016, pp. 167-209.

[38] J. Stallings, Homology and central series of groups. J. Algebra 2 (1965) 170-181.

[39] A. I. Suciu and H. Wang, Formality properties of finitely generated groups and Lie algebras. Preprint (2015) arXiv:1504.08294.

[40] A. Vera, Ph.D. thesis in preparation. University of Strasbourg.

[41] H. Zassenhaus, Ein Verfahren, jeder endlichen p-Gruppe einen Lie-Ring mit Charakteristik p zuzuorden. Abh. Math. Sem. Univ. Hambg. 13 (1939) 200-206.

Research Institute for Mathematical Sciences, Kyoto University, Kyoto 606-8502, JAPAN

E-mail address: habiro@kurims.kyoto-u.ac.jp

irma, Université de Strasbourg \& CNRS, 67084 Strasbourg, France

\& IMB, Université Bourgogne Franche-Comté \& CNRS, 21000 Dijon, France

E-mail address: gwenael.massuyeau@u-bourgogne.fr 